\title{
FINITE GROUPS WITH SYLOW 2-SUBGROUPS OF CLASS TWO. I
}

\author{
BY \\ ROBERT GILMAN AND DANIEL GORENSTEIN
}

\begin{abstract}
In this paper we classify finite simple groups whose Sylow 2-subgroups have nilpotency class two.
\end{abstract}

1. Introduction. In this paper we shall determine all finite simple groups with Sylow 2-subgroups of nilpotency class 2 . Specifically we shall prove

MAIN THEOREM. If $G$ is a finite group with Sylow 2-subgroups of class 2, then $G$ is isomorphic to one of the following groups: $L_{2}(q), q \equiv 7$, $9(\bmod 16), A_{7}, \operatorname{Sz}\left(2^{n}\right), U_{3}\left(2^{n}\right), L_{3}\left(2^{n}\right)$, or $P S p\left(4,2^{n}\right), n \geqslant 2$.

The proof of the theorem is carried out by contradiction. A minimal counterexample $G$ is thus a simple group whose proper subgroups have composition factors which are either isomorphic to groups listed in the Main Theorem or else have abelian Sylow 2-subgroups. Since all simple groups with abelian Sylow 2-groups have been determined in [23] and [24], it follows that every composition factor of every proper subgroup of $G$ is isomorphic to one of a specified set of known simple groups. The goal of the analysis is then to prove that $G$ itself must be isomorphic to one of the groups listed in the Main Theorem, so that no counterexample exists.

If we invoke Goldschmidt's theorem [11], and the Alperin-Brauer-Gorenstein theorem [1], we see that $G$ must also satisfy the following conditions:

(a) A Sylow 2-subgroup $S$ of $G$ does not possess a nontrivial abelian subgroup which is strongly closed in $S$ with respect to $G$; and

(b) $G$ has 2-rank at least 3.

For brevity, let us say that a simple group with Sylow 2-subgroups of class 2 is restricted provided

(1) The composition factors of every proper subgroup of $G$ are isomorphic to known simple groups with Sylow 2-subgroups of class at most 2; and

(2) $G$ satisfies conditions (a) and (b) above.

Thus our Main Theorem really follows from the classification of restricted

Presented to the Society, January 25, 1973 under the title Simple groups of class two; received by the editors December $21,1973$.

AMS(MOS) subject classifications (1970). Primary 20D05. 
simple groups with Sylow 2-subgroups of class 2. We carry out this classification in two stages:

THEOREM A. If $G$ is a restricted simple group with Sylow 2-subgroups of class 2, then every 2-local subgroup of $G$ is 2-constrained and has a trivial core.

THEOREM B. If $G$ is a restricted simple group with Sylow 2-subgroups of class 2 in which every 2-local subgroup is 2-constrained and has a trivial core, then $G \cong L_{3}\left(2^{n}\right)$ or $\operatorname{PSp}\left(4,2^{n}\right)$ for some $n \geqslant 2$.

Part I of this paper is devoted to a proof of Theorem A and Part II to a proof of Theorem B.

Theorem A is proved by contradiction. Since $G$ has Sylow 2-subgroups of class 2 and 2-rank at least 3, $\mathrm{SCN}_{3}(2)$ is nonempty in $G$. It follows therefore from a theorem of Gorenstein-Walter [19] that the centralizer of some involution of $G$ is not 2-constrained. Thus, in effect, Theorem A deals with the case of the Main Theorem in which the centralizer of some involution has a "component". The proof of Theorem $\mathbf{A}$ is divided into three major parts, as follows:

(I) The low 2-rank cases. The centralizer of every involution of $G$ is either 2-constrained or has 2-rank at most 4.

(II) The odd component case. The centralizer of some involution of $G$ has 2-rank at least 5 and possesses a component isomorphic to a group of Lie type of odd characteristic.

(III) The even component case. The components of the centralizer of every involution of $G$ are isomorphic to groups of Lie type of characteristic 2.

REMARK. For technical reasons, the group $A_{7}$ is here regarded to be of odd type, while $L_{2}(9)$ is considered to be of even type. On the other hand, the oddness or evenness of components isomorphic to $L_{2}(5)\left(\cong L_{2}(4)\right)$ is made a function of their embedding in the centralizers of the involutions of $G$.

The analysis in Case (I) (§5) is primarily fusion-theoretic and rests ultimately on the fact that a Sylow 2-subgroup $S$ of $G$ does not possess a nontrivial abelian subgroup which is strongly closed in $S$ with respect to $G$.

On the other hand, the analysis of Cases (II) and (III) $(\S \S 6,7$ for case II and $\S \S 8,9$ for Case III) consists of an elaborate application of the so-called signalizer functor method, which has at its base the Gorenstein-Goldschmidt signalizer functor theorem [14], [9], [10]. In Case (II), we are able to prove that $G$ is 3-balanced in the sense of [15] and [20], so one must work with elementary abelian 2-subgroups of $G$ of rank at least 5 to be able to assert, in general, that $A$-signalizer functors exist on $G$. Moreover, for effective application of the signalizer functor method, we need to be able to choose $A$ so that the centralizer of some involution of $\boldsymbol{A}$ has a component of odd type. Furthermore, even in Case (III), where we can establish 1 -balance, it is very convenient to be able to choose 
$A$ of rank at least 5 such that the centralizer of some involution of $\boldsymbol{A}$ is not 2-constrained. It is for these reasons that Case (I) has to be treated separately in the signalizer functor approach.

We should point out that it is very likely that a shorter and more unified proof of Theorem A-perhaps considerably shorter-could have been given if we had chosen to follow the Bender method instead. Although that method makes use of some form of Glauberman's $Z J$-theorem, which is valid only if the appropriate subgroups of $G$ are suitably $p$-stable, $p$ an odd prime, we could have established the required $p$-stability results in the present problems. Indeed, the only components which present any difficulty here are the groups $S L\left(2, p^{m}\right)$, which are not $p$-stable. However, a very simple fusion argument ( $\S 4)$ allows us, at the very outset, to exclude these groups from the list of possible components in the centralizer of any involution of $G$. It was our hope that in following the signalizer functor approach here, we would acquire a deeper understanding of how to apply it in the non 2-constrained cases of more general classification problems concerning simple groups.

Ultimately the proof of Theorem A rests upon very detailed properties of the known simple groups with Sylow 2-subgroups of class 2. These properties together with an outline of all the general machinery of the signalizer functor method is given in $\S 2$. This section, which is very long, includes as well a variety of other preliminary results which we need and, in particular, a formulation of Goldschmidt's refinement [8] of Alperin's fusion theorem for groups with class 2 Sylow 2-subgroups.

Finally completing our description of the contents of Part I, $\S 3$ gives a few additional general properties of a group $G$ which satisfies the assumptions of Theorem A.

2. Preliminary results. In this very long section we collect all the general and specialized results of a preliminary nature that we shall need for the paper.

Notation. We first describe some notation we shall use, most of which is standard.

$S(G)$ is the set of Sylow 2-subgroups of the group $G$;

$I(G)$ is the set of involutions of $G$;

$E(G)$ is the set of elementary abelian 2-subgroups of $G$;

$E_{r}(G)$ is the set of elements of $E(G)$ of 2-rank $r$;

$m(G)$ is the 2-rank of $G$-i.e. the maximum rank of an element of $E(G)$;

$M(G)$ is the set of elements of $E(G)$ of maximal rank;

$N(G)$ is the set of elements of $E(G)$ maximal under inclusion; (clearly $M(G) \subseteq N(G))$

$\bar{E}_{2^{n}}$ denotes an elementary abelian group of order $2^{n}$; 
$Z_{m}$ denotes cyclic group of order $m$;

$D_{8}$ denotes a dihedral group of order 8;

$Q_{8}$ denotes a quaternion group of order 8;

$S_{m}$ denotes a symmetric group of degree $m$.

In addition, we adopt the following convention: If $X, Y$ are subgroups of $G$ with $X \triangleleft Y$ and $D$ is a subset of $G$ which normalizes both $X$ and $Y$, then $C_{Y / X}(D)$ will denote the centralizer in $Y / X$ of the image of $D$ in $\operatorname{Aut}(Y / X)$. Likewise we shall regard the elements of $D$ as determining automorphisms of $Y / X$.

We also use the so-called "bar" convention for homomorphic images.

2-groups of class 2. The following result of Goldschmidt [7], although not actually essential for the paper, is nevertheless very useful and will provide some technical simplifications of both our notation and argument.

(2.1) If $G$ is a simple group with Sylow 2-subgroup $S$ of class 2, then $Z(S)$ is elementary.

Hence in proving our Main Theorem, we can restrict ourselves to the case in which a Sylow 2-subgroup of $G$ has an elementary center. As a result we shall only require properties of 2-groups which satisfy this condition.

Let then $S$ be a 2-group of class 2 with $Z(S)$ elementary. The following facts are trivial to verify:

(2.2) $\Phi(S)=\left\langle S^{\prime}, \mho^{1}(S)\right\rangle \subseteq Z(S)$ and every subgroup of $S$ containing $Z(S)$ is normal in $S$.

If $A \in N(S)$, then $A \supseteq \Omega_{1}(Z(S))=Z(S)$ and so we have, in particular:

(2.3) If $A \in N(G)$, then $A \triangleleft S$. Moreover, if $m(S)=r$, then $S C N_{r}(S)$ is nonempty.

If $T$ is a subgroup of $S$, then clearly $Z(T)$ need not be elementary, but necessarily $Z(T)$ contains $T^{\prime}$ and $\mho^{1}(T)$. Furthermore, we have:

(2.4) If $T$ is a subgroup of $S$ such that $C_{S}(T) \subseteq T$, then $T \triangleleft S$ and $S / \Omega_{1}(Z(T))$ is elementary abelian.

Proof. Our assumption implies that $Z(S) \subseteq T$, whence $T \triangleleft S$ and $Z(S) \subseteq \Omega_{1}(Z(T))$. But then also $S^{\prime}$ and $\mho^{1}(S)$ lie in $\Omega_{1}(Z(T))$ and consequently $S / \Omega_{1}(Z(T))$ is elementary abelian, as asserted.

Next we prove

(2.5) If $T$ is an extra-special normal subgroup of $S$, then

$$
S=T C_{S}(T) .
$$

Proof. Setting $\bar{S}=S / Z(T), \bar{S}$ must centralize $\bar{T}$ as $S$ has class 2 . The result now follows from $[12,5.4 .6]$.

Finally we recall from [15] that two elements $A, B$ of $E(S)$ of rank at 
least $k$ are said to be $k$-connected if there exists a sequence of elements of $E(S)$ of rank at least $k$

$$
A=A_{1}, A_{2}, \cdots, A_{n}=B
$$

such that, for $1 \leqslant i \leqslant n-1$, either

$$
A_{i} \subseteq A_{i+1} \text { or } A_{i+1} \subseteq A_{i} .
$$

Moreover, $S$ is said to be $k$-connected if each such pair $A, B$ is $k$-connected.

We shall need

(2.6) If $m(S) \geqslant 5$, then $S$ is 3-connected.

Proof. (Here $S$ is, of course, our 2-group of class 2.) Clearly 3-connectedness defines an equivalence relation among pairs of elements of $E(S)$ of rank at least 3. From this we easily see that it suffices to treat the case in which $A \in M(S)$ and $B \in N(S)$. In particular, $m(A)=m(S) \geqslant 5$ and $A, B$ are normal in $S$.

Let $m(A \cap B)=r$; as $\Omega_{1}(Z(S)) \subseteq A \cap B$, we may assume $r=1$ or 2. Since $A$ and $B$ are normal in $S, b \in B-A$ acts on $A$ and centralizes $A /(A \cap B)$. It follows that $|A \cap B| \geqslant|[A, b]| \geqslant\left|A: C_{A}(b)\right|$. If $r=1$, then $\left|A: C_{A}(b)\right| \leqslant 2$ and for any two involutions $b_{1}, b_{2} \in B-A,\left|A: C_{A}\left(\left\langle b_{1}, b_{2}\right\rangle\right)\right|$ $\leqslant 4$. The sequence $A_{1}=A, A_{2}=\left\langle b_{1}, b_{2}, C_{A}\left(\left\langle b_{1}, b_{2}\right\rangle\right)\right\rangle$, and $A_{3}=B$ has the required properties. When $r=2$, it suffices to pick $b \in B-A$ and change $A_{2}$ to $\left\langle b, C_{A}(b)\right\rangle$.

Components. We recall from [15] and [20] that a quasisimple group is a perfect central extension of a simple group and a (quasi)semisimple group is a central product of quasisimple groups. Moreover, any group $\boldsymbol{H}$ possesses a unique maximal normal semisimple subgroup which we denote by $L(H)$. In addition, $H$ possesses a unique normal subgroup which is minimal subject to covering $L(H / O(H))$. We denote it by $L_{2}(H)$. In the special case that $H=C_{G}(x)$, $x \in I(G)$, we shall write $L_{x}$ for $L_{2}^{\prime}(H)$.

The following result is $[18$, Theorem 1$]$ : $\mathrm{Z}\left(\mathrm{O}_{2}(\mathrm{H})\right)$.

(2.8) If $H$ is a group with $O(H)=1$, then $C_{H}\left(L(H) O_{2}(H)\right)=$

In particular, this implies

(2.9) An arbitrary group $H$ is 2-constrained if and only if $L_{2}(H)=1$.

We also have [20, Proposition 2.9]:

(2.10) The following three statements are equivalent:

(i) $L_{2^{\prime}}(H)$ is semisimple;

(ii) $L_{2}(H)=L(H)$;

(iii) $L_{2}(H)$ centralizes $O(H)$.

Moreover, we have, more generally: 
(iv) A perfect subgroup of $L_{2}(H)$ lies in $L(H)$ if and only if it centralizes $O(H)$.

The quasisimple factors of $L(H)$ are uniquely determined by $L(H)$. When $O(H)=1$, these are called the components of $H$. More generally, the uniquely determined normal subgroups of $L_{2^{\prime}}(H)$ which are minimal subject to covering the components of $L(H / O(H))$ are called the 2-components or simply the components of $H$. Clearly $H$ and $L_{2}(H)$ have the same components.

If $J$ is a component of $H$, then $J / O(J)$ is quasisimple. We shall say that $J$ is of type $K$ if $J / O(J)$ is isomorphic to the quasisimple group $K$.

For any group $G$, we denote by $L(G)$ the set of quasisimple components of $L\left(C_{G}(x) / O\left(C_{G}(x)\right)\right)$ as $x$ ranges over the involutions of $G$.

In connection with (2.10), [18, Lemma 1] is useful and yields:

(2.11) If the perfect group $K$ acts on the group $D$ of odd order, then

$$
[D, K]=[D, K, K] \text {. }
$$

We shall also need [19, Lemma 2.1] and [20, Lemma 7.4]:

(2.12) If $K$ is a semisimple group with two components $K_{1}, K_{2}$ which are interchanged by the involution $x$ which acts on $K$, then

(i) $Y=L\left(C_{K}(x)\right)=\left\{u u^{x} \mid u \in K_{1}\right\}$;

(ii) $Z(Y) \subseteq Z(K)$ and $Y$ is a homomorphic image of $K_{1}$;

(iii) If the four group $B$ acts faithfully on $K$ and contains $x$, then

$$
\left.K=\left\langle L\left(C_{K}(b)\right)\right| b \in B^{\#} \text { and } b \text { interchanges } K_{1}, K_{2}\right\rangle \text {. }
$$

Finally the following result will be important for us

(2.13) If $H$ is a group with Sylow 2-subgroup $T$ of class at most 2 and $K$ is a component of $H$, then $\left|T: N_{T}(K)\right| \leqslant 2$. Furthermore, if $K$ has nonabelian Sylow 2-subgroups, then $T$ normalizes $K$.

Proof. Without loss we can assume that $O(H)=1$. We have that $R=$ $T \cap K$ is a Sylow 2-subgroup of $K$. Set $T_{1}=N_{T}(K)$ and suppose that $T_{1} \subset$ T. Choose $t$ in $T-T_{1}$ with $t^{2} \in T_{1}$. Then $K^{t}$ is a component of $H$ distinct from $K$ and $t$ interchanges $K$ and $K^{t}$. It follows that $X=[R, t]$ $\subseteq K K^{t}$, but $X$ does not lie in $K, K^{t}$, or $Z\left(K K^{t}\right)$. On the other hand, $X \subseteq T^{\prime}$ $\subseteq Z(T)$ as $T$ has class at most 2 , so $T$ centralizes $X$. Since $T$ induces a group of permutations of the components of $H$, we conclude at once now from the embedding of $X$ in $K K^{t}$ that $T$ must leave $K K^{t}$ invariant. This immediately yields that $\left|T: T_{1}\right| \leqslant 2$.

Observe next $R$ centralizes $R^{t}$ as $K$ centralizes $K^{t}$. Hence for $y$ in $R, R$ centralizes $y^{t}$. On the other hand, $y^{-1} y^{t}=[y, t] \in Z(T)$, so $R$ centralizes $y^{-1}$ for all $y$ in $R$. Thus $y \in Z(R)$ and so $R$ is necessarily abelian when $T_{1} \subset T$. 
$C_{2}$-groups. We shall now describe groups with Sylow 2-subgroups of class at most 2 whose composition factors are of known type. To this end, we set

$$
\begin{aligned}
& A_{1}=\left\{L_{2}(q), q \text { odd, } q \neq \pm 1(\bmod 16), q>3, q \neq 9, A_{7}, J_{1},\right. \\
& \quad \text { groups of Ree type of characteristic (a power of) } 3\} ; \\
& \begin{aligned}
A_{2}=\left\{L_{2}(9), L_{2}\left(2^{n}\right), n \geqslant 3, S z\left(2^{n}\right), n\right. \text { odd, } \\
\left.n \geqslant 3, L_{3}\left(2^{n}\right), U_{3}\left(2^{n}\right), P S p\left(4,2^{n}\right), n \geqslant 2\right\} . \\
A=A_{1} \cup A_{2} .
\end{aligned}
\end{aligned}
$$

Here $J_{1}$ is Janko's first group of order 175, 560; groups of Ree type of characteristic 3 are defined and described in [23]. The groups listed in A include all simple groups with abelian Sylow 2-subgroups plus all known simple groups with Sylow 2-subgroups of class 2.

We note the additional well-known isomorphisms $A_{5} \cong L_{2}(5) \cong L_{2}(4)$, $L_{3}(2) \cong L_{2}(7)$, and $P S p(4,2) \cong S_{6}$. Thus $L_{2}(4)$ and $L_{3}(2)$ are of odd type while $P S p(4,2)$ is not quasisimple. Since $U_{3}(2)$ and $S z(2)$ are solvable groups, this will explain the bounds for $n$ in the definition of $A_{2}$.

The members of $A_{1}$ are said to be of odd type, while those of $A_{2}$ are said to be of even type.

For technical reasons, it is preferable to regard the group $L_{2}(9)\left(\cong A_{6}\right)$ as of even type rather than odd type.

More generally a quasisimple group $K$ will be called of odd or even type if $K / Z(K)$ is an element of $A_{1}$ or $A_{2}$ respectively; and a component $J$ of a group $H$ will be called of odd or even type if $J / O(J)$ is of odd or even type respectively.

Actually components $J$ of type $L_{2}(5)$ cause us considerable technical difficulties; and although at the outset we must regard them to be of odd type, later in the analysis we shall be able to consider them to be of even type. This ambiguity arises from the following possibilities:

If $J$ is a component of the group $H$ of type $L_{2}(5)$ and we set $\bar{H}=$ $H / O(H)$, then $N_{\bar{H}}(\bar{J}) / C_{\bar{H}}(\bar{J})$ is isomorphic to either $L_{2}(4)$ or $\operatorname{PGL}(2,5)$.

In the first case the balance properties of $J$ as a subgroup of $H$ are the same as those of components of even type, whereas in the second case they are analogous to components of odd type. We shall formalize this distinction in a moment.

We now define a group $H$ to be a $C_{2}$-group provided the following conditions hold:

(i) A Sylow 2-subgroup of $H$ has class at most 2; 
(ii) The nonabelian composition factors of $H$ are all isomorphic to elements of $A$.

In the body of the paper we shall be studying a simple group $G$ whose proper subgroups are all $C_{2}$-groups. For such a group, the centralizers of involutions are $C_{2}$-groups and so the elements of $L(G)$ will either be of odd or even type. We denote by $L_{1}(G)$ and $L_{2}(G)$ the corresponding subsets of $L(G)$.

Because of the ambiguity associated with $L_{2}(5)$, it will be convenient to say, if $L_{2}(5)$ is an element of $L(G)$, that $L_{2}(5)$ is evenly embedded in $L(G)$ (and in $L_{2}(G)$ ) if for any involution $x$ of $G$ and any component $J$ of $H=C_{G}(x)$ of type $L_{2}(5)$, we have

$$
N_{\bar{H}}(\bar{J}) / C_{\bar{H}}(\bar{J}) \cong L_{2}(5),
$$

where $\bar{H}=H / O(H)$.

When this is the case, we can if we wish regard $L_{2}(5)$ as an element of $L_{2}(G)$ rather than of $L_{1}(G)$, and the property of 1-balance for all components of even type will still be preserved.

To describe all possible elements of $L(G)$, we must list the possible perfect central extensions of the elements of $A$ by abelian 2-groups. All perfect central extensions of these groups are known [21], and we can easily determine those with Sylow 2-subgroups of class 2. Thus we have

(2.14) If $K$ is a quasisimple $C_{2}$-group with $O(K)=1$, then either $K$ is simple or

(i) $K \cong S L(2, q), q \equiv 3,5(\bmod 8), q>3$; or

(ii) $K / Z(K) \cong L_{3}(4)$ and $Z(K) \cong E_{2}$ or $E_{4}$.

We denote by $\hat{A}_{1}, \hat{A}_{2}$ respectively the union of $A_{1}$ with the groups listed in (i) and of $A_{2}$ with the groups listed in (ii); and we set $\hat{A}=\hat{A}_{1} \cup \hat{A}_{2}$. Then every element of $L(G)$ is an element of $\hat{A}$ and every element of $L_{1}(G)$, $L_{2}(G)$ is an element of $\hat{A}_{1}, \hat{A}_{2}$ respectively.

If $A \in E(G)$, it will be convenient also to introduce the terms $L(G ; A)$, $L_{1}(G ; A)$, and $L_{2}(G ; A)$ to denote the subsets of $L(G), L_{1}(G)$, and $L_{2}(G)$ respectively which arise from the components of $L_{x}=L\left(C_{G}(x)\right)$ of the corresponding types when $x$ is restricted to $A^{\#}$. Likewise we shall say that $L_{2}(5)$ is evenly embedded in $L_{1}(G ; A)$ if $(*)$ holds when $x$ is restricted to $A^{\#}$.

We conclude with two results related to the groups listed in (2.14). First of all, we obtain at once from (2.14) (i)

(2.15) If $\mathrm{H}$ is a $\mathrm{C}_{2}$-group such that $\mathrm{H}_{\mathrm{O}} \mathrm{O}_{2}(H) \cong L_{2}\left(2^{n}\right), n \geqslant 2$, and $\mathrm{C}_{H}\left(\mathrm{O}_{2}(H)\right) \nsubseteq \mathrm{O}_{2}(H)$, then $\mathrm{H}=\mathrm{KO}_{2}(H)$, where $K$ centralizes $\mathrm{O}_{2}(H)$ and either $K \cong L_{2}\left(2^{n}\right)$ or $n=2$ and $K \cong S L(2,5)$.

We also have 
(2.16) If $K$ is quasisimple with $O(K)=1$ and $K / Z(K) \cong L_{3}(4)$, then $E(K)$ maps onto $E(K / Z(K))$. In particular, $m(K)=4+m(Z(K))$.

Proof. The second assertion follows from the first together with the fact that $m\left(L_{3}(4)\right)=4$. Set $Z=Z(K)$ and choose $A$ in $K$ containing $Z$ so that $A / Z \in N(K / Z)$. To prove the first assertion, it will suffice to show that $A \in E(K)$. Clearly we can suppose $Z \neq 1$.

Assume first that $|Z|=2$. If $A$ is of exponent 2, then $A$ is elementary and so $A \in E(K)$. Hence we can assume that $A-Z$ contains an element $a$ of order 4. Since $K / Z \cong L_{3}(4)$, which has only one conjugacy class of involutions, this implies that every element of $A-Z$ is of order 4 . Hence $A$ possesses a unique involution and so $A \cong Q_{8}$ or $Z_{4}$. But then $A / Z \cong E_{2}$ or $E_{4}$ contrary to the fact that every element of $N(K / Z) \cong E_{16}$.

On the other hand, if $Z \cong E_{4}$, then $A / Y$ is elementary for every $Y$ in $Z$ of order 2 by the preceding argument. Clearly $A$ must then be elementary, so $A \in E(K)$ in this case as well.

Quasisimple $C_{2}$-groups; automorphisms and subgroup structure. In this subsection we list some well-known properties of a quasisimple $C_{2}$-group $K$ related to its automorphisms and subgroup structure. We consider a $C_{2}$-group $H$ containing $K$ as a normal subgroup and such that $C_{H}(K) \subseteq Z(K)$. We fix a Sylow 2-subgroup $R$ of $H$ and set $T=R \cap K$, so that $T \in S(K)$.

First of all, we have

(2.17) $H / K$ is abelian.

(2.18) One of the following holds:

(i) $R=T$;

(ii) $K \cong L_{2}(q), q \equiv 3,5(\bmod 8), O^{2^{\prime}}(H)=K R \cong P G L(2, q)$;

(iii) $K \cong L_{2}\left(q^{2}\right), q \equiv 3,5(\bmod 8)$, or $q=2^{n}, O^{2}(H)=K R$, where $R=T\langle x\rangle$ and $x$ is an involution which induces a field automorphism of $K$ of order 2 ;

(iv) $K \cong A_{7}, H \cong S_{7}$.

In particular, we have

(2.19) (i) $|R: T| \leqslant 2$;

(ii) if $K \cong L_{2}(5)$ or $L_{2}(9)$ and $H \supset K$, then $H \cong S_{5}$ or $S_{6}$ respectively;

(iii) if $K \in \hat{A}_{2}$, either $R=T$ or $K \cong L_{2}(9)$ or $L_{2}\left(2^{n}\right)$;

(iv) if $K \in \hat{A}_{2}$, then $T$ normalizes no nontrivial subgroups of $K$ of odd order and $C_{H}(T) \subseteq R$.

Suppose next that $K \cong L_{2}\left(2^{n}\right), S z\left(2^{n}\right)$, or $U_{3}\left(2^{n}\right), n \geqslant 2$, and set $A=$ $Z(T)$. Then we have

(2.20) (i) $A$ is the unique element of $M(T)=N(T)$ and $m(A)=n$;

(ii) $N_{K}(A)=T F$, where $F$ is cyclic of order $2^{n}-1,2^{n}-1$, or dividing 
$2^{2 n}-1$ respectively and $F$ acts transitively on $A^{\# \text {; }}$

(iii) $C_{H}(T)=A$;

(iv) Either $C_{H}(A) \subseteq T F$ and $C_{H}(A)=T$ or $K \cong U_{3}\left(2^{n}\right)$ and $O^{2}\left(C_{H}(A)\right)=T$;

(v) If $K \cong L_{2}\left(2^{n}\right)$ and $A_{1}$ is a subgroup of $A$ with $m\left(A_{1}\right) \geqslant n / 2$, then either $n=2$ or $A_{1}$ normalizes no nontrivial subgroups of $K$ of odd order and $\left|C_{H}\left(A_{1}\right): A\right| \leqslant 2$. Moreover, if equality holds, then $m\left(A_{1}\right)=n / 2$;

(vi) $C_{H}(F)$ is of odd order and normalizes $A$;

(vii) $C_{H}(F)=F$ unless $K \cong U_{3}\left(2^{n}\right)$.

Assume next that either $K \cong L_{3}\left(2^{n}\right)$ or $\operatorname{Psp}\left(4,2^{n}\right), n \geqslant 2$, or $K / Z(K) \cong$ $L_{3}(4)$, and put $n=2$ in the latter case. Then we have

(2.21) (i) $|M(T)|=|N(T)|=2$ and if $A \in M(T)$, then correspondingly $m(A)=2 n, m(A)=3 n$, or $m(A / Z(K))=4$;

(ii) $T$ is a special 2-group and $m(A / Z(T))=n$;

(iii) $N_{K}(A)=A(F \times J)$, where $F$ is cyclic of order dividing $2^{n}-1$ and $J \cong S L\left(2,2^{n}\right) \cong L_{2}\left(2^{n}\right)$;

(iv) If $K \cong L_{3}\left(2^{n}\right)$, then $J$ acts faithfully and irreducibly on $A$;

(v) If $K \cong P_{s p}\left(4,2^{n}\right)$, then $J$ acts indecomposably on $A, A_{0}=C_{A}(J)$ has rank $n, F$ acts transitively on $A_{0}^{\#}$, and $J$ acts faithfully and irreducibly on $A / A_{0}$;

(vi) $C_{H}(A)=A$;

(vii) If $t \in T-A$, then $C_{A}(t)=T^{\prime}=Z(T)$;

(viii) $A$ normalizes no nontrivial subgroups of $K$ of odd order.

Now assume $K \cong L_{2}(9)$ or $A_{7}$.

(2.22) If $A \in M(H)$, then $A \cap K \cong E_{4}, N_{K}(A \cap K) \cong S_{4}$, and if $K \cong$ $L_{2}(9)$, then $A=(A \cap K) \times B$, where $B$ centralizes $N_{K}(A \cap K)$.

We also have

(2.23) (i) If $K \in A_{1}$ or $K \cong L_{2}(9)$, then $R \cong E_{4}, E_{8}, D_{8}$, or $D_{8} \times E_{2}$;

(ii) If $K \in A_{1}$ or $K \cong L_{2}(9)$, then $m(R)=m(H)=2$ or 3 ;

(iii) If $K \in A_{2}$, then either $m(H)=m(K)$ or $K \cong L_{2}(9)$ and $m(H)=3$.

(iv) If $T$ is abelian, then $m(H)=m(K)$.

(2.24) If $K \in A$, then we have

(i) Either $C_{R}(T) \subseteq T$ or $R \cong D_{8} \times E_{2}$ and $K \cong L_{2}\left(q^{2}\right), q \equiv 3$, $5(\bmod 8)$ or $A_{7}$;

(ii) If $C_{R}(T) \subseteq T$, then $C_{R}(B) \subseteq T$ for each $B$ in $M(T)$.

Finally we require two results concerning the embedding of one element of $\hat{A}$ in another. For the first, we note that $P \Gamma L(2,8)$ is isomorphic to the Ree group over the field with 3 elements and so $L_{2}(8)$ is a subgroup of every Ree group of characteristic 3. (In fact, it is known that $L_{2}(8)$ is a subgroup of every group of Ree type of characteristic 3.) For the second result, we observe that $A_{6}$ 
does not contain a subgroup isomorphic to $S_{5} \cong P G L(2,5)$. Using these facts, we straightforwardly obtain:

(2.25) (i) If $K$ contains an element $L$ of $\hat{A}_{2}$ with $m(L) \geqslant 3$ and $|T: T \cap L| \leqslant 2$, then either $K=L$ or $L \cong L_{2}(8)$ and $K$ is of Ree type of characteristic 3 .

(ii) If $K \in \hat{A}_{2}$ and $K$ contains a $T$-invariant semisimple subgroup $L$ with components in $\hat{A}$, then $K=L$.

Quasisimple $C_{2}$-groups; centralizers of involutions. Again $K$ denotes an arbitrary element of $\hat{A}$ and $H, R, T$ have the same meanings as in the preceding subsection.

Let $x \in I(R)$ and set $J=L\left(C_{K}(x)\right)$. Then we have

(2.26) One of the following holds:

(i) $J=1, K \in A_{1}$, and if $K \nRightarrow A_{7}$, then $C_{H}(x)$ has a normal 2-complement and a Sylow 2-subgroup of $C_{K}(x)$ is cyclic or dihedral according as $x \in$ $R-T$ or $x \in T$;

(ii) $J=1, K \in \hat{\mathrm{A}}_{2}, O\left(C_{K}(x)\right)=1$, and either $C_{K}(x)$ is 2-closed or $K \cong$ $\operatorname{PSp}\left(4,2^{n}\right)$ or $L_{2}(9)$;

(iii) $x \in Z(K)$ and $J=K$;

(iv) $x \in T, K \cong J_{1}, C_{K}(x)=\langle x\rangle \times J$, and $J \cong L_{2}(5)$;

(v) $x \in T, K$ is of Ree type of characteristic $q=3^{m}, C_{K}(x)=\langle x\rangle \times J$, and $J \cong L_{2}(q)$;

(vi) $x \notin T, K=L_{2}\left(q^{2}\right), q \equiv 3,5(\bmod 8), C_{K}(x) \cong P G L(2, q)$, and $J \cong$ $L_{2}(q)$

(vii) $x \notin T, K \cong L_{2}\left(2^{2 n}\right), n \geqslant 2$, and $C_{K}(x)=J \cong L_{2}\left(2^{n}\right)$;

(viii) $x \notin T, K \cong A_{7}, C_{K}(x) \cong S_{5} \cong P G L(2,5)$ and $J \cong L_{2}(5)$.

These results have the following immediate consequences.

(2.27) (i) If $K \in \hat{A}_{1}$, then either $J=1$ or $J \in \hat{A}_{1}$;

(ii) If $K \in \hat{A}_{2}$, then either $J=1, J \in \hat{A}_{2}$, or $K \cong L_{2}(16)$ and $J \cong$ $L_{2}(4) \cong L_{2}(5)$;

(iii) If $K \in \hat{A}_{2}$, then $O\left(C_{K}(x)\right)=1$;

(iv) If $K \in \hat{A}_{2}$ and $x \notin Z(K)$, then $m(J)<m(K)$;

(v) $J \not L_{2}$ (9) and if $x \notin Z(K)$, then $J \neq S L(2, q), q$ odd.

It is easy to see that when $J \neq 1$, no element of $H-K$ of odd order centralizes $J$. Hence we also have

(2.28) If $J \neq 1$, then $O\left(C_{H}(x)\right)=1$ and either $\left|C_{H}(J)\right|=2$ or $J=K$ is the covering group of $L_{3}(4)$ by $E_{4}$.

We shall also need

(2.29) Let $X$ be a four subgroup of $R$. Then we have

(i) If $\bigcap_{x \in X} \#\left(C_{K}(x)\right) \neq 1$, then $K \neq A_{7}$ and $X \subseteq T$;

(ii) If $\bigcap_{x \in X} O\left(C_{K}(x)\right) \neq 1$, then $K \cong A_{7}$ or $L_{2}(q), q$ odd, and either 
$X \subseteq K$ or $K X \cong P G L(2, q)$;

(iii) If $K \cong A_{7}$, then $C_{K}(X)$ has a normal 2-complement;

(iv) If $K \cong L_{2}(q), q$ odd, and for some $y$ in $X^{\#}$, we have $K\langle y\rangle \cong$ $P G L(2, q)$, then $L\left(C_{K}(x)\right)=1$ for $x$ in $X^{\#}$;

(v) If $K \cong L_{2}(q)$ and $L\left(C_{K}(x)\right)=1$ for all $x$ in $X^{\#}$, then $C_{K}(X)$ is a 2-group.

(2.30) If $K \cong L_{2}(q), q$ odd, or $A_{7}$ and $X$ is an elementary subgroup of $R$ of order 8 , then $L\left(C_{K}(x)\right) \neq 1$ for some $x$ in $X^{\#}$. Furthermore, if $X \cap K$ centralizes a subgroup of $K$ of order 3 (in which case $K \cong A_{7}$ ), then $X \cap K \subseteq L\left(C_{K}(x)\right)$.

Finally we can describe the conjugacy classes of involutions of $K$.

(2.31) (i) $Z(T)$ contains a representative of every conjugacy class of involutions of $K$;

(ii) Either $K$ has only one conjugacy class of involutions or $K \cong$ $P s p\left(4,2^{n}\right)$ or $Z(K) \neq 1$ and $K / Z(K) \cong L_{3}(4)$;

(iii) Every involution of $T$ lies in an element of $M(T)$.

Quasisimple $C_{2}$-groups; balance and generation. The signalizer functor method depends ultimately upon various balance and generational properties of the elements of $\mathrm{A}$. We collect them here. Again $K, H, R$, and $T$ have the same meanings as before.

Using the structure of the centralizers of involutions in the groups $K$ and $H$, described above, one easily obtains

(2.32) (i) If $K \in \hat{A}_{1}$, then for any $B$ in $E_{3}(H)$, we have $\bigcap_{b \in B^{\#}} O\left(C_{H}(b)\right)=1$;

(ii) If $K \cong J_{1}$ or $K=H \cong L_{2}(5)$, then for any $x$ in $I(H)$, we have $O\left(C_{H}(x)\right)=1$;

(iii) If $K \in \hat{A}_{2}$, then for any $x$ in $I(H)$, we have $O\left(C_{H}(x)\right)=1$.

Thus the elements of $\hat{A}_{1}$ are, in general, (locally) "3-balanced", while those of $\hat{A}_{2}$ are (locally) "1-balanced".

However, we note that (iii) would be false without the restriction that $H$ be a $C_{2}$-group. Indeed, if $K \cong L_{3}(4)$, Aut $(K)$ possesses an involution $x$ such that $C_{K}(x)$ is isomorphic to an extension of $Z_{3} \times Z_{3}$ by $Q_{8}$. Hence if $H=$ $K\langle x\rangle$, then $O\left(C_{H}(x)\right) \neq 1$. However, a Sylow 2-subgroup of this group $H$ has class exceeding 2 .

Likewise (ii) would be false if $K \cong L_{2}(5)$ and $H \cong P G L(2,5)$, as then $C_{K}(x) \cong S_{3}$ for any $x$ in $I(H-K)$ and again $O\left(C_{H}(x)\right) \neq 1$. It is precisely this fact which requires us to consider components of type $L_{2}(5)$, at the outset, as being of odd type.

We also have a slight strengthening of (iii).

(2.33) If $K \in \hat{A}_{2}$ and $x \in I(R)$ and if we set $Q=C_{T}(x)$, then $Q$ 
normalizes no nontrivial subgroups of $H$ of odd order.

We turn now to generational properties. For the elements of odd type, the principal result we need is the following:

(2.34) If $K \in \hat{A}_{1}$ and $B \cong E_{8}$ acts on $K$, then

$$
K=\left\langle L\left(C_{K}(b)\right) O\left(C_{K}(b)\right) \mid b \in B^{\#}\right\rangle .
$$

This result is easily obtained from the known structures of $C_{K}(b)$ together with the observation that if $b$ in $B^{\#}$ centralizes $K$, then $K=L\left(C_{K}(b)\right)$.

We note that (2.34) is false for $L_{2}(9)$ and it is for this reason that we have excluded it from $A_{1}$. (Actually (2.34) holds when $B$ is a four group except when $K \cong L_{2}(5), L_{2}(7)$, or $A_{7}$.)

We need a number of slight variations of (2.34).

(2.35) (i) If $K \cong J_{1}$ or $K$ is of Ree type of characteristic 3, then

$$
K=\left\langle L\left(C_{K}(t)\right) \mid t \in T^{\#}\right\rangle ;
$$

(ii) If $K \in A_{1}$, either $K \cong L_{2}(5)$ or $K=\left\langle C_{K}(t) \mid t \in I(T)\right\rangle$.

(iii) If $K$ is of Ree type of characteristic 3, then

$$
K=\left\langle O\left(C_{K}(X)\right) \mid X \in E_{2}(T)\right\rangle ;
$$

(iv) If $K \cong L_{2}(q), q$ odd, $q>9$, and $X \in E_{2}(T)$, then

$$
K=\left\langle L\left(C_{K}(x)\right) O\left(C_{K}(x)\right) \mid x \in X^{\#}\right\rangle ;
$$

(v) If $K \cong A_{7}$, then $K=\left\langle N_{K}(X) \mid X \in E_{2}(T)\right\rangle$;

(vi) If $H \cong P G L(2,5)$, then $K=\left\langle N_{K}(T), C_{K}(x)\right|$ for any $x$ in $I(R-T)\rangle$.

In addition, we have

(vii) If $K \cong L_{2}(q), q \equiv 3,5(\bmod 8)$ and $X$ is a nontrivial subgroup of $K$ of odd order which centralizes an involution of $K$, then for some $u$ in $K$;

$$
\left.\left\langle X, X^{u}\right\rangle \cong L_{2}(r), \quad \text { where } r \mid q \text { and } r\right\rangle 5 \text {; }
$$

(viii) If $H \cong S_{6}$ and $A, B$ are distinct elements of $E_{3}(R)$ or $E_{2}(T)$, then $K=\left\langle N_{K}(A), N_{K}(B)\right\rangle$;

(ix) If $H=S_{7}$ and $A, B$ are distinct elements of $E_{3}(R)$ and $t$ is an involution of $T$, then

$$
K=\left\langle N_{K}(A), N_{K}(B), C_{K}(t)\right\rangle .
$$

Now we turn to components of even type.

(2.36) (i) If $K \in \hat{A}_{2}$, and $K \not L_{2}\left(2^{n}\right), S z\left(2^{n}\right), U_{3}\left(2^{n}\right)$, then $K=$ $\left\langle C_{K}(t) \mid t \in I(T)\right\rangle$;

(ii) If $K \cong L_{2}\left(2^{n}\right), S z\left(2^{n}\right)$, or $U_{3}\left(2^{n}\right), n \geqslant 2$, and $F$ is a complement to 
$T$ in $N_{K}(T)$, and $u$ is an involution inverting $F$, then $K=\langle T, u\rangle$;

(iii) If $K \cong L_{2}(9), L_{3}\left(2^{n}\right), P S p\left(4,2^{n}\right), n \geqslant 2$, or $K / Z(K) \cong L_{3}(4)$, and if $A_{1}, A_{2}$ are the two elements of $M(T)$, then

$$
K=\left\langle N_{K}\left(A_{1}\right), N_{K}\left(A_{2}\right)\right\rangle \text {; }
$$

(iv) If $K \cong L_{2}\left(2^{2 n}\right), n \geqslant 2, R \supset T, x \in I(R-T)$, and $y$ is any involution of $C_{T}(x)$, then

$$
K=\left\langle L\left(C_{K}(x)\right), L\left(C_{K}(x y)\right)\right\rangle=\left\langle N_{K}(T), C_{K}(x)\right\rangle ;
$$

(v) If $K \cong L_{2}\left(2^{n}\right), n \geqslant 2$, and $X$ is a nontrivial 2-subgroup of $K$, then there exist elements $u, v$ in $K$ such that

$$
K=\left\langle X, X^{u}, X^{v}\right\rangle \text {. }
$$

Elementary abelian 2-subgroups of maximal rank. We shall need information concerning the way in which the elements of $M(G)$ can embed in the proper subgroups of $G$. This is contained in the following statements:

(2.37) Let $H$ be a $C_{2}$-group, let $A \in M(H)$, and let $K$ be a component of $L_{2^{\prime}}(H)$. Then we have:

(i) $A$ normalizes $K$;

(ii) $A \cap K \neq 1$;

(iii) Either $A \cap K \in M(K)$ or $K$ is of type $L_{2}(q), q \equiv 3,5(\bmod 8)$ and some element of $A$ induces a nontrivial outer automorphism of $K / O(K)$;

(iv) If $K$ is of type $L_{2}\left(2^{n}\right)$, then every element of $A$ induces an inner automorphism of $K / O(K)$.

Proof. We can assume without loss that $O(H)=1$. By (2.13), either (i) holds or $K$ has abelian Sylow 2-subgroups and the normal closure $J$ of $K$ under the action of $A$ consists of two components $K, K^{a}$ for some $a$ in $A^{\#}$. Consider the latter case and set $L=L\left(C_{J}(a)\right)$, so that $L$ is $A$-invariant and $L \cong K$. By $(2.18)$, if $m(L)=r$, then also $m(\operatorname{Aut}(L))=r$. Hence if we set $A_{1}=C_{A}(L)$, it follows that $m\left(A_{1}\right) \geqslant m(A)-r$. On the other hand, $A_{1}$ clearly contains a subgroup $A_{0}$ of index 2 leaving both $K$ and $K^{a}$ invariant. But as $L=\left\{u u^{a} \mid u \in K\right\}$ and $J=K \times K^{a}$ with $A_{0}$ centralizing $L$, it is immediate that $A_{0}$ centralizes $J$. Since $J \cap A_{0}=1$ and $m(J)=2 r$, this forces

$$
m(A) \geqslant m(H) \geqslant m\left(A_{0}\right)+2 r .
$$

However, this is impossible as $m\left(A_{0}\right)=m\left(A_{1}\right)-1 \geqslant m(A)-r-1$ and $r \geqslant 2$. Thus (i) holds.

In view of (i), we can assume without loss that $H=K A$ in proving (ii), (iii), and (iv). Choose $R$ in $S(H)$ with $A \subseteq R$ and set $T=R \cap K$, so that $T \in S(K)$ and $T \triangleleft R$. Since $A \in M(R), \Omega(Z(R)) \subseteq A$. But $T \cap Z(R) \neq 1$ 
as $T \triangleleft R$ and $T \neq 1$, so (ii) holds. Likewise (iii) follows if $K \cong S L(2, q)$. Furthermore, if $K / Z(K) \cong L_{3}(4)$, then $A$ induces only inner automorphisms of $K$ and so $R=T C_{R}(T)$. Clearly then $A \cap K \in N(T)$. But now using (2.16) together with the fact that all elements of $N\left(L_{3}(4)\right)$ are isomorphic to $E_{16}$, it follows that $A \cap K \in M(T)$.

Hence we can assume that $K$ is simple. Set $A_{0}=C_{A}(K)$, so that $A_{0} \subseteq$ $Z(H)$ and $A_{0}$ is a direct factor of $H$. Hence if we set $\bar{H}=H / A_{0}$, we have that $\bar{A}$ is in $M(\bar{H})$, and it suffices to prove our assertions for $\bar{H}$. Thus without loss we can assume that $A_{0}=1$. Since $A$ leaves $C_{H}(K)$ invariant and $C_{H}(K)$ is a 2-group, this implies that $C_{H}(K)=1$, again as $A \in M(H)$. Thus $H$ is isomorphic to a subgroup of $\operatorname{Aut}(K)$. If $A$ induces only inner automorphisms of $K$, then $H=K$ and (iii) is obvious. Hence we can assume that some $a$ in $A^{\#}$ induces a nontrivial outer automorphism of $K$.

By (2.18), $K \cong L_{2}(q)$ for some even or odd $q$ or $A_{7}$, whence $T \cong E_{2^{n}}$ or $D_{8}$. If $n=2$, then $K \cong L_{2}(q), q \equiv 3,5(\bmod 8)$ and (iii).holds. Suppose then that $n>2$, in which case $K \cong L_{2}\left(2^{n}\right), n$ even, and $H=K\langle a\rangle$. By (2.26(vii)), $C_{K}(a) \cong L_{2}\left(2^{n / 2}\right)$ and so $m(A \cap K) \leqslant 1 / 2 n$, whence $m(A) \leqslant 1 / 2 n+1$. However, $m(K)=n$ and as $n>2$, we have $m(K)>m(A)$, contrary to $A \in$ $M(H)$. Thus (iii) holds in this case and also (iv) is proved.

Finally if $T \cong D_{8}$, then $R \cong D_{8} \times E_{2}$ by (2.23(i)) and it is immediate now that $A \cap K \cong E_{4}$, so (iii) holds in this case as well.

$k$-balance. As in [15] and [20], for any group $X$ and any $E$ in $E(X)$, we set

$$
\Delta_{X}(E)=\bigcap_{x \in E^{\#}} O\left(C_{X}(x)\right) .
$$

In the present context, we shall use the following modification of the standard notation of $k$-balance for quasisimple groups:

We shall say that an element $K$ of $\hat{A}$ is $k$-balanced as a $C_{2}$-group provided whenever $K$ is normal in a $C_{2}$-group $H$ with $C_{H}(K) \subseteq K$ and $E \in$ $E_{k}(H)$; we have

$$
\Delta_{H}(E)=1 .
$$

If we set $\bar{H}=H / C_{H}(K)$, then $\bar{H}$ can be identified with a subgroup of $\operatorname{Aut}(K)$ containing $\operatorname{Inn}(K)$ and to verify the given condition in $H$, it clearly suffices to show that $\Delta_{\bar{H}}(\bar{E})=1$ for all $\bar{E}$ in $E_{k}(\bar{H})$. Hence using (2.32), we obtain at once

(2.38) (i) If $K \in \hat{\mathrm{A}}_{1}$, then $K$ is 3-balanced as a $C_{2}$-group;

(ii) If $K \in \hat{A}_{2}$ or $K \cong J_{1}$, then $K$ is 1 -balanced as a $C_{2}$-group.

Now let $G$ be a simple group with Sylow 2-groups of class 2 whose proper 
subgroups are $C_{2}$-groups. For any involution $x$ of $G$, if we set $C_{x}=C_{G}(x)$ and let $K$ be a component of $L_{x}=L_{2^{\prime}}\left(C_{x}\right)$, then $\bar{H}=\operatorname{Aut}_{C_{x}}(K)=$ $N_{C_{x}}(K) / C_{C_{x}}(K / O(K))$ is a $C_{2}$-group in which the image $\bar{K}$ of $K$ is a quasisimple normal subgroup isomorphic to $K / O(K)$ and, moreover, $C_{\bar{H}}(K) \subseteq \bar{K}$. It follows therefore from the definition of local $k$-balance in $C_{x}$ of a component $K$ of $L_{x}$, given in [20, Section 5] that $K$ will be locally $k$-balanced in $C_{x}$ whenever $\bar{K}$ is $k$-balanced as a $C_{2}$-group. Note also that if $K$ is of type $L_{2}(5)$ and $L_{2}(5)$ is evenly embedded in $L(G)$, then it is immediate from the definitions that $K$ will be locally 1 -balanced in $C_{x}$.

Hence if we combine (2.38) with [20, Theorem 5.3] and use the preceding observation concerning the even embedding of $L_{2}(5)$ in $L(G)$, we can obtain conclusions about the $k$-balance of $G$. We recall from [15] and [20] that $G$ is said to be $k$-balanced if for each $T$ in $E_{k}(G)$ and each involution $x$ of $G$ which centralizes $T$, we have

$$
\Delta_{G}(T) \cap C_{G}(x) \subseteq O\left(C_{G}(x)\right)
$$

Thus [20, Theorem 5.3] yields

(2.39) (i) $G$ is 3-balanced;

(ii) If $L_{1}(G)$ is empty or consists of an evenly embedded $L_{2}(5)$, then $G$ is 1 -balanced.

The proof of $k$-balance in [20, Theorem 5.3] is actually carried out independently for each $T$ in $E_{k}(G)$ and each $x$ in $I(G)$ which centralizes $T$ and is deduced as a consequence of [20, Theorem 5.2]. Hence the same argument easily gives the following variation of (2.39):

(2.40) If $x, y$ are two commuting involutions of $G$ and $C_{G}(x)$ has no components of odd type, then

$$
O\left(C_{G}(y)\right) \cap C_{G}(x) \subseteq O\left(C_{G}(x)\right) .
$$

$L$-balance and $\Lambda$-balance. The following general result [20, Theorem 3.1] and its corollary [20, Theorem 4.1] will be basic for the paper.

(2.41) If $X$ is a 2-subgroup of the group $G$, then

$$
L_{2^{\prime}}\left(C_{G}(X)\right) \subseteq L_{2}(G)
$$

(2.42) If $x, y$ are commuting involutions of the group $G$, then

$$
L_{2^{\prime}}\left(L_{x} \cap C_{G}(y)\right)=L_{2^{\prime}}\left(L_{y} \cap C_{G}(x)\right) .
$$

This result is known as $L$-balance. (Here by definition $L_{x}=L_{2^{\prime}}\left(C_{G}(x)\right.$ ) and $L_{y}=L_{2},\left(C_{G}(y)\right)$.)

Note also, that, in particular, the left side of (2.42) lies in $L_{y}$.

We shall need some sharpenings of (2.42) in the case that $G$ is a simple 
group whose proper subgroups are $C_{2}$-groups.

(2.43) If $x, y$ are commuting involutions of $G$ and if $K$ is a component of $L_{x}$ such that $J=L_{2^{\prime}}\left(C_{K}(y)\right)$ covers $K / O(K)$, then we have

(i) The normal closure $L$ of $J$ in $L_{y}$ consists of either a single component or two components interchanged by $x$;

(ii) $J$ projects onto $L\left(C_{L / O(L)}(x)\right)$;

(iii) If $\langle x, y\rangle$ lies in an element of $M(G)$ or $J$ has nonabelian Sylow 2-subgroups, then $L$ consists of a single component.

Proof. Using [20, Proposition 4.2], we obtain (i) and the fact that $J$ projects onto a component of $L\left(C_{L / O(L)}(x)\right)$. However, the latter group is quasisimple by (2.27) and so (ii) also holds. Finally (iii) follows from (i) and correspondingly (2.37(i)) or (2.13).

We also need a slight variation of (2.41) [20, Proposition 3.4].

(2.44) If $K$ is a subgroup of the group $H$ with $L_{2^{\prime}}(K)=K$ and $K$ is normalized by a Sylow 2-subgroup of $H$, then each component of $K$ lies in a component of $L_{2}(H)$.

Next if $H$ is a $C_{2}$-group, we define $\Lambda(H)$ to be the product of all components of $L_{2}(H)$ of odd type. If $H=C_{G}(x)$ for some group $G$ and some $x$ in $I(G)$, we shall write $\Lambda_{x}$ for $\Lambda(H)$.

First of all, we have

(2.45) If $H$ is a $C_{2}$-group and $x \in I(H)$, then we have

(i) $L_{2^{\prime}}\left(C_{\Lambda(H)}(x)\right)=\Lambda\left(C_{\Lambda(H)}(x)\right)$;

(ii) $\Lambda\left(C_{L_{2}(H)}(x)\right) \subseteq \Lambda(H) J$, where $J$ is the product of those components of $L_{2^{\prime}}(H)$ of type $L_{2}(16)$ on which $x$ induces a field automorphism of order 2 .

Proof. Without loss, we assume $O(H)=1$. Let $J_{i}, 1 \leqslant i \leqslant m$, be the minimal $x$-invariant products of components of $L(H)$, so that each $J_{i}$ consists of a single component or two components interchanged by $x$. We can suppose that $\Lambda(H)$ is the product of $J_{i}, 1 \leqslant i \leqslant n$, for some $n \leqslant m$.

We have that $L_{2}\left(C_{\Lambda(H)}(x)\right)$ is precisely the product of the groups $L_{i}=$ $L_{2}\left(C_{J_{i}}(x)\right), 1 \leqslant i \leqslant n$. For $i$ in this range, if $J_{i}$ consists of two components interchanged by $x$, then $L_{i}$ is of odd type by (2.12(ii)). If $x$ centralizes $J_{i}$, then $L_{i}=J_{i}$ is of odd type, while if $J_{i}$ consists of a single component not centralized by $x$, then $L_{i}$ is of odd type (or trivial) by (2.27(i)). Thus each $L_{i}$ is of odd type (or trivial) for $1 \leqslant i \leqslant n$ and (i) follows.

Similarly $\Lambda\left(C_{L(H)}(x)\right)$ is the product of the groups $K_{i}=\Lambda\left(C_{J_{i}}(x)\right), 1 \leqslant$ $i \leqslant m$. For $i$ in the range $n+1 \leqslant i \leqslant m$, if $J_{i}$ consists of two components, then $L_{2^{\prime}}\left(C_{J_{i}}(x)\right)$ is of even type by $(2.12(i i))$ and so $K_{i}=1$. Likewise $K_{i}=1$ if $x$ centralizes $J_{i}$. On the other hand, if $J_{i}$ consists of a single component not centralized by $x$, it follows from (2.27(ii)) that either $K_{i}=1$ or $J_{i} \cong L_{2}(16)$, 
in which case $K_{i} \cong L_{2}(4) \cong L_{2}(5)$. From this (ii) follows at once.

In view of the form of (ii) above, it will be convenient to introduce the following terminology in a $C_{2}$-group $H$. If $K$ is a component of $H$ of type $L_{2}(16)$ and $x$ is an involution of $H$ which induces a field automorphism of order 2 on $K / O(K)$, we shall say simply that $x$ acts irregularly on $K$.

Using (2.45), we obtain the following variation of $L$-balance.

(2.46) If the centralizer of every involution of $G$ is a $C_{2}$-group and $x, y$ are commuting involutions of $G$, then

$$
\Lambda\left(\Lambda_{x} \cap C_{G}(y)\right) \subseteq \Lambda_{y} J_{y}
$$

where $J_{y}$ is a suitable product of components of $L_{y}$ on each of which $x$ acts irregularly and such that

$$
\Lambda\left(C_{J_{y}}(x)\right) \subseteq \Lambda_{x}
$$

Proof. Set $K=\Lambda\left(\Lambda_{x} \cap C_{G}(y)\right)$. Using $L$-balance, we see that $K$ is a product of components of $L_{2}\left(C_{L_{y}}(x)\right)$. Since the components of $K$ are of odd type, we have, in fact, $K \subseteq \Lambda\left(C_{L_{y}}(x)\right)$. Applying (2.45(ii)), we can write $K=$ $F^{*} F$, where $F^{*}$ is the product of those components of $K$ which lie in $\Lambda_{y}$ and either $F=1$ or $F$ is a product of components $F_{i}$ each of type $L_{2}(5)$ and each $F_{i} \subseteq J_{i}$ for some component $J_{i}$ of $L_{y}$ of type $L_{2}(16)$ on which $x$ acts irregularly, $1 \leqslant i \leqslant m$. In the first case, set $J_{y}=1$ and in the second, let $J_{y}$ denote the product of the $J_{i}, 1 \leqslant i \leqslant m$. Then the first inclusion of (2.46) is immediate and the second holds trivially if $F=1$. In the contrary case, $L=$ $\Lambda\left(C_{J_{y}}(x)\right)$ is the product of the groups $L_{i}=\Lambda\left(C_{J_{i}}(x)\right)$, each of which is of type $L_{2}(5)$ and contains $F_{i}$ as a normal subgroup. Since $L_{i}$ is perfect, it follows that $L_{i}=F_{i} \subseteq K$, whence $L \subseteq K$, giving the second inclusion of (2.46).

Combining (2.46) with (2.37(iv)), we obtain the following sharpening of $L$-balance, which we call $\Lambda$-balance whenever it holds.

(2.47) If $G$ is as in (2.46) and $x, y$ lie in an element of $M(G)$, then

$$
\Lambda\left(\Lambda_{x} \cap C_{G}(y)\right)=\Lambda\left(\Lambda_{y} \cap C_{G}(x)\right) .
$$

Next we can also sharpen (2.44).

(2.48) If $K$ is a subgroup of the $C_{2}$-group $H$ and $K$ is normalized by a Sylow 2-subgroup of $H$, then $\Lambda(K) \subseteq \Lambda(H)$.

PRoof. If $\bar{H}=H / O(H)$, it will clearly suffice to prove that $\Lambda(\bar{K}) \subseteq$ $\Lambda(\bar{H})$, so we can suppose without loss that $O(H)=1$. Since $\Lambda(K)$ is perfect and is invariant under a Sylow 2-subgroup of $H$, being characteristic in $K$, (2.44) implies that any component $L$ of $\Lambda(K)$ lies in a unique component $J$ of $L_{2}(H)$. We need only prove that for any such $L$, the corresponding $J$ is 
of odd type, in which case $L \subseteq J \subseteq \Lambda(H)$. Suppose then that some $J$ is of even type and let $K_{1}$ be the product of those components of $K$ which lie in $J$. Our conditions imply that $L \subseteq K_{1}$ and $K_{1}$ is invariant under a Sylow 2-subgroup $T$ of $J$. Since $O\left(K_{1}\right)$ is $T$-invariant, we have $O\left(K_{1}\right)=1$ by (2.19(iv)). Hence $K_{1}$ is semisimple and now (2.25(ii)) yields that $K_{1}=J$. Thus $K_{1}$ is quasisimple, whence $K_{1}=L$ and so $J=L$ is of odd type, which is not the case. So $L$ is also of even type, contrary to the fact that $L$ is a component of $\Lambda(K)$.

Finally we have the following variation of (2.45(i)):

(2.49) Let $H$ be a $C_{2}$-group in which $\Lambda(H) \neq 1$ and let $B \in E(H)$. Then we have

(i) If $m(B) \geqslant 3$, then $\Lambda\left(C_{\Lambda(H)}(b)\right) \neq 1$ for some $b$ in $B^{\#}$;

(ii) If $m(B) \geqslant 4$ and $\Lambda(H)$ has a component which is not of type $L_{2}(5)$, then $\Lambda\left(C_{\Lambda(H)}(b)\right)$ has a component which is not of type $L_{2}(5)$ for some $b$ in $B^{\#}$.

Proof. Without loss we can assume that $O(H)=1$. Let $K$ be a component of $\Lambda(H)$ and under the assumption of (ii), choose $K \neq L_{2}(5)$. If some $b$ in $B^{\#}$ does not leave $K$ invariant, then $K$ has abelian Sylow 2-subgroups by (2.13) and $\Lambda\left(C_{K K^{b}}(b)\right) \cong K$ by (2.12). But clearly this group is a component of $\Lambda\left(C_{\Lambda(H)}(b)\right)$ and so (i) and (ii) follow at once. Hence we can assume that $B$ normalizes $K$. If $m(B) \geqslant 4$, then some $b$ in $B^{\#}$ centralizes $K$, in which case $K$ is a component of $\Lambda\left(C_{\Lambda(H)}(b)\right)$ and again (i) and (ii) hold.

Suppose finally that $m(B) \leqslant 3$, whence equality holds and it remains only to verify (i). Likewise we can assume that $C_{B}(K)=1$, so $B$ acts faithfully on $K$. If $K \cong J_{1}$ or is of Ree type of characteristic 3 , then $\Lambda\left(C_{K}(b)\right) \neq 1$ for any $b$ in $B^{\#}$. If $K \cong A_{7}$, then some $b$ in $B^{\#}$ corresponds to a transposition in $S_{7}$ and again $\Lambda\left(C_{K}(b)\right) \neq 1$. On the other hand, if $K \cong L_{2}(q)$, then some $b$ in $B^{\#}$ must induce a field automorphism of $K$ of order 2, whence $q$ is a square. Since $q \neq 9$, it follows in this case as well that $\Lambda\left(C_{K}(b)\right) \neq 1$. Since $\Lambda\left(C_{K}(b)\right)$ is a component of $\Lambda\left(C_{\Lambda(H)}(b)\right)$, we conclude that (i) holds.

The subgroup $\Lambda^{*}(H)$. In this subsection, we introduce a certain subgroup of a $C_{2}$-group $H$, which will be very important in our analysis.

First of all, if $H$ is an arbitrary group, we define as in [20, Section 3]

$$
L_{2^{\prime}, 2}^{*}(H)=L_{2^{\prime}}(H) O(H) O\left(C_{H}(T)\right) \text {, }
$$

where $T$ is a Sylow 2-subgroup of $L_{2}(H) O_{2^{\prime}, 2}(H)$.

Then as shown in [20, Section 3], $L_{2^{\prime}, 2}^{*}(H)$ is a normal subgroup of $H$ determined independently of the choice of $T$. Moreover, $L_{2^{\prime}, 2}^{*}(H)$ contains $L_{2^{\prime}}(H)$ as a normal subgroup of odd index and each component of $L_{2^{\prime}}(H)$ is normal in $L_{2^{\prime}, 2}^{*}(H)$. 
Now assume that $H$ is a $C_{2}$-group and let $\Phi(H)$ be the product of all components of $H$ of even type, so that $L_{2}^{\prime}(H)=\Lambda(H) \Phi(H)$. We now set

$$
\Lambda^{*}(H)=O^{2}\left[C_{L_{2^{\prime}, 2}^{*}(H)}(\Phi(H) / O(\Phi(H)))\right] .
$$

(2.50) Let $T$ be a Sylow subgroup of $L_{2^{\prime}}(H) O_{2^{\prime}, 2}(H)$.

(i) $\Lambda^{*}(H)=O(H) \Lambda(H)\left[O\left(C_{H}(T)\right) \cap C_{H}(\Phi(H) / O(\Phi(H)))\right]$;

(ii) $\Lambda^{*}(H) \triangleleft H$;

(iii) $\Lambda^{*}(H)=\Lambda(H) D$, where $D$ is of odd order, $D \supseteq O(H)$, and $D$ is invariant under a Sylow 2-group of $H$;

(iv) If every component of $H$ is of odd type, then $\Lambda^{*}(H)=L_{2^{\prime}, 2}^{*}(H)$;

(v) $\Lambda^{*}\left(\Lambda^{*}(H)\right)=\Lambda^{*}(H)$.

Proof. First we prove (i). Let $F(H)$ denote the right hand side of equation (i), and let $\bar{H}=H / O(H)$. It is easy to check that $\Lambda^{*}(\bar{H})=\overline{\Lambda^{*}(H)}$ and $F(\bar{H})=\overline{F(H)}$, so we may assume $O(H)=1$. In particular $L_{2}(H)=L(H)$ and $O(\Phi(H))=1$. As $\Lambda(H)=O^{2}\left(C_{L(H)}(\Phi(H))\right.$ and $L_{2^{\prime}, 2}^{*}(H)=\Lambda(H) \Phi(H) O\left(C_{H}(T)\right)$, we have that $F(H) \subseteq \Lambda^{*}(H)$. To prove the reverse inclusion, let $x$ be an element of $\Lambda^{*}(H)$ of odd order. We can write $x=x_{1} x_{2} x_{3}$, where $x_{1} \in \Lambda(H)$, $x_{2} \in \Phi(H)$, and $x_{3} \in O\left(C_{H}(T)\right)$. In particular, $x_{1}$ and $x_{3}$ centralize $T \cap$ $\Phi(H)$. Since $x$ centralizes $\Phi(H)$, it follows that $x_{2}$ centralizes $T \cap \Phi(H)$. But as $x_{2} \in \Phi(H)$, this yields that $x_{2}$ centralizes $T$, whence $x_{2} x_{3} \in C_{H}(T)$. Thus $x \in \Lambda(H) C_{H}(T)$. But $C_{H}(T)$ has a normal 2-complement as noted in [20, Section 3]. Since $x$ has odd order, we conclude that $x \in \Lambda(H) O\left(C_{H}(T)\right)$. Thus $\Lambda^{*}(H) \subseteq \Lambda(H) O\left(C_{H}(T)\right)$. Since $\Lambda(H)$ and $\Lambda^{*}(H)$ centralize $\Phi(H)$, this in turn implies that $\Lambda^{*}(H) \subseteq F(H)$ and so (i) holds.

We see that (ii) is an immediate consequence of the definition of $\Lambda^{*}(H)$ while (iii) follows from (i). Indeed $O(H)\left[O\left(C_{H}(T)\right) \cap C_{H}(\Phi(H) / O(\Phi(H)))\right]$ is of odd order and is invariant under any Sylow 2-subgroup of $H$ which contains T. If every component of $H$ is of odd type, then $\Phi(H)=1$ and (iv) is an easy consequence of the definition of $\Lambda^{*}(H)$. Finally to prove (v) we apply (i) and (iii). Let $H^{\prime}=\Lambda^{*}(H)$; from (i) we see that $O\left(H^{\prime}\right)=O(H), \Lambda\left(H^{\prime}\right)=\Lambda(H)$ and $\Phi\left(H^{\prime}\right)=1$. Thus by (i) again $\Lambda^{*}\left(H^{\prime}\right)=O(H) \Lambda(H) O\left(C_{H^{\prime}}\left(T \cap H^{\prime}\right)\right)$. By (iii) $H^{\prime}=$ $\Lambda(H) D$. We may assume $D$ is invariant under $T \cap H^{\prime}$ whence $T \cap H^{\prime}$ centralizes $D /(D \cap \Lambda(H))$ and $H^{\prime}=\Lambda(H) C_{D}\left(T \cap H^{\prime}\right)$. By [20, Proposition 3.3] as before $C_{D}\left(T \cap H^{\prime}\right) \subseteq O\left(C_{H^{\prime}}\left(T \cap H^{\prime}\right)\right)$ and we have $H^{\prime} \subseteq \Lambda^{*}\left(H^{\prime}\right)$. Thus $H^{\prime}$ $=\Lambda^{*}\left(H^{\prime}\right)$ and $(\mathrm{v})$ holds.

We shall need the following sufficient conditions for a subgroup of $H$ of odd order to lie in $\Lambda^{*}(H)$.

(2.51) If $S \in S(H)$ and $D$ is a subgroup of $H$ of odd order, then $D$ lies in $\Lambda^{*}(H)$ under any of the following conditions: 
(i) $D$ is $S$-invariant;

(ii) For some $x$ in $I(S), D$ centralizes $x$ and is invariant under $C_{S}(x)$.

Proof. It will clearly suffice to prove the corresponding assertions for the image of $D$ in $H / O(H)$ whence we assume $O(H)=1$. By [20, Propositions 3.8 and 3.10] it follows in these cases that $D \subseteq L_{2^{\prime}, 2}^{*}(H)$ and so we need only show that $D$ centralizes $\Phi(H)$.

Let then $K$ be a component of $\Phi(H)$ and set $R=S \cap K$, so that $K$ is an element of $A_{2}$ and let $R \in S(K)$. In the case of (i), $[D, R]$ is of odd order and so $D$ leaves $K$ invariant. But then $[D, R] \subseteq K$ and so $[D, R]$ is an $R$-invariant subgroup of $K$ of odd order. Now (2.19(iv)) yields first that $D$ centralizes $R$ and then that $D$ centralizes $K$. Since $K$ was arbitrary, $D$ centralizes $\Phi(H)$ and so (i) holds.

Suppose next that we are in case (ii) and assume first that $x$ leaves $K$ invariant. Then $C_{R}(x) \nsubseteq Z(K)$ and as $\left[D, C_{R}(x)\right]$ has odd order, it follows again that $D$ leaves $K$ invariant. If $C_{R}(x)=R$, then the argument of the preceding paragraph shows that $D$ centralizes $K$, so we can assume that $x$ does not centralize $R$. If $K \cong L_{2}(9)$, then $C_{R}(x)$ is a four group. Since $\operatorname{Aut}\left(L_{2}(9)\right)$ contains no nontrivial subgroups of odd order invariant under a four group, $D$ centralizes $K$ in this case as well. Furthermore, if $K \cong L_{3}\left(2^{n}\right)$ or $P S p\left(4,2^{n}\right)$, then by $(2.18) C_{R}(x)$ contains an element of $N(R)$. But now if we apply (2.21(vi) and (viii)), we conclude, as above, that $D$ centralizes $K$. The only other possibility is that $K \cong L_{2}\left(2^{n}\right), n \geqslant 4$, and $C_{R}(x)$ is elementary of rank $1 / 2 n$; and now (2.20(v)) implies that $D$ centralizes $K$.

Assume then that $x$ does not leave $K$ invariant, whence $K \cong L_{2}\left(2^{n}\right)$ and so also $J=C_{K K^{x}}(x) \cong L_{2}\left(2^{n}\right)$. Setting $T=S \cap J$, we have that $T \in S(J)$ and, as $[D, T]$ has odd order and $D$ induces a permutation of the components of $\Phi(H)$, we see that $D$ must leave $K K^{x}$ invariant and so must leave $J$ invariant. It follows now, as above, that $D$ centralizes $J$. Hence by [20, Lemma 2.16] $D$ centralizes $K$ in this case as well. Again we conclude that $D$ centralizes $\Phi(H)$ and so (ii) also holds.

At one point in our analysis, we shall need a property of yet another subgroup related to $L_{2}(H)$. For simplicity, we restrict ourselves to the case of a $C_{2}$-group $H$ in which $O(H)=1$. We define $\widetilde{L}(H)$ to be the uniquely determined normal subgroup of $H$ which is maximal subject to the following conditions:

(a) $\widetilde{L}(H) \supseteq L(H)$;

(b) $\widetilde{L}(H) / \bar{L}(H)$ is a 2-group;

(c) $\widetilde{L}(H)$ leaves each component of $L(H)$ invariant. Clearly $\widetilde{L}(H)$ exists. 
(2.52) If $S$ is a Sylow 2-subgroup of $H$, then $Z(S) \subseteq \widetilde{L}(H)$.

Proof. Let $K$ be the subset of $H$ consisting of those elements which centralize $Q=\mathrm{O}_{2}(H)$ and leave each component of $L(H)$ invariant. Clearly $K$ is just the kernel of the permutation representation of $C_{H}(Q)$ on the components of $L(H)$. Since $C_{H}(Q)$ and $L(H)$ are each normal in $H$, it follows that $K \triangleleft$ $H$. Furthermore, $K \supseteq L(H) Z(Q)$. Since $C_{H}(L(H) Q)=Z(Q)$ by (2.8), we also have that $\bar{K}=\bar{K} / L(H) Z(Q)$ is isomorphic to a subgroup of $\operatorname{Aut}(L(H)) / \operatorname{Inn}(L(H))$. But $\operatorname{Inn}(L(H)) \cong L(H) / Z(L(H))$ and so is the direct product of simple groups $\bar{L}_{i} \in \hat{A}, 1 \leqslant i \leqslant n$. Moreover, $\bar{K}$ leaves each $\bar{L}_{i}$ invariant. We easily conclude from this that $\bar{K}$ is, in fact, isomorphic to a subgroup of the direct product of the groups $\operatorname{Aut}\left(\bar{L}_{i}\right) / \operatorname{Inn}\left(\bar{L}_{i}\right), 1 \leqslant i \leqslant n$. But now applying (2.17), we obtain that $\bar{K}$ is abelian. Since $K$ centralizes $Q$, this in turn implies that $K / L(H)$ is 2-closed. Hence if we set $N=O^{2}(K)$, we have that $N \triangleleft H, N \supseteq L(H), N / L(H)$ is a 2-group, and $N$ leaves each component of $L(H)$ invariant. We conclude therefore from the definition that $N \subseteq \widetilde{L}(H)$.

On the other hand, as $S \cap L_{i}$ is a Sylow 2-subgroup of $L_{i}$ for each component $L_{i}$ of $L(H), 1 \leqslant i \leqslant n$, and $Q \subseteq S$, we have that $Z(S)$ leaves each component of $L(H)$ invariant and centralizes $Q$, so $Z(S) \subseteq K$. Thus $Z(S) \subseteq O^{2^{\prime}}(K)=N=\widetilde{L}(H)$, as asserted.

$\Lambda^{*}$-balance and $\Lambda^{*}$-generation. We shall now establish two important properties related to components of odd type in the centralizers of involutions of a simple group $G$ whose proper subgroups are $C_{2}$-groups. For any proper subgroup $H$ of $G$, the subgroup $\Lambda^{*}(H)$ of the preceding subsection is then welldefined. In particular, $\Lambda^{*}\left(C_{G}(x)\right)$ is well-defined for any involution $x$ of $X$. For brevity, we shall set

$$
\Lambda_{x}^{*}=\Lambda^{*}\left(C_{G}(x)\right)
$$

We first prove

(2.53) Let $x, y$ be two commuting involutions of $G$. If $x$ does not act irregularly on any component of $L_{y}$ and, in particular, if $\langle x, y\rangle$ lies in an element of $M(G)$, then we have

$$
\Lambda^{*}\left(\Lambda_{x}^{*} \cap C_{G}(y)\right) \subseteq \Lambda_{y}^{*} .
$$

Proof. Set $K=\Lambda_{x}^{*} \cap C_{G}(y)$. We must prove that $\Lambda^{*}(K) \subseteq \Lambda_{y}^{*}$. Let $T$ be a Sylow 2-subgroup of $C_{G}(y)$ containing $\langle x, y\rangle$ and set $R=C_{T}(x)$, so that $R \subseteq C_{G}(x)$ and $y \in R$. By (2.50), we can write $\Lambda_{x}^{*}=\Lambda_{x} D$, where $D$ is $R$-invariant of odd order. Set $\bar{D}=\Lambda_{x} D / \Lambda_{x}$. We claim that $C_{D}(y)$ covers $C_{\bar{D}}(y)$. Indeed, we can identify $\bar{D}$ with $D / D \cap \Lambda_{x}$ and can consider the action of $y$ on $\bar{D}$ to be induced from that of $D$ under the natural map of $D$ on $D / D \cap \Lambda_{x}$. Since $D$ has odd order, our assertion follows from general proper- 
ties of $\pi^{\prime}$-automorphisms of $\pi$-groups. Hence $K=C_{\Lambda_{x} D}(y) \subseteq \Lambda_{x} C_{D}(y)$ and we conclude that $K=C_{\Lambda_{x}}(y) C_{D}(y)$.

Since $C_{D}(y)$ has odd order, the preceding equality implies that $\Lambda(K)=$ $\Lambda\left(C_{\Lambda_{x}}(y)\right)$. Now $K$ is $R$-invariant as $y \in Z(R)$ and so clearly $\Lambda^{*}(K)=$ $\Lambda^{*}(K R)$. It follows therefore from (2.50) that $\Lambda^{*}(K)=\Lambda\left(C_{\Lambda_{x}}(y)\right) E$, where $E$ is $R$-invariant of odd order. But by (2.46) and (2.47) and our hypothesis, we have that $\Lambda\left(C_{\Lambda_{x}}(y)\right) \subseteq \Lambda_{y}$. Furthermore, $E \subseteq K$ centralizes $y$ and $E$ is invariant under $R=C_{T}(y)$. Hence $E \subseteq C_{G}(y)$ and (2.51(ii)) yields that $E \subseteq$ $\Lambda_{y}^{*}$. We thus conclude that $\Lambda^{*}(K) \subseteq \overline{\Lambda_{y}^{*}}$, as required.

If $A \in M(G)$, then (2.53) holds for every pair of involutions $x, y$ of $A$. We shall say in this case that $G$ is $\Lambda^{*}$-balanced with respect to $A$.

The following basic generational result is a particular case of $[20$, Theorem 7.12] and will be called $\Lambda^{*}$-generation.

(2.54) If $H$ is a $C_{2}$-group and $B \in E_{3}(H)$, then

$$
\Lambda^{*}(H)=\left\langle\Lambda^{*}\left(C_{\Lambda^{*}(H)}(b)\right) \mid b \in B^{\#}\right\rangle .
$$

Proof. Set $X=\Lambda^{*}(H)$. Since each component of $X$ is of odd type, it follows from (2.50(iv) and (v)) that $X=L_{2^{\prime}, 2}^{*}(X)$. We shall argue that for $b$ in $B^{\#}$, we also have $\Lambda^{*}\left(C_{X}(b)\right)=L_{2^{\prime}, 2}^{*}\left(C_{X}(b)\right)$. This will follow from (2.50(iv)) provided we can show that each component of $C_{X}(b)$ is of odd type. It will clearly suffice to prove that each component of $C_{\bar{X}}(b)$ is of odd type, where $\bar{X}=X / O(X)$. Let $\bar{K}_{i}, 1 \leqslant i \leqslant n$, be the minimal $b$-invariant products of components of $L(\bar{X}), 1 \leqslant i \leqslant n$. Since $\bar{X} / L(\bar{X})$ has odd order, we see that $L_{2}\left(C_{\bar{X}}(b)\right)$ is just the product of the groups $L_{2^{\prime}}\left(C_{\bar{K}_{i}}(b)\right), 1 \leqslant i \leqslant n$. If $\bar{K}_{i}$ consists of a single component, then by $(2.27(i))$ either $L_{2^{\prime}}\left(C_{K_{i}}(b)\right)$ is trivial or consists of a component of odd type. In the contrary case, $\bar{K}_{i}$ consists of two components interchanged by $b$ and now (2.12(ii)) implies that $L_{2},\left(C_{K_{i}}{ }^{(b))}\right.$ is a nontrivial homomorphic image of one of these components and so is also of odd type. Thus each component of $C_{X}(b)$ is of odd type for each $b$ in $B^{\#}$, as asserted.

Hence (2.54) is equivalent to the following assertion:

$$
X=\left\langle L_{2^{\prime}, 2}^{*}\left(C_{X}(b)\right) \mid b \in B^{\#}\right\rangle .
$$

In the terminology of $\left[20\right.$, Section 7], this is the same as saying that $X$ is $L^{*}$. generated with respect to $B$. In view of [20, Theorem 7.12], (*) will hold if for any component $L$ of $X$ and any element $E$ of $E_{3}\left(N_{B}(L)\right)$, we have that $\bar{L}=L / O(L)$ is corelayer 1-generated with respect to $E$, which means that 


$$
\bar{L}=\left\langle L_{\mathbf{2}^{\prime}}\left(C_{\bar{L}}(y)\right) O\left(C_{\bar{L}}(y)\right) \mid y \in E^{\#}\right\rangle .
$$

However, as $\bar{L} \in \hat{A}_{1}$, this is a consequence of (2.34). Thus (*) and hence (2.54) is proved.

We need one further generational result, of a slightly difficult nature; namely, [20, Lemma 7.2].

(2.55) If $K$ is a perfect group, $B$ is an elementary abelian 2-group which centralizes $K$ and $K B$ acts on the group $D$ of odd order, then we have

$$
\left.[D, K]=\left\langle\left[C_{D}(T), K\right]\right| T \text { of index at most } 2 \text { in } B\right\rangle \text {. }
$$

Signalizer functors and $k$-balanced groups. Let $G$ be a group and $S$ a Sylow 2-subgroup of $G$.

For any positive integer $k$ and any $B$ in $E(G)$ with $m(B) \geqslant k$, we define

$$
W_{B}=\left\langle\Delta_{G}(T) \mid T \in E_{k}(B)\right\rangle,
$$

and

$$
\Gamma_{S, k}=\left\langle N_{G}(Q) \mid Q \subseteq S, m(Q) \geqslant k\right\rangle .
$$

$\Gamma_{S, k}$ is called the $k$-generated core of $G$.

If we combine [20, Theorem 6.1], which proves the existence of signalizer functors in $k$-balanced groups, with the signalizer functor theorem itself [9], [10] we obtain at once $[20$, Theorem 6.10$]$ :

(2.56) If $A \in E(G)$ with $m(A) \geqslant k+2$ and $G$ is $k$-balanced with respect to $A$, then $W_{A}$ is of odd order.

Using [20, Proposition 8.9], we also have

(2.57) If $G$ is $k$-balanced with respect to $A \in E(G)$ and $B \subseteq A$ with $m(B) \geqslant k+1$, then

(i) $W_{B}=W_{A}$; and

(ii) $N_{G}(B)$ normalizes $W_{A}$.

Likewise [20, Proposition 8.1 and Theorem 8.8] together yield

(2.58) If $G$ is $k$-balanced and $S$ is $(k+1)$-connected, then

$$
\Gamma_{S, k}(G) \subseteq N_{G}\left(W_{A, k}\right)
$$

for any $A$ in $E(S)$ with $m(A) \geqslant k+1$.

Using (2.39), (2.56) has the following corollary:

(2.59) If $S$ has class 2 and the centralizer of every involution of $G$ is a $C_{2}$-group, then $W_{A}$ is of odd order for any $A$ in $E(G)$ with $m(A) \geqslant 5$. (We take $k=3$.)

Strong embedding and balanced groups. If $G$ is a group of even order with 2-subgroup $S$, it is trivial to see that $G$ has a strongly embedded subgroup if and 
only if its 1-generated core $\Gamma_{S, 1}(G)$ is a proper subgroup of $G$.

Bender's classification theorem [3] concerning groups with a strongly embedded subgroup will be important for us.

(2.60) If $G$ is a simple group which contains a strongly embedded subgroup, then $G \cong L_{2}\left(2^{n}\right), S z\left(2^{n}\right)$, or $U_{3}\left(2^{n}\right)$ for some $n \geqslant 2$.

Aschbacher has extended Bender's theorem to the case of groups with a proper 2-generated core [2]. Although we could avoid his result in this paper, use of it makes it easier to derive a basic property of balanced (i.e. 1-balanced) groups.

(2.61) If $G$ is a simple group with $m(G) \geqslant 3$ which has a proper 2-generated core, then $G \cong J_{1}, L_{2}\left(2^{n}\right), S z\left(2^{n}\right)$, or $U_{3}\left(2^{n}\right), n \geqslant 2$.

More generally, Aschbacher's result gives a description of all quasisimple groups with a proper 2-generated core. It is then easy to check that any quasisimple group is either 2-generated or exceptional as these terms are defined in [19]. But then [2] , [19] together yield the following general assertion:

(2.62) If $G$ is a balanced, connected (i.e. 2-connected) group with $m(G) \geqslant$ 3 and $O(G)=1$, then either $O\left(C_{G}(x)\right)=1$ for every involution $x$ of $G$ or $G$ has a proper 2-generated core.

But now if we combine this with (2.61), we obtain immediately the following general theorem:

(2.63) If $G$ is a simple, balanced, connected group with $m(G) \geqslant 3$, then $O\left(C_{G}(x)\right)=1$ for every involution $x$ of $G$.

Finally we apply the preceding result to the case in which $G$ is a simple group with Sylow 2-subgroup of class 2 and 2-rank at least 3 in which the centralizer of every involution is a $C_{2}$-group.

(2.64) (i) If $L_{1}(G)$ is either empty or consists of an evenly embedded $L_{2}(5)$, then $O\left(C_{G}(x)\right)=1$ for every involution $x$ of $G$;

(ii) If $L(G)$ is empty, then $H$ is 2-constrained and $O(H)=1$ for every 2-local subgroup $H$ of $G$.

Proof. By (2.40), $G$ is balanced in either case. Furthermore, as $m(G)$ $\geqslant 3$ by assumption, $\mathrm{SCN}_{3}(2)$ is nonempty by (2.3). As is well-known (or by the argument of (2.6)), this implies that $G$ is connected. Now (i) follows at once from (2.63). Furthermore, if $L(G)$ is empty, then $C_{G}(x)$ is 2-constrained for every involution $x$ of $G$ by (2.9). But then $H$ is 2-constrained for every 2-local subgroup $H$ of $G$ by [13, Theorem 4]. Since $O\left(C_{G}(x)\right)=1$ for each involution $x$ of $G,[13$, Theorems 1 and 5] imply that also $O(H)=1$ for every 2-local subgroup $H$ of $G$.

2-constrained $C_{2}$-groups. Let $H$ be a $C_{2}$-group with $O(H)=1$ in which a Sylow 2-subgroup, $S$, has class 2 and elementary center. Set $D=O_{2}(H)$ and $Z=\Omega_{1}(Z(D))$ and define $\bar{H}=H / C_{H}(Z), \widetilde{H}=\bar{H} / O(\bar{H}), H^{*}=O^{2}{ }^{\prime}(H)$. The following general result will be used primarily in the case in which $H$ is 2-constrained. 
(2.65) (i) $\bar{S}$ is elementary abelian;

(ii) $\widetilde{H}^{*}=L(\widetilde{H}) \times O_{2}(\widetilde{H})$ and each component of $L(\widetilde{H})$ is isomorphic to $L_{2}(q), q \equiv 3,5(\bmod 8)$ or $q=2^{n}, J_{1}$, or is of Ree type of characteristic 3;

(iii) $H^{*}$ acts trivially on $D^{\prime}$ and $\mho^{1}(D)$. If $Z(S) \subseteq Z$, then $H^{*}$ centralizes $D / Z$, and $O^{2}\left(O^{2}\left(C_{H}(Z)\right)\right)$ centralizes $D$;

(iv) If $H$ is 2-constrained, then $C_{H^{*}}(Z)=D$.

(v) If $\bar{H}^{*}=\bar{H}_{1} \times \cdots \times \bar{H}_{n}$, then $\left[Z, \bar{H}_{i}, \bar{H}_{j}\right]=1$ for $i \neq j$.

Proof. By (2.2) $\mho^{1}(S) \subseteq Z(S) \subseteq C_{H}(Z)$, so $\bar{S}$ is elementary abelian. Thus (i) holds and now (ii) follows from the general classification of groups with abelian Sylow 2-subgroups [23], [24]. If $\bar{H}^{*}$ is as in (v), then for any choice of involutions $\bar{x} \in \bar{H}_{i}, \bar{y} \in \bar{H}_{j},[Z, \bar{x}, \bar{y}]=1$ because $S$ has class two. By definition of $\bar{H}^{*}$ the possible choices for $\bar{x}$ and $\bar{y}$ generate $\bar{H}_{i}$ and $\bar{H}_{j}$, and it follows that $\left[\bar{Z}, \bar{H}_{i}, \bar{H}_{j}\right]=1$.

If $Z(S) \subseteq Z$, then $S / Z$ is elementary and consequently $S$ acts trivially on $D / Z$. Since $H^{*}$ is generated by the conjugates of $S$ in $H$, it follows that $H^{*}$ acts trivially on $D / Z$. Because $D^{\prime}$ and $\mho^{1}(D)$ lie in $Z(S)$ by (2.2) and are characteristic in $D$, it follows similarly that $H^{*}$ acts trivially on each of them, so the first part of (iii) holds. Set $L=O^{2}\left(C_{H}(Z)\right)$ and $K=O^{2}(L)$. Clearly $K \subseteq L \subseteq H^{*}$ and so $K$ acts trivially on $D / Z$. But obviously $K$ acts trivially on $Z$ and as $K$ is generated by its subgroups of odd order, we conclude that $K$ centralizes $D$, proving the second part of (iii).

Finally suppose $H$ is 2-constrained, whence $Z(S) \subseteq C_{H}(D) \subseteq D$. Hence by (iii) $K$ is a 2-group and it follows that $L$ must also be a 2-group. Obviously $D \subseteq L$ and $L$ is normal in $H$, so we have $D=L$ and $\left|C_{H^{*}}(Z): D\right|$ is odd. But by (iii) elements of odd order in $C_{H^{*}}(Z)$ centralize $Z$ and $D / Z$. Thus these elements centralize $D$ and must lie in $D$. We conclude $C_{H^{*}}(Z) \subseteq D$.

If $S L\left(2,2^{n}\right)=L_{2}\left(2^{n}\right)$ acts on the elementary abelian 2-group $V$, we say that $V$ is a standard or natural module for $L_{2}\left(2^{n}\right)$ if $V$ has rank $2 n$ and can be identified with the additive group of a vector space of dimension 2 over the field $G F\left(2^{n}\right)$ in such a way that the given action of $L_{2}\left(2^{n}\right)$ becomes its natural one.

(2.66) If $H$ is 2 -constrained, $\bar{H} \cong L_{2}\left(2^{n}\right), n \geqslant 2$, and $V$ is a nontrivial $H$-composition factor of $Z$, then

(i) $m(V)=2 n$ and $V$ is a standard module for $\bar{H}$;

(ii) For any $\bar{x}$ in $\bar{S}^{\#}, C_{V}(\bar{x})=C_{V}(\bar{S})=[\bar{S}, V]=[\bar{x}, V] \cong E_{2^{n}}$;

(iii) Every element of $N_{\bar{H}}(\bar{S})^{\#}$ of odd order acts regularly on $V$;

(iv) $\left|Z(S): \mho^{1}(D)\right| \geqslant 2^{n}$ and $m(Z) \geqslant 2 n$.

Proof. Let $V$ be an arbitrary nontrivial composition factor of $Z$ under $\bar{H}$. Since $S$ has class 2 , we have $C_{V}(\bar{x}) \supseteq C_{V}(\bar{S}) \supseteq[V, \bar{S}] \supseteq[V, \bar{x}]$ and 
$\left|V: C_{V}(\bar{x})\right|=|[V, \bar{x}]|$ for any $\bar{x}$ in $\bar{S}^{\#}$. We set $a=m\left(V / C_{V}(\bar{x})\right), b=$ $m\left(C_{V}(\bar{x}) / C_{V}(\bar{S})\right), c=m\left(C_{V}(\bar{S}) /[V, \bar{S}]\right), d=m([V, \bar{S}] /[V, \bar{x}])$, and $m=$ $m(V)$. It is immediate from the subgroup structure of $L_{2}\left(2^{n}\right)$ that $\bar{H}$ is generated by $\bar{S}$ and a conjugate $\bar{y}$ of $\bar{x}$. Since $V$ is a nontrivial irreducible $\bar{H}$-module, it follows that $C_{V}(\bar{y}) \cap C_{V}(\bar{S})=1$ (as $\bar{H}$ centralizes this intersection). Likewise $\bar{S}$ and $\bar{y}$ leave invariant any subgroup of $V$ containing $[V, \bar{S}]$ and $[V, \bar{y}]$ respectively, and so it follows likewise that $V=\langle[V, \bar{S}]$, $[V, \bar{y}])$. Since $m\left(C_{V}(\bar{x})\right)=m\left(C_{V}(\bar{y})\right)$ and $m([V, \bar{x}])=m([V, \bar{y}])$, we conclude now that

$$
2 a+b \geqslant m \text { and } 2 a+d \geqslant m .
$$

But also our relations imply that $2 a+b+c+d=m$. Combined with the preceding inequalities, this forces $b=c=d=0$ and $m=2 a$. Thus the first three equalities of (ii) holds.

Now let $\bar{F}$ be a complement to $\bar{S}$ in $N_{\bar{H}}(\bar{S})$ and let $\bar{u}$ be an involution of $\bar{H}$ which inverts $\bar{F}$. Then by (2.36(ii)) we have $\bar{H}=\langle\bar{S}, \bar{u}\rangle$. Moreover we can choose $\bar{u}$ and $\bar{x} \in \bar{S}^{\#}$ so that $\langle\bar{x}, \bar{u}\rangle \cong L_{2}(2)$ and $\bar{x} \bar{u}$ has order 3 . Setting $V_{0}=C_{V}(\bar{S})$, then $\bar{u}$, being an involution, leaves $V_{0} \cap V_{0}^{\bar{u}}$ invariant and so $V_{0} \cap V_{0}^{\bar{u}}=1$. On the other hand, the results of the first paragraph imply that $m\left(V_{0}\right)=a=1 / 2 m$. Hence if we set $V_{1}=V_{0}^{\bar{u}}$, we have that $V_{0} \oplus V_{1}$.

Our conditions also imply that the mapping $v_{1} \rightarrow\left[v_{1}, \bar{x}\right]$ for $v_{1}$ in $V_{1}$ is an isomorphism of $V_{1}$ on $V_{0}$, so we can choose bases for $V_{1}$ and $V_{0}$ so that $\bar{x}$ is represented by the matrix $\left(\begin{array}{ll}I & I \\ 0 & I\end{array}\right)$, where $I$ denotes an $a \times a$ identity matrix. Since $\bar{u}$ interchanges $V_{0}$ and $V_{1}$, and $\bar{x} \bar{u}$ has order 3 , it follows that $\bar{u}$ is represented by the matrix $\left(\begin{array}{cc}0 & I \\ I_{-} & 0\end{array}\right)$. Likewise as $\bar{F}$ leaves $V_{0}$ invariant and $\bar{u}$ inverts $\bar{F}$, it follows that each $\bar{f}$ in $\bar{F}$ is represented by a matrix of the form

$$
\left(\begin{array}{cc}
A_{\bar{f}} & 0 \\
0 & A_{\bar{f}}^{-1}
\end{array}\right),
$$

where $A_{\bar{f}}$ is a suitable $a \times a$ nonsingular matrix.

Hence the matrix of $\bar{x} \bar{f}$ has the form

$$
\left(\begin{array}{cc}
I & A_{\bar{f}}^{-2} \\
0 & I
\end{array}\right)
$$

for each $\bar{f}$ in $\bar{F}$. Since the elements $\bar{x} \bar{f}$ run through $\bar{S}^{\#}$ as $\bar{f}$ runs through $\bar{F}$, it follows from the manner in which two matrices of this form multiply that the 
set of matrices $\left\{A_{\bar{f}} \mid \bar{f} \in \bar{F}\right\} \cup\{0\}$ is closed under addition. In other words, the matrices

$$
\left(\begin{array}{ll}
A_{\bar{f}} & 0 \\
0 & A_{\bar{f}}
\end{array}\right)
$$

for $\bar{f}$ in $\bar{F}$ can be identified with the multiplicative group of a field of order $2^{n}$. Since these matrices commute with those for $\bar{u}, \bar{F}$, and $\bar{S}$, we conclude that $V$ is, in fact, an irreducible $\bar{H}$-module over $G F\left(2^{n}\right)$. In particular, as $V$ must be at least 2-dimensional over $G F\left(2^{n}\right)$, we have $m \geqslant 2 n$.

Finally if $W_{0}$ is a nontrivial $\bar{F}$-submodule of $V_{0}$ and if we set $W_{1}=$ $W_{0}^{\bar{u}}$ and $W=W_{0} \oplus W_{1}$, then it follows from our matrix representation that $W$ is invariant under $\bar{H}=\langle\bar{u}, \bar{x}, \bar{F}\rangle$. Thus $W=V$ by the irreducibility of $H$ on $V$ and so $V_{0}$ is an irreducible $\bar{F}$-module, forcing $m\left(V_{0}\right)=n$. We therefore conclude that $m=2 n$, proving (i). Likewise $a=n$ and so the final assertion of (ii) also holds. Furthermore, clearly we also have (iii).

As $H$ is 2-constrained, (2.65(iv)) implies that $Z$ has a nontrivial $\bar{H}$-composition factor, $V$. By $\left(2.65\left(\right.\right.$ iii)) $\mho^{1}(D)$ must be centralized by $\bar{H}^{*}=\bar{H}$ and so $V$ is actually a composition factor of $Z / \mho^{1}(D)$. On the other hand, $Z(S) \supseteq$ $[Z, S]$ and $[Z, S]$ certainly covers $V_{0}$, so it follows that $\left|Z(S): \mho^{1}(D)\right| \geqslant$ $\left|V_{0}\right|=2^{n}$. Since $m(Z) \geqslant 2 n$ by (i), we thus obtain (iv).

(2.67) Suppose that $H$ is 2-constrained and $\bar{H}$ has a normal 2-complement. Setting $\bar{Q}=[O(\bar{H}), \bar{S}]$, we have

(i) If $|\bar{S}|=2$ and $\left|Z: C_{Z}(\bar{S})\right|=2$, then $\bar{Q} \cong Z_{3}$ and $[Z, \bar{Q}] \cong E_{4}$;

(ii) If $|\bar{S}|=4$, and $\left|Z: C_{Z}(\bar{S})\right| \leqslant 4$, then $\bar{Q} \cong Z_{3} \times Z_{3}$.

Proof. First we claim that if $\left|Z: C_{Z}(\bar{x})\right|=2$ for an involution $\bar{x} \in$ $\bar{S}^{\#}$, then $\bar{D}$, the normal closure of $\bar{x}$ in $|\bar{H}|^{*}$, is isomorphic to $S_{3}$ and further $Z=C_{Z}(\bar{D}) \times[Z, O(\bar{D})],[Z, O(\bar{D})] \cong E_{4}$. Clearly our claim will suffice for the proof of (i).

We proceed with the proof of our claim. Since $O_{2}\left(\bar{H}^{*}\right)=1$, we can find an $\bar{H}^{*}$-conjugate, $\bar{y}$, of $\bar{x}$ such that $[\bar{x}, \bar{y}] \neq 1$. Thus $\bar{F}=\langle\bar{x}, \bar{y}\rangle$ is a nonabelian dihedral group, and $O(\bar{F})$ acts faithfully on $Z / C_{Z}(\bar{F})$ which has order at most 4. $\bar{F}$ has abelian Sylow 2-subgroups and is itself nonabelian, so $O(\bar{F}) \neq$ 1 , and we must have $|O(\bar{F})|=3,\left|Z: C_{Z}(\bar{F})\right|=4$. It follows that $\bar{F} \cong S_{3}$ and $Z=C_{Z}(\bar{F}) \times[Z, O(\bar{F})]$ with $[Z, O(\bar{F})] \cong E_{4}$. It remains to show that $\bar{F}=\bar{D}$. Suppose that $\bar{t}$ is any conjugate of $\bar{x}$ in $\bar{H}^{*}$, and let $\bar{R}=\langle\bar{F}, \bar{t}\rangle$, $Y=C_{Z}(\bar{R})$. We know $|Z: Y| \leqslant 8$ whence $\widetilde{R}=\bar{R} / C_{\bar{R}}(Z / Y)$ is isomorphic to a subgroup of $G L(3,2)$. Also $C_{\bar{R}}(Z / Y)$ centralizes the series $1 \subseteq Y \subseteq Z$, so $C_{\bar{R}}(Z / Y) \subseteq O_{2}(\bar{R})$. Consequently $\widetilde{F} \cong S_{3}$. As $\widetilde{R}$ has abelian Sylow 2-subgroups, it follows from the subgroup structure of $G L(3,2)$ that $\widetilde{R}=\bar{F}$, whence $\bar{R}=\bar{F} O_{2}(\bar{R})$. 
Again because $\bar{H}^{*}$ has abelian Sylow 2-subgroups, $\bar{R}=\bar{F} \times O_{2}(\bar{R})$, and $O(\bar{F})=$ $O(\bar{D})$. Thus $\bar{t}$ inverts $O(\bar{F})$ and centralizes $C_{Z}(\bar{F})$, whence $\bar{D}$ centralizes $C_{Z}(\bar{F})$. We conclude $\bar{F}=\bar{D}$.

To prove (ii) we will show that $\left|Z: C_{Z}(x)\right|=2$ for some $\bar{x} \in \bar{S}^{\#}$. If not, then as $\bar{H}$ acts faithfully on $Z,\left|Z: C_{Z}(x)\right|=4$ for all $\bar{x} \in \bar{S}^{\#}$. Consequently $Y=C_{Z}(\bar{S})$ implies $Y=C_{Z}(\bar{x})$ for $\bar{x} \in \bar{S}^{\#}$. Thus $C_{\bar{Q}}(\bar{x})$ normalizes $Y$ whence $\bar{Q}=\left\langle C_{\bar{Q}}(\bar{x}) \mid \bar{x} \in \bar{S}^{\#}\right\rangle$ normalizes $Y$. As $\bar{S}$ centralizes $Z / Y$ and $Y$, so does $[\bar{Q}, \bar{S}] \stackrel{Q}{=} \bar{Q}$. Hence $\bar{Q}$ acts trivially on $Z$. By $(2.65), \bar{Q}=1$. But then $\bar{S}=\mathrm{O}_{2}(\bar{H})$ which is not the case. Thus $\left|Z: C_{Z}(\bar{x})\right|=2$ for some $\bar{x} \in \bar{S}^{\#}$.

Choose $\bar{x} \in \bar{S} \#$ so that $\left|Z: C_{Z}(\bar{x})\right|=2$, and let $\bar{D}$ be the normal closure of $\bar{x}$ in $\bar{H}^{*}$. Since $\bar{D} \cong S_{3}, \bar{H}^{*}=\bar{D} C_{\bar{H}^{*}}(\bar{D})$. Let $\bar{M}=C_{\bar{H}^{*}}(\bar{D}) . \bar{M}$ must centralize $[Z, O(\bar{D})]$ and act faithfully on $C_{Z}(\bar{D})=Z_{1}$. Since $|\bar{S}|=4$, $\bar{S} \cap \bar{M}$ contains an involution $\bar{t} .\left|Z: C_{Z}(\bar{S})\right| \leqslant 4$ implies $C_{Z_{1}}(\bar{t})$ has index 2 in $Z_{1}$, and we see from our initial claim that the normal closure of $\bar{t}$ in $\bar{M}$ is isomorphic to $S_{3}$. Thus the normal closure of $\bar{S}$ in $\bar{H}^{*}$ is isomorphic to $S_{3}$ $\times S_{3}$, and (ii) is valid.

(2.68) Assume the following conditions hold:

(a) $m(S) \leqslant 4$;

(b) $S$ is permutable with a cyclic 3-subgroup $R$ of $\mathrm{H}$ and $P=\mathrm{O}_{2}(S R)$ has index 2 in $S$;

(c) If $P_{0}=[P, R]$ and $P_{1}=C_{P}(R)$, then $P=P_{0} \times P_{1}$ and $P_{0}$ is a four subgroup of $Z$;

(d) $C_{S}(Z) \subseteq P$

(e) Some involution $z$ of $P_{1} \cap Z(S)$ is not isolated in $H$.

Under these conditions, we have

(i) $S-P$ contains an involution;

(ii) Either $m(Z(S))=3$ or $S \cong D_{8} \times D_{8}$ or $E_{4} \sim E_{2}$.

Proof. We have $Z=P_{0} \times Z_{1}$, where $Z_{1} \subseteq P_{1}$. Also $P_{0} \times\langle z\rangle \subseteq Z$ and so $Z \cong E_{8}$ or $E_{16}$ as $m(S) \leqslant 4$. Set $C=C_{H}(Z)$. We have that $T=S \cap$ $C \in S(C)$ and that $T \subseteq P$. Moreover, $T \subset S$, so $\bar{S} \neq 1$. Since $P_{0} \subseteq Z$, also $\bar{P}=\bar{P}_{1}$. Since $R$ centralizes $Z_{1}$, we also have that $\bar{R}$ is of order $3, C_{\bar{S}}(\bar{R})=$ $\bar{P}=\bar{P}_{1}$, and $|\bar{S}: \bar{P}|=2$.

Suppose first that $Z \cong E_{8}$, whence $\bar{H}$ is isomorphic to a subgroup of $G L(3,2)$. Since $\bar{R}$ centralizes $\bar{P}$ of index 2 in $\bar{S}$ and $\bar{S}$ is elementary abelian, the only possibility is that $\bar{P}=1$ and $\bar{H} \cong S_{3}$. But then $\bar{H}=\overline{R S}$ leaves $z$ invariant and so $z$ is isolated in $H$, contrary to assumption. Thus $Z \cong E_{16}$ and $\bar{H}$ is isomorphic to a subgroup of $G L(4,2)$.

If $\bar{H}$ were nonsolvable, the only possibilities for $\bar{H}$ would be $H \cong L_{2}(5)$ 
or $Z_{3} \times L_{2}(5)$. Since $\bar{R}$ is inverted by an element of $\bar{S}$, it follows in either case that $\bar{R} \subseteq L(\bar{H}) \cong L_{2}(5)$. However, by (2.66(i)), applied to the semidirect product of $Z$ and $\bar{L}(\bar{H}), Z$ is the natural module for $L(\bar{H})$ and as $\bar{R}$ normalizes some Sylow 2-subgroup of $L(\bar{H}), \bar{R}$ acts regularly on $Z^{\#}$ by (2.66(iii)) which is not the case. Thus $\bar{H}$ is solvable and as $\bar{S} \neq 1,|\bar{H}|$ is not divisible by 7 . If $|\bar{H}|$ were divisible by 5, the only possibility would be that $\bar{H}$ was dihedral of order 30 . But then $O(\bar{H})=$ $\bar{R} \times \bar{Q}$, where $\bar{Q} \cong Z_{5}$, and $\bar{Q}$ acts irreducibly on $Z$. However, $\bar{Q}$ leaves $P_{0}=$ $[Z, \bar{R}]$ invariant and $1 \subset P_{0} \subset Z$. Hence also $|\bar{H}|$ is not divisible by 5 and so $\bar{H}$ is a $\{2,3\}$ group.

We have that $\bar{H} \supset \overline{S R}$ and it follows now that $|\bar{H}|$ is divisible by 9 . Furthermore, either $O(\bar{H}) \cong Z_{3} \times Z_{3}$ is a Sylow 3-subgroup of $\bar{H}$ or $\bar{F}=$ $\mathrm{O}_{2}(\bar{H}) \cong E_{4}$. Consider the latter case. We have that $\bar{F} \subseteq \bar{P}=C_{\bar{S}}(\bar{R})$ and so $\bar{F}$ leaves both $Z_{1}$ and $P_{0}$ invariant and centralizes $P_{0}$. Since $\operatorname{Aut}\left(Z_{1}\right) \cong$ $G L(2,2)$, it follows that $\bar{F}_{1}=C_{\vec{F}}\left(Z_{1}\right) \neq 1$. But $\bar{F}_{1}$ centralizes $Z=P_{0} Z_{1}$, contrary to the fact that $\bar{H}$ acts faithfully on $Z$. Hence we have $O(\bar{H})=$ $Z_{3} \times Z_{3}$.

We write $O(\bar{H})=\bar{R} \times \bar{V}$, where $\bar{V}=Z_{3}$ and $\bar{V}$ centralizes $P_{0}$ and acts nontrivially on $Z_{1}$. Furthermore, $H=C N_{H}(T)$ by the Frattini argument and $T=P_{0} \times T_{1}$, where $T_{1}=T \cap P_{1}$. We conclude easily from these conditions that $N_{H}(T)$ contains a 3-subgroup $V$ which maps on $\bar{V}$, centralizes $P_{0}$, leaves $T_{1}$ invariant, and acts nontrivially on $Z_{1}$. These conditions imply that $Z_{1} \subseteq Z\left(T_{1}\right)$. Since $m(S) \leqslant 4$, it follows that $Z_{1}=\Omega_{1}\left(T_{1}\right)$ and as $V$ transitively permutes the involutions of $Z_{1}$, we also have that $T_{1}$ is a Suzuki 2-group. Since $S$ has class 2 , we easily check now that either (a) $T_{1} \cong E_{4}$ or (b) $|\bar{S}|=2$. Moreover, in either case, as $O(\bar{H})$ has no nontrivial fixed points on $T$, we obtain again by the Frattini argument that $S=T U$, where $U$ is elementary and $T \cap$ $U=1$. In particular, $S-P$ contains an involution.

Suppose first that $|\bar{S}|=2$. Then $\bar{S}=\bar{U}$ inverts $\bar{R}$ and so $U$ leaves both $P_{0}$ and $T_{1}$ invariant. Moreover, we can take $\bar{V}$ to be invariant under $\bar{U}$. If $\bar{U}$ inverted $\bar{V}$, we must have $T_{1}=Z_{1} \cong E_{4}$, otherwise it would follow immediately that $T_{1} U$ had class at least 3 , which is not the case. We conclude in this case that $S=Z U \cong E_{4} \sim E_{2}$. On the other hand, if $\bar{U}$ centralizes $\bar{V}$, then $\bar{U}$ must centralize $Z_{1}$ as $\bar{V}$ acts irreducibly and nontrivially on $Z_{1}$. Since $S=T U$, we see then that $Z_{1} \subseteq Z(S)$. Since also $P_{0} \triangleleft S$ and $U$ does not centralize $P_{0}$, we conclude that $m(Z(S))=3$. Hence one of the alternatives of (ii) holds when $|\bar{S}|=2$.

It thus remains to treat the case in which $|\bar{S}|>2$, whence $T_{1}=Z_{1}, T=$ $Z$ and $S=Z U$. Now $\bar{H}$ is isomorphic to a subgroup of a Sylow 3-normalizer in $A_{8} \cong G L(4,2)$, which has dihedral Sylow 2-subgroups of order 8. Since $\bar{U}=$ $\bar{S}$, it follows that $U \cong E_{4}$. Furthermore, as a Sylow 3-normalizer in $A_{8}$ is 
uniquely determined up to conjugacy, we conclude now that there are exactly two possible actions for $U$ on $Z$. Since $A_{10}$ contains a split extension of $E_{16}$ by a Sylow 3-normalizer in $A_{8}$, we see that $S=Z U$ is isomorphic to a subgroup of $A_{10}$. It is now easily verified that either $S \cong D_{8} \times D_{8}$ or $S$ is of type $A_{8}$. However, the latter case is excluded as $S$ has class 2 . Thus one of the alternatives of (ii) holds in this case as well.

Fusion. We require a number of general results about the fusion of 2-elements in a group $G$. In particular, we need Glauberman's $Z^{*}$-theorem [6] and Thompson's fusion lemma $[22,5.38]$, which we shall not bother to state explicitly. However, Goldschmidt's strongly closed abelian 2-subgroup theorem [11] and his refinement of Alperin's fusion theorem [8] will be more important for us. His first result yields:

(2.69) If a Sylow 2-subgroup $S$ of the simple group $G$ possesses a nontrivial abelian subgroup which is strongly closed in $S$ with respect to $G$, then $G$ is isomorphic to one of the following groups:

$$
L_{2}(q), q \equiv 3,5(\bmod 8), q>3, J_{1}, L_{2}\left(2^{n}\right), S z\left(2^{n}\right), U_{3}\left(2^{n}\right), n \geqslant 2 \text {, or a }
$$
group of Ree type of characteristic 3 .

We state his second result only in the case that $G$ has a Sylow 2-subgroup $S$ of class 2 with elementary center.

(2.70) Let $D$ be the set of normal subgroups $D$ of $S$ with the following properties:

(a) $N=N_{G}(D)$ is 2-constrained and $D$ is a Sylow 2-subgroup of $\mathrm{O}_{2^{\prime}, 2}(\mathrm{~N})$

(b) If $\bar{N}=N / D$, then either $|\bar{S}| \leqslant 2$ or $\bar{N} / O(\bar{N})$ contains a normal subgroup of odd index isomorphic to $L_{2}\left(2^{n}\right)$ for some $n \geqslant 2$. Under these conditions, $D$ is a conjugation family for $S$ in $G$.

In other words, the fusion in $G$ of subsets of $S$ is controlled by the set of normal subgroups $D$ of $S$ with the above properties. This particular case of Goldschmidt's result follows immediately from his general result together with (2.65) and the fact that any subgroup $D$ of $S$ with $C_{S}(D) \subseteq D$ is necessarily normal in $S$.

It is an immediate consequence of the definition that $D$ is invariant under conjugation by $N_{G}(S)$. We note also that these conditions imply that $C_{N}(D)=$ $O(N) \times Z(D)$ and that the results of the preceding subsection can be applied to the group $N / O(N)$.

We shall need a sharpening of these results in some low rank cases. We preserve the above notation and set $\bar{N}=N / D, \bar{N}^{*}=O^{2^{\prime}}(\bar{N})$, and $Z=$ $\Omega_{1}(Z(D))$.

(2.71) Assume that $|\bar{S}| \geqslant 4$. Then we have

(i) If $m(Z) \leqslant 6$, then $\bar{N}^{*} \cong L_{2}(4)$ or $L_{2}(8)$ and correspondingly 
$m(Z) \geqslant 4$ or $m(Z)=6$;

(ii) If $m(Z) \leqslant 5$, then $\bar{N}^{*} \cong L_{2}(4)$ and $m(Z) \geqslant 4$;

(iii) If $m(Z) \leqslant 4$, then $\bar{N}^{*} \cong L_{2}(4)$ and $Z(D)=Z \cong E_{16}$.

Proof. By (2.65(iv)), $\bar{N}^{*}$ acts faithfully on $Z$, so $\bar{N}^{*}$ is isomorphic to a subgroup of $G L(k, 2)$, where $k=m(Z) \leqslant 6$. Moreover, by (2.70) and our assumption on $|\bar{S}|$, we have that $\bar{N}^{*} / O\left(\bar{N}^{*}\right) \cong L_{2}\left(2^{n}\right)$ for some $n \geqslant 2$. But $O\left(\bar{N}^{*}\right)$ is isomorphic to a subgroup of $G L(6,2)$ and so $\left|O\left(\bar{N}^{*}\right)\right|$ divides $3^{3} \cdot 5 \cdot 7^{2} \cdot 31$ (as 3 divides $\left.\left|\bar{N}^{*} / O\left(\bar{N}^{*}\right)\right|\right)$. Since $L_{3}(3)$ is minimal simple, it follows at once that $C_{\bar{N}^{*}}\left(O\left(\bar{N}^{*}\right)\right)$ must cover $\bar{N}^{*} / O\left(\bar{N}^{*}\right)$. But the groups $L_{2}\left(2^{n}\right)$ have no nontrivial central extensions by a group of odd order as their Sylow 2-subgroups for all odd primes are cyclic. Hence $\bar{N}^{*}=\bar{L} \times O\left(\bar{N}^{*}\right)$, where $\bar{L} \cong L_{2}\left(2^{n}\right)$. But $O^{2^{\prime}}\left(\bar{N}^{*}\right)=\bar{N}^{*}$ as $\bar{N}^{*}=O^{2^{\prime}}(\bar{N})$ and this forces $\bar{N}^{*}=\bar{L}$.

From the minimum degrees of the representations of $L_{2}\left(2^{n}\right)$, it follows now that $n=2$ or 3 and that $k \geqslant 2 n$. Furthermore, if $k=4, \bar{N}^{*}$ acts irreducibly on $Z$, which forces $Z(D)$ to be homocyclic. But $\bar{N}^{*}$ acts trivially on $\mho^{1}(D)$ by (2.65(iii)) and as $\bar{N}^{*}$ acts nontrivially on $Z$, we conclude that $Z(D)$ $=Z \cong E_{16}$. All parts of (2.71) now follow.

Some classification theorems. Finally we shall need certain general classification theorems concerning simple groups with specified Sylow 2-subgroups. We restrict ourselves to the case in which our simple group $G$ has Sylow 2-subgroup $S$ of class 2 .

First the main result of [1] yields

(2.72) If $m(S) \leqslant 2$, then $G \cong L_{2}(q), q \equiv 7,9(\bmod 16), A_{7}$, or $U_{3}(4)$.

Applying [17], we obtain that $G$ does not have product fusion.

(2.73) $S$ cannot be written as the direct product of two nontrivial subgroups, each strongly closed in $S$ with respect to $G$.

(2.74) $S$ is not isomorphic to $D_{8} \times E_{2}, D_{8} \times D_{8}, D_{8} \times Z_{2^{n}} \times Z_{2^{n}}, n \geqslant$ 1, $E_{2} m \sim E_{2}, m \geqslant 2$, and $S$ does not possess a subgroup of index 2 isomorphic to $E_{4} \times Z_{4}$.

Proof. Since $G$ is simple, the first and last cases are excluded by a result of Fong [5], while the main result of [16] excludes the second and third cases. Finally [11, Section 9, Corollary 6] rules out $S=E_{2^{m}} \sim E_{2}$.

3. The minimal counterexample. Henceforth $G$ will denote a minimal counterexample to the Main Theorem.

We first prove

Proposition 3.1. If $S$ is a Sylow 2-subgroup of $G$, then we have:

(i) Every proper subgroup of $G$ is a $C_{2}$-group; 
(ii) $Z(S)$ is elementary;

(iii) $m(S) \geqslant 3$.

(iv) $S$ contains no nontrivial abelian subgroup which is strongly closed in $S$ with respect to $G$.

Proof. If $H$ is a proper subgroup of $G$, then a nonsolvable composition factor $K$ of $H$ has Sylow 2-subgroups of class 1 or 2 . In the first case, the known structure of groups with abelian Sylow 2-subgroups implies that $K \in A$; while in the second case, the minimality of $G$ implies that $K$ satisfies the conclusion of the Main Theorem and again $K \in A$. Since $H$ also has Sylow 2-subgroups of class 1 or $2, H$ is therefore a $C_{2}$-group. Thus (i) holds. Furthermore, (ii) follows from (2.1). If $m(S) \leqslant 2$, then by (2.72) $G$ satisfies the conclusion of the Main Theorem and so is not a counterexample. Hence (iii) also holds. Finally if (iv) is false, $G \cong S z\left(2^{n}\right)$ or $U_{3}\left(2^{n}\right)$ by (2.69) and again $G$ is not a counterexample. Thus (iv) also holds.

We state two further properties of $G$.

Proposition 3.2. The following conditions hold:

(i) If $L_{1}(G)$ is empty or consists of an evenly embedded $L_{2}(5)$, then $O\left(C_{G}(x)\right)=1$ for every $x$ in $I(G)$.

(ii) If $L(G)$ is empty, then $H$ is 2-constrained with $O(H)=1$ for every 2-local subgroup $H$ of $G$.

Proof. By Proposition 3.1, we have $m(G) \geqslant 3$. Hence this proposition follows at once from (2.64).

4. Special linear components. The bulk of this paper consists of demonstrating that $L(G)$ is empty; in fact, all but the final section is devoted to the proof of this assertion.

We shall therefore now make the assumption

$$
L(G) \text { is nonempty. }
$$

Ultimately we shall show that this leads to a contradiction.

In this section, we prove

Proposition 4.1. No element of $L(G)$ is of type $S L(2, q), q$ odd.

Assume false and let $x \in I(G)$ be such that $\Lambda_{x}$ has a component $K$ of type $S L(2, q), q$ odd. We first prove

LEMMA 4.2. If $R \in S\left(C_{G}(x)\right)$, then we have

(i) $R$ normalizes $K$, and $\mho^{1}(R)$ centralizes $K / O(K)$;

(ii) If $y \in I(R)$ and $y$ centralizes $K / O(K)$, then $L_{2^{\prime}}\left(C_{K}(y)\right)$ lies in a component of $\Lambda_{y}$ of type $S L(2, q)$. 
Proof. Since $K$ has nonabelian Sylow 2-subgroups, $R$ normalizes $K$ by (2.13). Setting $H=C_{G}(x)$ and $\bar{H}=H / O(H)$, it follows from (2.18) that $\left|N_{\bar{H}}(\bar{K}) / \bar{K} C_{\bar{H}}(\bar{K})\right|$ is odd, whence $\bar{R} \subseteq \bar{K} C_{\bar{H}}(\bar{K})$. But $\bar{R} \cap \bar{K}$ is a quaternion group and so $\mho^{1}(\bar{R}) \subseteq C_{\bar{H}}(\bar{K})$. Thus both parts of (i) hold.

Next let $y$ be as in (ii). Setting $K_{0}=L\left(C_{K}(y)\right),(2.42)$ implies that $K_{0} \subseteq$ $L_{y}$ and so by (2.43) the normal closure $L$ of $K_{0}$ in $L_{y}$ either consists of a single component or two components interchanged by $x$. Clearly these components have nonabelian Sylow 2-subgroups and so the second possibility is excluded by (2.13). Thus $L$ is a component of $L\left(C_{\bar{L}}(x)\right)$ and consequently $\bar{K}_{0}=\bar{L}$ by $(2.27(\mathrm{v}))$, Hence $L \cong K / O(K)$ and so $L$ is of type $S L(2, q)$, proving (ii).

Now we can establish the proposition. Choose $z$ in $I(K)$, so that $z$ centralizes $K / O(K)$ and $z \in L_{2^{\prime}}\left(C_{K}(z)\right)$. Hence by the preceding lemma, $z \in L$ for some component $L$ of $\Lambda_{z}$ of type $S L(2, q)$. Let $J$ be the product of all components of $\Lambda_{z}$ of type $S L(2, q)$, set $H=C_{G}(z)$, and let $S \in S(H)$. Since $z$ lies in a quaternion subgroup of $L, z \in S^{\prime}$ and so $S \in S(G)$. Finally let $E$ be the subgroup of $S$ which projects onto $Z(J / O(J))$. Then $E$ is elementary and $z \in E$. Moreover, $E \subseteq S^{\prime}$ and so $E \subseteq Z(S)$.

We shall argue that $E$ is strongly closed in $S$ and this will contradict Proposition 3.1. Let $t \in E$ and suppose $y=t^{g}, g \in G$, is a conjugate of $t$ which lies in $S$. We must prove that $y \in E$. Setting $M=L\left(C_{J}(t)\right)$, we see that $M$ covers $J / O(J)$ and so we can apply Lemma 4.2(ii) to each component of $H$ which lies in $J$ to conclude that $M$ lies in a product $N$ of components of $\Lambda_{t}$, each of type $S L(2, q)$. Another application of the same lemma to the components of $N$ and the involution $z$ yields that $M$ also covers $N / O(N)$. In particular, $N^{g}$ is a product of components of $\Lambda_{y}$ of type $S L(2, q)$ and $E^{g}$ projects onto $Z\left(N^{g} / O\left(N^{g}\right)\right)$.

On the other hand, $y$ leaves each component $J_{i}$ of $J$ invariant by (2.13) and so $y$ normalizes $R_{i}=S \cap J_{i} \cong Q_{8}, 1 \leqslant i \leqslant n$. Since $R_{i}\langle y\rangle$ is of class 2 , $y$ centralizes an element $u_{i}$ of $R_{i}$ of order 4 and $\left\langle u_{i}^{2}\right\rangle=Z\left(R_{i}\right) \subseteq E, 1 \leqslant i \leqslant$ $n$. Clearly we have, in fact, $E=\left\langle u_{i}^{2} \mid 1 \leqslant i \leqslant n\right\rangle$. It follows therefore that $E \subseteq$ $\mho^{1}\left(C_{S}(y)\right)$. Hence if $Q$ is a Sylow 2-subgroup of $C_{G}(y)$ containing $C_{S}(y)$, then $E \subseteq \mho^{1}(Q)$ and so $E$ centralizes $N^{g} / O\left(N^{g}\right)$ by Lemma 4.2(i). In particular, $z$ centralizes this section and so if we set $V=L\left(C_{N^{g}}(z)\right)$, we have that $V$ covers $N^{g} / O\left(N^{g}\right)$. Applying Lemma 4.2(ii) once again and using the definition of $J$, we obtain now that $V \subseteq J$. But $V$ and $J$ consists of the same number of components as $J$ and $N$ do and consequently $V$ covers $J / O(J)$.

Finally, $y \in E^{g}$ projects into $Z\left(N^{g} / O\left(N^{g}\right)\right)$ and as $y$ centralizes $z$, it follows that $y \in V$ and $y$ projects into $Z(V / O(V))$. Since $V$ covers $J / O(J)$, 
this implies that $y$ projects into $Z(J / O(J))$. However, $y \in S$ and $E$ is precisely the subgroup of $S$ which projects onto $Z(J / O(J))$. We conclude that $y \in E$, as required, and Proposition 4.1 is proved.

5. Low rank cases. In this very long section, we shall establish a large number of results in the case $m(G) \leqslant 6$. In particular, we shall show (under our assumption $L(G)$ nonempty) that $m(G)$ is necessarily at least 5 . The analysis will be almost entirely fusion-theoretic. Whenever $S$ is a Sylow 2-subgroup of $G, D$ will always denote the Goldschmidt conjugation family (2.70) for $S$ in $G$.

We begin with two preliminary results.

Lemma 5.1. If $m(G) \leqslant 6$ and $L_{x}$ is of type $L_{2}(16)$ for $x$ in $I(G)$, then no involution of $C_{G}(x)$ acts irregularly on $L_{x}$. In particular, if $m(G) \leqslant$ 6 , then $G$ is $\Lambda$-balanced.

Proof. Assume false and choose $x$ to violate the conclusion so that $P \in$ $S\left(C_{G}(x)\right)$ has maximal order. We set $K=L_{x}, T=P \cap K, R=C_{P}(K / O(K))$ and we let $S \in S(G)$ with $S \supseteq P$. Also let $y \in I(P)$ act irregularly on $K$. Note that by Lemma (2.19) we have $|P: T R|=2$.

Consider first the case $P \subset S$. We claim first that $P \in S\left(C_{G}(a)\right)$ for all $a$ in $I(Z(P) \cap R)$. Indeed, as $J=L_{2},\left(C_{K}(a)\right)$ covers $K / O(K),(2.43)$ implies that the normal closure $L$ of $J$ in $L_{a}$ consists of either one component or two components interchanged by $x$ and that $J$ projects onto $L\left(C_{L / O(L)}(x)\right)$. Hence by (2.26), $L$ is either of type $L_{2}(16), L_{2}\left(2^{8}\right)$, or consists of two components of type $L_{2}(16)$. However, the last two cases are excluded by our assumption $m(G) \leqslant 6$. Thus $L$ is of type $L_{2}(16)$ and so $J$ covers $L / O(L)$. But $P \subseteq C_{G}(a)$ as $a \in Z(P)$. In particular, $y \in C_{G}(a)$ and we see that $y$ acts irregularly on $L$. Now our assertion follows from our maximal choice of $P$. In particular, as $P \subset S$, we have $R \cap Z(S)=1$.

Since $\mho^{1}(R) \subseteq Z(S)$ by $(2.1)$, it follows now that $R$ is elementary. Likewise as $R \triangleleft P$ and $[R, P] \subseteq Z(S)$, we also have that $R \subseteq Z(P)$. Furthermore, since $T \cong E_{16}$ and $T \cap R=1$, we have $R \cong E_{2}$ or $E_{4}$ as $m(G) \leqslant 6$. Setting $E=C_{T}(y)$, we have that $E=[T, y] \cong E_{4}$ and $E \subseteq Z(S)$. On the other hand, $Z(S) \subseteq Z(P)=E \times R$ and so $Z(S)=E$. Moreover, $P \triangleleft S$ as $Z(S) \subseteq P$. Since $Z(P)=E \times R$ and $C_{S}(a)=P$ for $a$ in $R^{\#}$, we also obtain that $|S: P|=2$ or 4 .

We shall now contradict this fact. First of all, suppose $R \cap R^{w} \neq 1$ for some $w$ in $S-P$. Then there exist $u, v$ in $R^{\#}$ with $u=v^{w}$ and we have $[v, w] \subseteq R \cap S^{\prime} \subseteq R \cap Z(S)=1$. Thus $w$ centralizes $v$ and so $\langle P, w\rangle \subseteq$ $C_{G}(v)$, contrary to the fact that $P \in S\left(C_{G}(v)\right)$. Hence $R \cap R^{w}=1$ for $w$ in $S-P$.

Now set $N=N_{G}(T R)$ and consider the conjugacy classes of $(T R)^{\#}$ in $N$. 
By the structure of $K, N$ contains a cyclic subgroup $F$ which acts transitively on $T^{\#}$ and centralizes $R$. Hence $F$ partitions $T R-R$ into disjoint orbits of size $15=|T|-1$. Furthermore, $T R \triangleleft S$ as $Z(S) \subseteq T R$, so $S \subseteq N$. Take $w$ in $S-P$. Since all involutions of $T$ are conjugate, they are all central involutions. However, the involutions of $R$ are noncentral. Hence $R \cap T^{w}=1$. Since $R \cap R^{w}=1$, it follows that each element of $R^{\#}$ is fused to a single $F$-orbit of $T R-T$. This in turn implies that the conjugacy class of $x$ in $N$ consists of $16 k$ elements for some $k$. Since $P \in S\left(C_{G}(x)\right)$ and $S \in S(N)$, we conclude that $|S: P|$ is divisible by 16 . This contradiction eliminates the possibility $P \subset S$ and so we have $S=P$.

By Thompson's fusion lemma, $y$ has a conjugate $u$ in TR. We can assume that $y \sim u$ in $N=N_{G}(D)$ for some $D$ in the Goldschmidt conjugation family $D$ with $\langle y, u\rangle \subseteq D ; N=N^{*} N_{N}(S)$, where $N^{*}=O^{2}(N)$. We argue that we can assume the conjugacy of $y$ and $u$ occurs in $N^{*}$. Since $u \in \Omega_{1}(T R)$ and $y \notin T R$, we need only show that $\Omega_{1}(T R)$ is characteristic in $S$. But $m(R)=1$ or 2 as $m(G) \leqslant 6$. Hence if $v \in I(T R)$, we see that $m\left(C_{S}(v)\right) \geqslant 4+m(R)$ as $v$ centralizes $T$. On the other hand, if $v \in(S-T R)$, then $m\left(C_{S}(v)\right) \leqslant 1+$ $m\left(C_{T}(v)\right)+m(R)=3+m(R)$ and the desired assertion follows.

By (2.65), $N^{*}$ centralizes $D / Z$, where $Z=\Omega_{1}(Z(D))$, and consequently $y=u z$ for some $z$ in $Z$. This yields that $z \notin T R$. Since $D \subseteq C_{S}(z)$, this implies that $D \cap T \subseteq E R$, where, as above, $E=C_{T}(y) \subseteq Z(S)$. In particular, $|S: D| \geqslant 4$. On the other hand, $m(D) \leqslant m\left(C_{S}(z)\right) \leqslant m(E)+1+m(R) \leqslant 5$. Now (2.71) forces $|S: D|=4$ and we conclude that $S=D T$ with $T \cap D=E$. Again by (2.71), $\bar{N}^{*}=N^{*} / D \cong L_{2}(4)$. Our conditions imply that $\bar{T} \in$ $S\left(\bar{N}^{*}\right)$. But $\bar{T}$ centralizes $Z_{0}=Z \cap T R$ as $T R=T \times R$ and so $\left|Z: Z_{0}\right| \geqslant$ 4 by (2.66). But $\left|Z: Z_{0}\right| \leqslant 2$ as $|S: T R|=2$. This contradiction completes the proof.

We first treat the case of noncentral involutions. We need two results, which we shall prove simultaneously.

Proposition 5.2. The following conditions hold in $G$ :

(i) If $L_{\boldsymbol{x}}=1$ for all $x$ in $I(G)$ such that $m\left(C_{G}(x)\right) \geqslant 5$, then $m(G)$ $\leqslant 4$ and $L_{z} \neq 1$ for some central involution $z$ of $G$;

(ii) If $m(G)=5$ or 6 and $L_{2}(9) \in L_{2}(G)$, then for some $z$ in $I(G)$ such that $m\left(C_{G}(z)\right)=m(G), L_{z}$ has a component of type $L_{2}(9)$.

We assume false and proceed in a sequence of lemmas to derive a contradiction. By our basic assumption, $L(G)$ is nonempty and so $L_{x} \neq 1$ for some $x$ in $I(G)$ in case (i). Likewise in case (ii), $L_{x}$ has a component of type $L_{2}(9)$ for some $x$ in $I(G)$. In each case we choose $x$ so that a Sylow 2-subgroup $P$ of $C_{G}(x)$ has maximal order and we let $S$ be a Sylow 2-subgroup of $G$ con- 
taining $P$. Since the proposition is assumed false, we have $P \subset S$ in all cases. Moreover, $m(P) \leqslant 4$ in case (i) and $m(P)<m(G)=5$ or 6 in case (ii). According as we are in case (i), or (ii), we set $K=L_{x}$, or $K=$ the product of all components of $L_{x}$ of type $L_{2}(9)$. Then $K \neq 1$ in both cases. In addition, we set $T=P \cap K$ and $R=C_{P}(K / O(K))$. We fix all this notation.

We first prove

Lemma 5.3. $K$ consists of a single component of type $L_{2}(q), q$ odd, $A_{7}$, $U_{3}(4), J_{1}, L_{2}(8), S z(8), U_{3}(8)$, or of Ree type.

Proof. Let $J$ be a component of $K$ and set $\bar{J}=J / O(J)$. By Proposition 4.1 , we have $\bar{J} \nRightarrow S L(2, q), q$ odd. Furthermore, in case (i), $m(K) \leqslant 4$ and so $\bar{J}$ is not a nontrivial central extension of $L_{3}(4)$ by (2.16). Hence $\bar{J}$ is simple in both cases and so $x \notin J$. Likewise $m(J) \leqslant 3$ in case (i). It follows at once now that $J$ is of one of the types listed. Hence if $J=K$, the lemma holds.

Suppose then that $K$ has a second component $L$. Then similar conclusions hold for $L$, so that $L / O(L)$ is simple and $x \notin L$. This implies that $m\left(C_{G}(x)\right)$ $\geqslant m(J)+m(L)+1 \geqslant 5$. Hence equality holds throughout and $m(J)=m(L)=$ 2. In particular, we are necessarily in case (ii).

Suppose $S \cap L$ contained a central involution $y$. Since $y$ centralizes $J / O(J)$, it follows from $L$-balance and (2.26) that $L_{y}$ has a component of type $L_{2}(9)$. Since $C_{G}(y)$ contains a Sylow 2-subgroup of $G$, our maximal choice of $x$ and $P$ is contradicted. Hence no involution of $S \cap L$ is central; and, in particular, $S \cap L$ must be abelian, contradicting $L \cong L_{2}(9)$.

We next prove

LEMMA 5.4. The following conditions hold:

(i) $R \cap Z(S)=1$;

(ii) $R \subseteq \Omega_{1}(Z(P))$;

(iii) $R \cap R^{w}=1$ for $w$ in $S-P$;

(iv) If $T \cap R^{w} \neq 1$ for some $w$ in $S-P$, then $T$ is elementary and $T \cap Z(S)=1$;

(v) $P \triangleleft S$.

Proof. If $y \in I(R)$, then $L_{2}\left(C_{K}(y)\right)$ covers $K / O(K)$. In case (i), $L$-balance implies that $L_{y} \neq 1$, and in case (ii), (2.43) and (2.26) imply that $L_{y}$ has a component of type $L_{2}(9)$. Hence if $y \in Z(P)$, our maximal choice of $P$ forces $P \in S\left(C_{G}(y)\right)$. In particular, $y \notin Z(S)$ as $P \subset S$, so (i) holds. Moreover, (ii) and (iii) now follow exactly as in the proof of Lemma 5.1. Likewise $P \triangleleft S$ as $Z(S) \subseteq P$, so (v) also holds.

Finally as $\mho^{1}(T) \subseteq Z(S)$, either $T$ is elementary with $T \cap Z(S)=1$ or 
$T$ contains an involution $t$ of $Z(S)$. But all involutions of $K$ are conjugate by the structure of $K$. Hence if $T \cap R^{w} \neq 1$ for some $w$ in $S-P$, then $t \sim y$ for some $y$ in $R$ and so $y$ would be a central involution of $G$. But as we have seen, $L_{y} \neq 1$, or $L_{y}$ has a component of type $L_{2}(9)$, as the case may be. Hence our maximal choice of $\boldsymbol{P}$ and $\boldsymbol{x}$ would be contradicted. Thus (iv) also holds.

We shall divide the analysis into two subcases:

Subcase (a). $C_{P}(T) \subseteq T R$;

Subcase (b). $C_{P}(T) \nsubseteq T R$.

Lemmas 5.5-5.8 are proved under the assumption $C_{P}(T) \subseteq T R$.

Lemma 5.5. We have $T \cap R^{w}=1$ for $w$ in $S-P$.

Proof. Suppose false, in which case $T$ is elementary and $T \cap Z(S)=1$ by the preceding lemma. Consequently $T \cap P^{\prime}=1$ and now (2.18) yields $P=$ $T R=T \times R$. Since $P \triangleleft S$ and $R \cap Z(S)=1$, also $R$ is elementary. Furthermore, as all involutions of $T$ are conjugate in $K$ and $T \cap R^{w} \neq 1$, they all are conjugate to involutions of $R$. In particular, no involution of $T$ is central.

We claim next that $T \cap T^{w}=1$ for any $w$ in $S-P$. Indeed, if false, then the argument of Lemma 5.1 (with the roles of $T$ and $R$ interchanged) yields that $w$ centralizes some involution $t$ of $T$. But then $\left|C_{S}(t)\right|>|R T|=$ $|P|$. However, as $t$ is conjugate to an involution of $R$, our maximal choice of $P$ is contradicted. Thus $T \cap T^{w}=1$, as asserted.

We have that $w$ normalizes $P$ and $m(P) \leqslant 5$. Since $R \cap R^{w}=T \cap$ $T^{w}=1$, the only possibility is that $R \cong T \cong E_{4}$, and $[R, w] \cong E_{4}$. In particular, $m(Z(S)) \geqslant 2$. Since $Z(S) \subseteq P$ with $Z(S) \cap R=Z(S) \cap T=1$, we have, in fact, $Z(S) \cong E_{4}$.

Now $K$ contains a 3-element $v$ which acts nontrivially on $T$ and trivially on $R$.

Considering the action of $v$ on $Z(S)$, we see that $P$ contains at least 9 central involutions of $G$. Since none of the involutions of $R$ or $T$ is central, it follows that $I(R \cup T)$ consists precisely of the noncentral involutions of $G$ which lie in $P$. Since $R \cap R^{w}=T \cap T^{w}=1$ for $w$ in $S-P$, we conclude that any such $w$ must interchange $R$ and $T$, whence $|S: P|=2$. Thus, in fact $S=P\langle w\rangle$ and we can choose $w$ to be an involution. Therefore $S \cong$ $E_{4} \sim E_{2}$, contrary to (2.74).

LEMMA 5.6. The following conditions hold:

(i) $\Omega_{1}(T)=Z(S)=[S, x]$;

(ii) $P=R T$; 
(iii) $|R| \leqslant|Z(S)|$;

(iv) $|S: P|=|Z(S)|$;

(v) $m(S) \leqslant 2 m(Z(S))$.

Proof. We know already that $R$ is elementary. Hence if $B_{1} \in M(T)$ and we set $B=B_{1} R$, we have that $B \in M(T R)$. We set $Z=Z(S)$ and $N=$ $N_{G}(B)$. Since $Z \subseteq P$ and $C_{P}(T) \subseteq R T$ by assumption, we have $Z \subseteq R T$, whence $Z \subseteq B$. Thus $B \triangleleft S$ and so $S \subseteq N$. Furthermore, by the structure of $K, N$ contains an element $v$ which acts transitively on $B_{1}^{\#}$ and trivially on $R$.

We consider the conjugacy classes of $B^{\#}$ under the action of $N$. By the action of $v$ on $B$, the elements of $B_{1}^{\#} y$ are all conjugate in $N$ for a fixed $y$ in $R^{\#}$. Taking $w$ in $S-P$, we know that $R \cap R^{w}=1$ and $B_{1} \cap R^{w}=1$ by Lemmas 5.4(iii) and 5.5. This implies that $R^{w} \cap B_{1}^{\#} y$ consists of a single element for each $y$ in $R$. If this element is $b^{w}$ with $b$ in $R^{\#}$ then the elements $\{b\} \cup B_{1}^{\#} y$ are all conjugate in $N$ and this set has order $2^{n}=\left|B_{1}\right|$. Furthermore, the involutions of $B-B_{1}$ are all noncentral in $G$, so $Z \subseteq B_{1}$. Since $v$ acts transitively on $B_{1}^{\#}$, all involutions of $B_{1}$ are central in $G$, so no element of $B-B_{1}$ is conjugate to an element of $B$. We conclude that the $N$-classes of $B^{\#}$ consist of $B_{1}^{\#}$ and unions of subsets of $B-B_{1}$, each of size $2^{n}$.

Clearly $x \in R$ and our argument yields that $\left|N: C_{N}(x)\right|$ is divisible by $2^{n}$. Thus $|S: P|$ is divisible by $2^{n}$ and consequently so is $|[S, x]|$. But $[S, x] \subseteq B \cap Z$ and so $|Z| \geqslant 2^{n}$. However, $Z \subseteq B_{1}$ and $\left|B_{1}\right|=2^{n}$. We therefore conclude that $B_{1}=Z=[S:\langle x\rangle]$. In particular, $B_{1} \subseteq Z(T)$ and so $T$ is not dihedral of order 8. But now considering the possibilities for $K$ as listed in Lemma 5.3, we conclude at once that $\Omega_{1}(T)=B_{1}=Z$.

Thus $B=Z \times R$. Since $R \cap R^{w}=1$ for $w$ in $S-P$ and $B \triangleleft S$, it follows now that $|R| \leqslant|Z|$. Furthermore, if $m(T)=3$, then by Lemma 5.3 and (2.18), we have $P=T R$. The same conclusion holds if $K$ is of type $U_{3}$ (4) by (2.18). The only other possibility is that $T=Z \cong E_{4}$. Since $C_{P}(T) \subseteq T R$, we reach the same conclusion in this case as well. Thus all parts of (i), (ii), and (iii) hold. Furthermore, as $[S, x] \subseteq Z$, which has order $2^{n}$, and $C_{S}(x)=P$, we have $|S: P| \leqslant 2^{n}$. Since we have already established the reverse inequality, (iv) also holds.

Finally let $A \in M(S)$. If $A \subseteq P$, then clearly $A=B=Z R$ and so by (iii), $m(A) \leqslant 2 m(Z)$. Thus (v) holds in this case. Suppose $A \nsubseteq \subseteq P$. We have $Z \subseteq A$ and $A \cap P \subseteq B$. Since $R \cap R^{w}=1$ for $w$ in $S-P$, it follows that $A \cap P=Z$. But $|A: A \cap P| \leqslant|Z|$ by (iv) and so (v) holds in this case as well. 
Proof. Suppose false. Since $\Omega_{1}(T)=Z=Z(S)$ is elementary by the preceding lemma, $K$ is necessarily of type $U_{3}(4), U_{3}(8)$ or $S z(8)$. In particular, we are in case (i). Moreover, if $|Z|=2^{n}$, we have $n=2$ or 3 , and again by the preceding lemma, we have correspondingly $m(S) \leqslant 4$ or 6 .

By Proposition 3.1, $Z$ is not strongly closed in $S$ and so $z \sim y$ in $G$ for some $z \in Z$ and $y \in S-Z$. The argument of the last lemma showed that all involutions of $Z R-Z$ are noncentral. Since $\Omega_{1}(P)=Z R$ in the present case, it follows that $y \in S-P$.

By (2.70), we can assume that $z \sim y$ in $N=N_{G}(D)$ for some $D$ in $D$. We have that $N=N^{*} N_{N}(S)$, where $N^{*}=O^{2}(N)$. Since $N_{N}(S)$ leaves $Z=$ $Z(S)$ invariant, we can suppose without loss that $z \sim y$ in $N^{*}$. Since $z \in$ $Z(D)$, it follows that also $y \in Z(D)$. Thus $D \subseteq C_{S}(y)$. But if $a \in Z R-Z$, $a=z_{1} r$ with $z_{1} \in Z$ and $r \in R^{\#}$. Since $Z=Z(S)$, it follows that $C_{S}(\bar{a})=$ $C_{S}(r)=P$. Since $y \in S-P$, we conclude that $D \cap Z R=Z$. In particular, $R \cap D=1$.

We use this to prove that $|S: D|>2$. Suppose false. Since $R \cap D=1$, this forces $R=\langle x\rangle \cong E_{2}$ and likewise we have $|T R: D \cap T R| \leqslant 2$. Together these conditions imply that $D$ covers $T R / R$ and so by the structure of $T$, it follows that $Z \subseteq D^{\prime}$. But $N^{*}$ acts trivially on $D^{\prime}$ by (2.65) and so $z$ cannot be conjugate to $y$ in $N^{*}$. Thus $|S: D|>2$, as asserted.

Now set $E=\mho^{1}(D)$. We have that $m(S) \leqslant 4$ or 6 according as $|Z|=$ 4 or 8 . We can apply (2.71) and (2.66) to conclude that $|S: D| \leqslant|Z: E| \leqslant$ $|Z|$.

On the other hand, $|P: Z| \geqslant|Z||R|$, by the structure of $T$, so $|P: Z|>$ $|Z|$. Since $|S: D| \leqslant|Z|$, it follows that $P \cap D \supset Z$. Since $\Omega_{1}(P)=Z R$ and $Z R \cap D=Z$, we see that $P \cap D$ contains an element of order 4. Thus $E \neq 1$ and as $|Z: E| \geqslant|S: D| \geqslant 4$, we must have equality throughout with $|Z|=8$ and $|E|=2$. However, as $|S: D|=4,|P: P \cap D| \leqslant 4$. According as $K$ is of type $S z(8)$ or $U_{3}(8),|P| \geqslant 2|Z|^{2}$ or $2|Z|^{3}$ and consequently $|P \cap D| \geqslant$ $4|Z|$ or $4|Z|^{2}$ respectively. But $P \cap D$ is isomorphic to a subgroup of $T$ containing $Z$ inasmuch as $Z R \cap D=Z$ and $P=T \times R$. It follows directly now from the structure of a 2-group of type $S z(8)$ or $U_{3}(8)$ that $\left|\mho^{1}(P \cap D)\right| \geqslant 4$ whence $|E| \geqslant 4$. This contradiction establishes the lemma.

Finally we can eliminate the case $C_{P}(T) \subseteq T R$.

Lemma 5.8. We have $C_{P}(T) \nsubseteq T R$.

Proof. Note first that as in the preceding lemma, $z \sim y$ in $G$ for some $z$ in $T^{\#}$ and $y$ in $S-P$ and that this conjugation can be taken to occur in $N^{*}=O^{2^{\prime}}\left(N_{G}(D)\right)$ for some $D$ in $D$. 
By Lemmas 5.6(i) and 5.7, $T=Z(S)$. We know that $K$ contains a cyclic subgroup $F_{1}$ which acts transitively on $T^{\#}$ and centralizes $R$. Then $F_{1}$ normalizes $H=C_{G}(T) \cap N_{G}(P)$. As $S C_{G}(P)=O_{2}\left(H / C_{G}(P)\right)$, it follows by the Frattini argument that $N_{G}(S)$ contains a cyclic subgroup $F$ which acts transitively on $T^{\#}$ and centralizes $R$. Setting $F_{0}=C_{F}(T)$, we have that $F / F_{0}$ is cyclic of order $|T|-1=2^{n}-1, n=2$ or 3 , and that $F_{0}$ centralizes $P=$ $T R=C_{S}(x)$. Thompson's $(A \times B)$-lemma now yields that $F_{0}$ centralizes $S$. Since $|S / P|=2^{n}$ and $[S,\langle x\rangle]=T$, it also follows that $F / F_{0}$ acts transitively on $(S / P)^{\#}$. Since $S / T$ is elementary, this in turn implies that $S / T=Q / T \times$ $P / T$, where $Q / T$ is elementary or of order $2^{n}$ and $F$ acts transitively on $(Q / T)^{\#}$.

If $Q-T$ contains an involution, then every coset of $T$ in $Q$ contains an involution and it follows that $Q$ is elementary of rank $2^{n}$. In the contrary case, $\Omega_{1}(Q)=T$ and so our involution $y$ has the form $y=y_{1} y_{2}$ with $y_{1} \in$ $Q-T$ and $y_{2} \in R^{\#}$. Since $y^{2}=1, y_{2}$ must then invert $y_{1}$. Conjugating by the elements of $F$, it follows that $y_{2}$ must invert $Q$. Thus $Q$ is again abelian and in this case $Q$ is homocyclic of exponent 4 and rank $n$.

We next eliminate the latter case. Since $C_{Q}(a)=T$ for $a$ in $R^{\#}, y_{2}$ is the only involution of $R$ which inverts $Q$. This implies that all involutions of $S-P$ lie in the set $(Q-T) y_{2}$. But $y \in Z=\Omega_{1}(Z(D))$ as $z \in T \subseteq Z$ and $y \sim z$ in $N^{*}$. Furthermore, $D \cap Q=T$ as $y$ inverts $Q$ and centralizes $D$. Our argument thus yields that $Z=T\langle y\rangle$ is of rank $n+1$ and that $|S / D| \geqslant$ $2^{n}$. However, as $n=2$ or 3 , this contradicts (2.71).

There remains the case that $Q$ is elementary. This time we use the fact that $Q$ is not strongly closed in $S$, whence $u \sim v$ in $G$ for some $u$ in $Q$ and $v$ in $S-Q$. Thus $v=v_{1} v_{2}$ with $v_{1} \in Q$ and $v_{2} \in R^{\#}$. Again $v_{2}$ inverts $v_{1}$ and so $v_{2}$ centralizes $v_{1}$, whence $v_{1} \in T$ and $v \in P$. Again as $C_{Q}(a)=T$ for $a$ in $R^{\#}$, we see that all involutions of $S$ lie in either $Q$ or $P=T R$. Hence $Q$ and $P$ are the only elements of $N(S)$, which implies at once that each is normal in $N_{G}(S)$.

Again the conjugacy of $u$ and $v$ can be taken in $N=N_{G}(D)$ for some $D$ in $D$. Since $N=N^{*} N_{N}(S)$, where $N^{*}=O^{2}(N)$, we can therefore assume, in view of the preceding paragraph, that the conjugacy occurs in $N^{*}$. Again we set $Z=\Omega_{1}(Z(D))$. Suppose first that $v \in Z$. Since $v \in P-T=T R-T$, $C_{S}(v)=P$ and so $D \subseteq P$. But then $u \in Q \cap D=T$. However, as $v \in T R-$ $T, v$ is noncentral and hence so also is $u$, contrary to the fact that $T=Z(S)$.

So assume finally that $v \notin Z$, in which case also $u \notin Z$, whence $u \in Q-$ T. Then $C_{P}(u)=T$ and as $C_{Q}(v)=T$ and $Z$ centralizes both $u$ and $v$, we conclude that $Z=T$. Since $S$ centralizes $T=Z(S)$ and $N^{*}$ is generated by the conjugates of $S, N^{*}$ thus acts trivially on $Z$. But $N^{*}$ also acts trivially on 
$D / Z$ by (2.65) and consequently $N^{*}$ leaves both $Q \cap D$ and $P \cap D$ invariant, contrary to the fact that $u \sim v$ in $N^{*}$ and $u \in Q \cap D, v \in(P \cap D)-Q$.

We have therefore shown that $C_{P}(T) \nsubseteq T R$. Applying (2.24), we obtain

Lemma 5.9. $K$ is of type $A_{7}$ or $L_{2}\left(q^{2}\right), q \equiv 3,5(\bmod 8), q \geqslant 3$.

In particular, $T \cong D_{8}$ and $P / R \cong D_{8} \times E_{2}$. We can therefore write $P=$ $T \times R_{1}$, where $R_{1}$ contains $R$ as a subgroup of index 2. Since $R \subseteq Z(P)$, it follows that also $R_{1} \subseteq Z(P)$. Furthermore, except when $K$ is of type $L_{2}(9)$, we can choose $R_{1}$ so that $\Lambda\left(C_{K}\left(R_{1}\right)\right) \neq 1$.

We first prove

LEMMA 5.10. The following conditions hold:

(i) $R_{1} \cap Z(S) \neq 1$;

(ii) $R_{1}$ is elementary;

(iii) $K$ is of type $L_{2}(9)$;

(iv) $m(R) \leqslant 2$.

Proof. Suppose (i) is false. Since $T^{\prime} \subseteq Z(S) \subseteq Z(P)=T^{\prime} \times R_{1}$, our assumption implies that $T^{\prime}=Z(S) \cong E_{2}$. But as $P \triangleleft S$, also $Z(P) \triangleleft S$. This forces $R_{1}$ to be elementary, otherwise $\mho^{1}\left(R_{1}\right)=\mho^{1}(Z(P)) \triangleleft S$, whence $R_{1}$ $\cap Z(S) \neq 1$. Furthermore, as $[S, x] \subseteq Z(S) \cong E_{2},|S: P|=2$, so $S=P\langle w\rangle$ with $w^{2} \in P$. But $R_{1} \supset R$, so $m\left(R_{1}\right) \geqslant 2$. Considering the action of $w$ on $Z(P)=T^{\prime} \times R_{1}$, it follows that $C_{R_{1}}(w) \neq 1$. However, $C_{R_{1}}(w)$ centralizes $P\langle w\rangle=S$ and so $C_{R_{1}}(w) \subseteq Z(S)$, proving (i).

If $R_{1}$ is not elementary, then $\mho^{1}\left(R_{1}\right) \subseteq R$, whence $R \cap Z(S) \neq 1$ by (2.1), contrary to Lemma 5.4(i). Thus (ii) also holds.

Suppose next that $K$ is not of type $L_{2}(9)$, in which case $L_{2}\left(C_{K}(z)\right) \neq 1$ for $z$ in $R_{1}^{\#} \cap Z(S)$ and we are in case (i). We have $L_{x} \neq 1$ by $L$-balance, and since $S \subseteq C_{G}(z)$, our maximal choice of $P$ and $x$ is contradicted. Thus (iii) also holds.

Finally suppose that $m(R) \geqslant 3$. Since $Z(P)=T^{\prime} \times R_{1}$, we have $|Z(P): R|=4$. But then if we take $w$ in $S-P, w$ normalizes $Z(P)$ and so $R \cap R^{w} \neq 1$, contrary to Lemma 5.4(iii). Thus $m(R) \leqslant 2$, proving (iv).

We next prove

LeMma 5.11. $R_{1}$ is normal in $S$.

Proof. Suppose false. We have $R_{1}=\langle z\rangle \times R$, where $z \in Z(S)$ by the preceding lemma. Since $T^{\prime} \times R_{1}=Z(P) \triangleleft S$, it follows that for some $w$ in $S-P$ and $a$ in $R^{\#}, a^{w} \in Z(P)-R_{1}$. As usual, our maximal choice of $P$ implies that $P \in S\left(C_{G}(a)\right)$. 
Choose $B$ in $M(P)$. Then as $Z(P)$ is elementary, $B=B_{1} \times R_{1}$, where $B_{1}=B \cap T \cong E_{4}$. Moreover, $B \triangleleft S$. By the structure of $K, K$ contains a cyclic 3-subgroup $F$ which centralizes $R_{1}$ and acts nontrivially on $B_{1}$. Set $N=\langle F, S\rangle$, so that $N$ normalizes $B$ and centralizes $z$. We put $\bar{N}=N /\langle z\rangle$ and study the $\bar{N}$-conjugates of $\bar{a}$.

Since $T^{\prime} \subseteq B_{1}$, the involutions of $B_{1}$ are all central and hence so are those of $B_{1}\langle z\rangle$. Since $a \in R^{\#}$ is noncentral, $a$ is not conjugate to an element of $B_{1}\langle z\rangle$ and so all $\bar{N}$-conjugates of $\bar{a}$ lie in $\bar{B}-\bar{B}_{1}$. If $|R|=2$, our conditions imply that all elements of $\bar{B}-\bar{B}_{1}$ are conjugate, so $\bar{a}$ has 4 conjugates in $\bar{N}$ and consequently $\left|\bar{S}: C_{\bar{s}}(\bar{a})\right|=4$. But $\left|\bar{S}: C_{\bar{S}}(\bar{a})\right|=|[\bar{S}, \bar{a}]|=$ $\mid[\overline{S, a]}|\subseteq| \overline{Z(S)} \mid=2$. Since $m(R) \leqslant 2$ by the preceding lemma, we must have $R \cong E_{4}$. Moreover, we reach the same contradiction if the number of conjugates of $\bar{a}$ in $\bar{N}$ is a multiple of 4 .

We claim that $\bar{w}$ must centralize some $\bar{u}$ in $\bar{R}^{\#}$. Indeed, as $S^{\prime} \subseteq$ $B_{1}\langle z\rangle, S$ leaves $B_{1}\langle z\rangle$ invariant and consequently $\bar{N}$ normalizes $\bar{B}_{1}$. Hence the elements of $\bar{R}^{\#}$ are conjugate in $\bar{N}$ only to elements of $\bar{B}-\bar{B}_{1}$. Furthermore, the orbits of $\bar{F}$ on $\bar{B}-\bar{R}$ each contain 3 elements. But then if $\bar{R} \cap$ $\bar{R}^{\bar{w}}=1$, it follows easily as in the proof of Lemma 5.6 that the $\bar{N}$-classes of $\bar{B}-\bar{B}_{1}$ are unions of sets containing 4 elements and so the orbit of $\bar{a}$ has order divisible by 4 , which is not the case. Hence $\bar{R} \cap \bar{R}^{\bar{w}} \neq 1$ and so there exists $\bar{u}$ in $\bar{R}^{\#}$ such that $\bar{u}^{\bar{w}}=\bar{v} \in \bar{R}^{\#}$. If $\bar{v} \neq \bar{u}$, then $[\bar{u}, \bar{v}] \neq 1$ and so $\bar{S}^{\prime} \cap \bar{R} \neq 1$. But as $z$ and $S^{\prime}$ lie in $Z(S)$, this implies that $Z(S) \cap R \neq 1$, which is not the case. Thus $\bar{v}=\bar{u}$ and so $\bar{w}$ centralizes $\bar{u}$.

We now see that $\bar{R}=\langle\bar{a}, \bar{u}\rangle$ and that $\bar{B}-\bar{B}_{1}$ is the union of the sets $\{\bar{a}\} \cup \bar{B}_{1}^{\#} \bar{a}^{\bar{w}},\{\overline{a u}\} \cup \bar{B}_{1}^{\#}(\overline{a u})^{w}, \bar{B}_{1}^{\#} \bar{u}$, and $\{\bar{u}\}$ of orders $4,4,3,1$ respectively. All elements of each set are conjugate in $\bar{N}$. The orbit of $\bar{a}$ in $\bar{N}$ is the union of certain of these sets and so the number of conjugates of $\bar{a}$ in $\bar{N} \equiv 0,1,3(\bmod 4)$. However, this is impossible as the number of such conjugates $\equiv 2(\bmod 4)$ as $\left|\bar{S}: C_{\bar{S}}(\bar{a})\right|=2$.

As an immediate corollary we obtain

LemMa 5.12. We have $|R|=2$ and $|S|=64$.

Proof. We know that $\left|R_{1}: R\right|=2$ and that $C_{S}(a)=P$ for $a$ in $R^{\#}$. Since $R_{1} \triangleleft S$ and $S \supset P$, these conditions force $|R|=2$ and $\left|S / C_{S}\left(R_{1}\right)\right|=|S / P|=2$. But $T R$ has index 2 in $P$ and so $|S|=64$.

We next prove

LEMmA 5.13. We have $T \triangleleft S$.

Proof. Suppose false in which case some four subgroup $B_{1}$ of $T$ is not 
normal in $S$. Let $B=B_{1} \times R_{1}, F, N$, and $z$ be as in Lemma 5.11. As before, $F$ acts transitively on the sets $B_{1}^{\#} a, a \in R_{1}$, and there exists $w$ in $S-P$ which centralizes $z$ and interchanges $x$ and $x z .\left(R_{1}=\langle z, x\rangle\right.$ as $|R|=2$.) Furthermore, if $y \in B^{\#} \cap Z(S)$ we have that $(y x)^{w}=y x z$. We conclude from this that the following are subsets of the $N$-classes of $B^{\#}: B_{1}^{\#}, B_{1}^{\#} z,\{z\}$, $B_{1}^{\#}\{x, x z\}$, and $\{x, x z\}$.

If $b \in B_{1}^{\#}$, then $b$ is a central involution of $N$ and so $b$ cannot be fused to $x$ or $x z$. For the same reason the $N$-class $N_{b}$ of $b$ has odd order. Now $N_{b}$ is a union of $B_{1}^{\#}$ and at least one of the sets $B_{1}^{\#} z,\{z\}$, and $B_{1}^{\#}\{x, z\}$, as $B_{1}$ is not normal in $S$. The oddness of $\left|N_{b}\right|$ now forces $b$ to be fused to both $B_{1}^{\#} z$ and $\{z\}$ or to $B_{1}^{* \#}\{x, z\}$. We conclude that $\left|N_{b}\right|=$ 7,9 or 13 .

Since $\bar{N}=N / C_{N}(B)$ is isomorphic to a subgroup of $G L(4,2)$, whose order is not divisible by 13 , the third possibility is excluded. If $\left|N_{b}\right|=9$, then $N$ has Sylow 3-subgroups of order 9 and consequently $z$ would not be isolated in $N$. But in this case, the $N$-orbit $N_{z}$ of $z$ is a union of certain of the sets $\{z\}, B_{1}^{\#} z$, and $\{x, x z\}$. However, as $z$ is a central involution, the third set is excluded and so $\left|N_{z}\right|=4$, contrary to the fact that $z \in Z(S)$. Finally if $\left|N_{b}\right|=7$, then $N_{b}=\left\langle B_{1}, z\right\rangle^{\#}$ and $N$ contains a cyclic 7-subgroup $F_{1}$ which acts transitively on $\left\langle B_{1}, z\right\rangle^{\#}$. Then the orbits of $F_{1}$ on $B-\langle B, z\rangle$ have sizes 1 and 7 and consequently the sets $B_{1}^{\#}\{x, x z\}$ and $\{x, x z\}$ must be fused in $N$. Thus $x$ has 8 conjugates in $N$, contrary to the fact that $\left|S: C_{S}(x)\right|=|S: P|$ $=2$.

Now we can at last complete the proof of Proposition 5.2. Since $T \triangleleft S$ with $T \cong D_{8}$ and $S$ of class 2 , it follows that $S=T Q$, where $Q=C_{S}(T)$. Then $Q$ contains $T^{\prime} R_{1}$ as a subgroup of index 2. If $Q-T^{\prime} R_{1}$ contains an element $u$ with $u^{2} \in R_{1}$, then $S=T \times R_{1}\langle u\rangle$. Furthermore, as $C_{S}(x)=P$, $u$ does not centralize $x$ and so $R_{1}\langle u\rangle \cong D_{8}$. Thus $S \cong D_{8} \times D_{8}$, contrary to (2.74). Therefore no such $u$ exists.

Hence if $u \in Q-T^{\prime} R_{1}$, then $u^{2} \notin R_{1}$. Since $u^{2} \in Z(S)$, it follows that $u^{2}=t a$ with $\langle t\rangle=T^{\prime}$ and $a \in\langle z\rangle$. If $a=z$, then $(u x)^{2}=t z[u, x]=t z^{2}=t$. Hence replacing $u$ by $u x$, if necessary, we can suppose that $u^{2}=t$. Furthermore, if $t_{1}$ is an element of $T$ of order 4, we have $t_{1}^{2}=t$ and $t_{1}$ centralizes $u$, whence $t_{1} u$ is an involution of $S-P$. In addition, $C_{S}\left(t_{1} u\right)=\left\langle t_{1}\right\rangle \times\langle z\rangle \times\left\langle t_{1} u\right\rangle \cong Z_{4} \times E_{4}$. We easily compute now from the structure of $S$ that $C_{S}(w) \cong Z_{4} \times E_{4}$ for every $w$ in $I(S-P)$.

Observe next that $P=\Omega_{1}(P)$ and for any involution $v$ of $P$, we have $m\left(C_{P}(v)\right)=4$. It follows therefore from the preceding paragraph that $P \triangleleft N_{G}(S)$.

Finally we apply Thompson's fusion lemma. Taking $w$ in $I(S-P)$, we have that $w \sim v$ in $G$ for some $v$ in $P$. As usual, we can assume this 
conjugacy occurs in $N=N_{G}(D)$ for some $D$ in $D$. Since $N=N^{*} N_{N}(S)$, where $N^{*}=O^{2}(N)$, and $P \triangleleft N_{G}(S)$, we can suppose, in fact, that it takes place in $N^{*}$. Setting $Z=\Omega_{1}(Z(D))$, we have that $N^{*}$ acts trivially on $D / Z$. But then if $Z \subseteq P$, it would follow that $N^{*}$ normalized $P \cap D$, contrary to the fact that $v \in P \cap D$ and $w \in D-P$. Thus $Z \notin P$. Taking $w_{1}$ in $Z-$ $(P \cap Z)$, we have that $D \subseteq C_{S}\left(w_{1}\right) \cong Z_{4} \times E_{4}$, which implies that $|S / D| \geqslant 4$ and $m(Z) \leqslant 3$. However, this is impossible by (2.71) and Proposition 5.2 is proved.

With the aid of Proposition 5.2(i), we shall next prove

Proposition 5.14. There exists an $x$ in $I(G)$ such that $m\left(C_{G}(x)\right) \geqslant 5$ and $L_{\boldsymbol{x}} \neq 1$. In particular, $m(G) \geqslant 5$.

By Proposition 5.2(i), there is a central involution $z$ in $G$ for which $L_{z} \neq 1$. We choose $z$ so that $\left|L_{z} / O\left(L_{z}\right)\right|$ is maximal. The proposition holds if $m\left(C_{G}(z)\right) \geqslant 5$ and so we can assume that $m\left(C_{G}(z)\right) \leqslant 4$. As before, we shall derive a contradiction from this assumption.

We let $S$ be a Sylow 2-subgroup of $C_{G}(z)$, so that $S \in S(G)$. We again set $K=L_{z}, T=S \cap K$, and $R=C_{S}(K / O(K))$. Since $m\left(C_{G}(z)\right) \leqslant 4$ and no component of $K$ is of type $S L(2, q), q$ odd, it follows at once that $K$ consists of a single component of type $L_{2}(q), q$ odd, $A_{7}, U_{3}(4), J_{1}, L_{2}(8), U_{3}(8)$, $S z(8)$, or of Ree type. In particular, $T R=T \times R$. Furthermore, $m(R) \leqslant 2$ with $m(R)=1$ if $m(T)=3$.

We first prove

\section{LEMma 5.15. We have $S \supset T R$.}

Proof. Suppose by way of contradiction that $S=T R=T \times R$. We note that our argument will use only the fact that $S$ has the form $T \times R$ with certain possibilities for $T$ and will depend on no other properties of $T$ and $R$. Consider first the case that $m\left(R^{\prime}\right)=2$. By Goldschmidt's theorem, some $y \in$ $R^{\prime}$ has a conjugate in $S-R^{\prime}$. As usual, the fusion can be taken in $N=N_{G}(D)$ for some $D$ in $D$. Set $\bar{N}=N / D C_{N}(D)$. If $\bar{R}=1$, then $R \subseteq D$ and so $D=R \times(D \cap T)$. By the Krull-Schmidt theorem, either $R^{\prime} \triangleleft N$ or $D \cap T$ has a subgroup isomorphic to $R$. However, as $y \in R^{\prime}$ is fused in $N$ to an element of $D-R^{\prime}$, the second alternative must occur. Since $m(R)=2$ in this case, $m(T)=2$ and so $T$ is dihedral. But as $R$ is isomorphic to a subgroup of $T$, this forces $m\left(R^{\prime}\right) \leqslant 1$, contrary to $m\left(R^{\prime}\right)=2$. Thus $\bar{R} \neq 1$.

By the structure of $\bar{N}$, the conjugates of $\bar{R}$ in $\bar{N}$ generate $\bar{N}^{*}=$ $O^{2^{\prime}}(\bar{N})$. But as $R^{\prime} \subseteq Z(R)$ and $m\left(R^{\prime}\right)=m(R)=2, R^{\prime}=\Omega_{1}(R)$ whence $\Omega_{1}(S)=\Omega_{1}(T) \times \overline{R^{\prime}}$. Hence $R$ centralizes $\Omega_{1}(S)$ and so centralizes $Z=$ $\Omega_{1}(Z(D))$. Thus $\bar{N}^{*}$ does as well. But $y \in Z(S)$ as $y \in R^{\prime} \subseteq Z(S)$ and 
$\bar{N}=\bar{N}^{*} N_{\bar{N}}(\bar{S})$. Hence the fusion can be assumed to take place in $N_{G}(S)$ and so we can suppose that $S=D$. But then $\bar{R}=1$, contrary to the preceding paragraph. We therefore conclude that $m\left(R^{\prime}\right) \leqslant 1$.

If $T$ is of type $S z(8), U_{3}(4)$, or $U_{3}(8)$, we can repeat the preceding argument with $T$ and $R$ interchanged. With the corresponding $D, N$, and $\bar{N}$, if $\bar{T}=1$, we obtain this time that $D=T \times D \cap R$. Likewise the Krull-Schmidt theorem and the fact that some involution of $T^{\prime}$ is fused in $N$ to an element of $D-T^{\prime}$ implies that $D \cap R$ contains a subgroup isomorphic to $T$. But then as $m\left(T^{\prime}\right) \geqslant 2$, also $m\left(R^{\prime}\right) \geqslant 2$, contrary to what we have just shown. Hence $\bar{T} \neq 1$ and, as $T^{\prime}=\Omega_{1}(T)$, we can reduce, as above, to the case $S=D$, contrary to $\bar{T} \neq 1$. Therefore $T \cong E_{4}, E_{8}$, or $D_{8}$.

Next assume $m\left(R^{\prime}\right)=1$, let $y$ be the involution of $R^{\prime}$, and again consider $D, N$, and $\bar{N}$. If $\bar{R}=1$, we again have $D=R \times(D \cap T)$ and $D \cap T$ contains a subgroup isomorphic to $R$. Since $R$ is nonabelian, this forces $R \cong$ $T \cong D_{8}$, so $S \cong D_{8} \times D_{8}$, contrary to (2.74). Thus $\bar{R} \neq 1$. If $N^{*}=O^{2^{\prime}}(N)$ centralizes $y$, we can again reduce to the case $S=D$, contrary to $\bar{R} \neq 1$. Hence we can also assume that $\bar{N}^{*}$ does not centralize $y$. But then (2.65) yields that $y \notin D^{\prime}$ or $\mho^{1}(D)$. Since $R^{\prime}$ is cyclic, the first condition implies that $R \cap D$ is abelian and now the second yields that $R \cap D=\langle y\rangle \times B$ for some cyclic subgroup $B$.

Suppose now that $T \subseteq D$. Since $R$ centralizes $T$ and $C_{S}(D) \subseteq D$, we have $C_{R}(R \cap D) \subseteq R \cap D$. Since $R \nsubseteq D, R \cap D \nsubseteq Z(R)$ and, in particular, $B \neq 1$. If $R \cap D \cong E_{4}$, then necessarily $R \cong D_{8}$. Since $m(S) \leqslant 4, T \nRightarrow E_{8}$ and so $T \cong E_{4}$ or $D_{8}$, whence $S \cong E_{4} \times D_{8}$ or $D_{8} \times D_{8}$, again contradicting (2.74). Thus $|B|>2$. In this case, $\Omega_{1}(B) \subseteq Z(S) \cap R \subseteq Z(R)$. Since also $y \in Z(R)$, it follows that $\Omega_{1}(R \cap D) \subseteq Z(R)$. This implies that $R$ centralizes $\Omega_{1}(D)$ and hence so does $\bar{N}^{*}$. Once again we can now reduce to the case $S=$ $D$, contrary to $\bar{R} \neq 1$. We have therefore shown that $T \notin D$.

If $T$ were abelian, then $T \subseteq Z(S) \subseteq D$, so $T$ is nonabelian and hence $T \cong D_{8}$. If $D$ covers $T R / R$, then $m(Z(D)) \leqslant 3$. Hence by $(2.71),|S: D|=2$. This in turn implies that $D$ also covers $T R / T$. Since $T R=T \times R$, it follows that $Z(D)=Z(S)$. But then $\bar{N}^{*}$ centralizes $Z(D)$ and so centralizes $y$, which is not the case. On the other hand, if $|D \cap T| \leqslant 2$, then $|S: D| \geqslant 4$, while $m(Z(D)) \leqslant 3$, contrary to (2.71). Thus $|D \cap T|=4$ and $D$ does not cover $(T R) / R$, which implies that $D=(D \cap T) \times(D \cap R)$. Again we have $|S: D|$ $\geqslant 4$ and now (2.71) implies that $\bar{N}^{*} \cong L_{2}(4)$ and that $D \cong E_{16}$. Thus $D \cap$ $T \cong D \cap R \cong E_{4}$, whence $R \cong D_{8}$ and again $S \cong D_{8} \times D_{8}$, contradicting (2.74). We have therefore finally eliminated the case $m\left(R^{\prime}\right)=1$ and have therefore shown that $R$ is abelian.

But now $R \subseteq Z(S)$ as $S=T \times R$. Since $Z(S)$ is elementary by (2.1), it 
follows that $R \cong E_{2}$ or $E_{4}$. But $S$ is nonabelian and so $T \cong D_{8}$, whence $S=$ $D_{8} \times E_{2}$ or $D_{8} \times E_{4}$. In either case, (2.74) yields a contradiction.

Since we know the possibilities for $K$ and since $S \neq T R,(2.18)$ now yields

Lemma 5.16. $K$ is of type $L_{2}(q), q$ odd, or $A_{7}$. In particular, $T \cong E_{4}$ or $D_{8}$.

As remarked above the argument of Lemma 5.15 applies equally well in the case $S=T_{1} \times R_{1}$ with $T_{1} \cong E_{4}$ or $D_{8}$ to yield the same contradiction. Hence we also have

Lemma 5.17. $S$ is not of the form $T_{1} \times R_{1}$ with $T_{1} \cong E_{4}$ or $D_{8}$. As a corollary, we obtain

LEMMA 5.18. We have $T \cong E_{4}$.

Proof. If false, then $T \cong D_{8}$ and by (2.18), $S / R \cong D_{8} \times E_{2}$ and $S / R=$ $T R / R \times R_{1} / R$ for some subgroup $R_{1}$ of $S$ with $\left|R_{1}: R\right|=2$. Thus $S=$ $T \times R_{1}$, contrary to the preceding lemma.

We next prove

LEMMA 5.19. We have $\Omega_{1}(R)$ is abelian.

Proof. Suppose false, in which case $R$ contains two noncommuting involutions. Since $R$ has class at most 2 , the dihedral group $~ V$ which they generate is necessarily isomorphic to $D_{8}$. Since $m(R) \leqslant 2$ and $R \triangleleft S$, either $Z(S) \cap$ $R \cong Z_{2}$ or $E_{4}$. However, in the latter case clearly $Z(S) \cap R=\Omega_{1}(R)$ and $\Omega_{1}(R)$ is abelian, contrary to assumption. We thus conclude that $Z(S) \cap R=$ $\langle z\rangle=V^{\prime}$. Since $S$ has class 2 and $R \triangleleft S$, this in turn implies that $V \triangleleft S$ and that $S$ centralizes $V / V^{\prime}$. It follows therefore that $S=V C_{S}(V)$.

Since $S / R \cong D_{8}, S-T R$ contains an element whose square lies in $R$ and so $S=T R_{1}$ for some subgroup $R_{1}$ with $T \cap R_{1}=1$ and $R_{1}$ containing $R$ as a subgroup of index 2. Setting $Q=C_{R}(V)$ and $Q_{1}=C_{R_{1}}(V)$, we have that $R=V Q, R_{1}=V Q_{1}$, and $Q_{1}$ contains $Q$ as a subgroup of index 2. Furthermore, as $m(R)=2, Q \cong Z_{2}, Z_{4}$, or $Q_{8}$. Observe next that if $Q_{1}=$ $Q \times Y$ with $Y \cong Z_{2}$, then $S=T Y \times V Q$ with $T Y \cong D_{8}$ and we see that Lemma 5.17 is contradicted. Hence $Q_{1}$ is not of this form and as $R_{1}$ has class 2 and exponent 4 , we conclude now that $Q_{1} \cong Z_{4}, D_{8}, Q_{8}$, or $Q_{8} * Z_{4}$.

We have that $Z(S)=\langle t, z\rangle$, where $\langle t\rangle=T \cap Z(S)$, and we argue next that no two of the involutions $z, t, t z$ are conjugate in $G$. Indeed, if false, then by a result of Burnside, this conjugation would occur in $N_{G}(S)$ and so $N_{G}(S)$ 
would necessarily contain a 3-element which cyclically permuted the involutions of $Z(S)$. But then $S /\langle t\rangle \cong S /\langle z\rangle$. However, as $S=T V Q_{1}$, we see that $S /\langle z\rangle$ $\cong D_{8} \times E_{2^{n}}$ for some $n$ and so $S /\langle z\rangle$ does not possess a subgroup isomorphic to $V Q_{1}$. On the other hand, clearly $S /\langle t\rangle$ does possess such a subgroup. This contradiction establishes our assertion.

Now we can complete the proof. We know that $z$ is not isolated in $G$ and so is not isolated in $N=N_{G}(D)$ for some element $D$ in $D$. Since $N=$ $N^{*} N_{N}(S)$, where $N^{*}=O^{2^{\prime}}(N)$, and since $z$ is isolated in $N_{G}(S)$ by the preceding paragraph, we have that $z$ is not isolated in $N^{*}$. It follows therefore from (2.65) that $z \notin \mho^{1}(D)$. But $R_{1}=V Q_{1} \cong D_{8} * Z_{4}, D_{8} * D_{8}, D_{8} * Q_{8}$, or $D_{8} * Q_{8} * Z_{4}$. Hence if $R_{0}$ is a subgroup of index at most 2 in $R_{1}$, we see in each case that $z \in \mho^{1}\left(R_{0}\right)$. Thus no such subgroup $R_{0}$ can lie in $D$ and consequently $\left|R_{1} / R_{1} \cap D\right| \geqslant 4$, whence $|S / D| \geqslant 4$. On the other hand, as $m(S) \leqslant$ 4, we conclude now from (2.71) that $Z=\Omega_{1}(Z(D)) \cong E_{16}$ and that $N^{*}$ acts transitively on $Z^{\#}$. Since $Z(S) \subseteq Z$, all involutions of $Z(S)$ are thus conjugate in $G$, contrary to what we have shown above.

We can now prove

LEMMA 5.20. $S-T R$ contains an involution.

Proof. Suppose false, in which case $B=\Omega_{1}(S)=T \times \Omega_{1}(R)$. By the preceding lemma, we have that $B$ is elementary. Clearly $B$ is strongly closed in $S$, so Proposition 3.1 is contradicted.

We let $y$ denote an arbitrary involution of $S-T R$. Then $T\langle y\rangle \cong D_{8}$. We also set $B=\Omega_{1}(T R)=T \times \Omega_{1}(R)$, so that $B$ is elementary. We fix this notation. To analyze the remaining situation, we need the following preliminary result.

LEMMA 5.21. If $m(Z(S))=2$, then $z$ is not conjugate in $G$ to an involution of $T R-\langle z\rangle$.

Proof. Suppose false, so that $z \sim u$ in $G$ for some $u \neq z$ in $B$. If $B$ is weakly closed in $S$, then $z \sim u$ in $N=N_{G}(B)$. On the other hand, by the structure of $K, S$ is permutable with a cyclic 3-subgroup $F$ of $K$ with $F$ centralizing $R$ and acting nontrivially on $T$. Since $T \subseteq B, F$ normalizes $B$ and so $F \subseteq N$. Moreover, if we put $P=T R$, it follows again from the structure of $K$ that $C_{S}(B) \subseteq P$ and that $P=O_{2}(S F)$. Since $T=[P, F] \cong E_{4}$ and $R$ centralizes $F$, we conclude that all the assumptions of (2.68) are satisfied with $N$ in place of $H$. Since $m(Z(S))=2$, we conclude that $S \cong D_{8} \times D_{8}$ or $E_{4}, E_{2}$, contrary to (2.74). Hence $B$ is not weakly closed in $S$ and $B \neq B^{g} \subseteq S$ for some $g \in G$. Since $y$ does not centralize $T$, it follows that $B \in M(S)$. Thus $B^{g} \in$ 
$M(S)$ whence $B^{g} \triangleleft S$. A result of Burnside implies that we may assume $g$ lies in $N=N_{G}(S)$. As $B \triangleleft S$, we may take $g$ to be of odd order. If $z^{g}=z$, then $g \in M=C_{G}(z)$. But by the structure of $M, M$ has a subgroup of index 2 with $T R$ as Sylow 2-subgroup. Hence $g$ normalizes $T R$ and so $B^{g} \subseteq \Omega_{1}(T R)=B$, which is not the case. Therefore we also have that $z^{g} \neq z$.

Under the present assumptions, clearly $Z(S)=\langle t, z\rangle$, where $\langle t\rangle=T \cap$ $Z(S) \cong E_{2}$. Hence $\langle z\rangle=R \cap Z(S)$ and so also $\left\langle z^{g}\right\rangle=R^{g} \cap Z(S)$. Since $R$ and $R^{g}$ are each normal in $S$, the assumption $R \cap R^{g} \neq 1$ would imply that $R \cap R^{g} \cap Z(S) \neq 1$, whence $z=z^{g}$, which is not the case. Hence we must have $R^{g} \cap R=1$. But $S / R \cong D_{8}$, so $R^{g}$ and hence also $R$ is isomorphic to a subgroup of $D_{8}$. As $\Omega_{1}(R)$ is abelian, we have, in fact, $R \cong E_{2}, E_{4}$, or $Z_{4}$. However, the first case is excluded as then $S=T\langle y\rangle \times R$, contrary to Lemma 5.17. For the same reason, $y$ does not centralize $R$. But then if $R \cong E_{4}$, we see that $S=E_{4} \sim E_{2}$, contrary to (2.74). On the other hand, if $R \cong Z_{4}$, we check directly that $S=(T \times R)\langle y\rangle$ possesses no nontrivial automorphisms of odd order. Since $|g|$ is odd and $g$ normalizes $S, g$ thus centralizes $S$, whence $z^{g}=z$, which is not the case. Thus our initial supposition leads to a contradiction, and the lemma is established.

We can now prove

\section{LEMmA 5.22. $y$ is not extremal in $S$.}

PROof. Suppose by way of contradiction that $y$ is extremal in $S$. By Thompson's fusion lemma, we have $u^{g}=y$ and $C_{S}(u)^{g} \subseteq C_{S}(y)$ for some $u$ in $T R$ and $g$ in $G$.

Now $C_{T}(y)=\langle t\rangle \cong Z_{2}$ and $C_{S}(y)=\langle t, y\rangle \times C_{R}(y) \cong E_{4} \times C_{R}(y)$. On the other hand, $C_{S}(u) \supseteq T \times C_{R}(u)$. Moreover, if $u=t a, t \in T, a \in R$, we have $C_{R}(u)=C_{R}(a)$. But then if $a \in Z(R)$, we have $T \times R \subseteq C_{S}(u)$ whence $(T \times R)^{g} \subseteq C_{S}(y)=\langle t, y\rangle \times C_{R}(y)$ forcing $\left|C_{R}(y)\right|=|R|$. Thus $y$ centralizes $R$ and so $S=T\langle y\rangle \times R$ contrary to Lemma 5.17. Hence $a \notin$ $Z(R)$. Since $m(R)=1$ or 2 , this forces $m(Z(R))=1$, whence $z$ is the unique involution of $R \cap Z(S)$. Thus $Z(S)=\langle t, z\rangle$ and we conclude that $m(Z(S))=2$. Hence the preceding lemma is applicable.

Suppose $z \in \mho^{1}\left(C_{S}(u)\right)$ or $C_{S}(u)^{\prime}$. Since $|S: T R|=2$, it follows then that $z^{g} \in T R$. Now the preceding lemma yields $z^{g}=z$, whence $g \in M=$ $C_{G}(z)$. But $M$ has a normal subgroup of index 2 with Sylow 2-subgroup $T R$. Hence $y$ and $u$ cannot be conjugate in $M$. This contradiction shows that $z \notin \mho^{1}\left(C_{S}(u)\right)$ or $C_{S}(u)^{\prime}$.

Since $\Omega_{1}(R)$ is abelian and $m(R) \leqslant 2$, we see that $\Omega_{1}(R)=\langle a, z\rangle$. Thus $\langle a, z\rangle \triangleleft R$ and so $R_{0}=C_{R}(a)$ has index at most 2 in $R$. We have $z \notin$ 
$R_{0}^{\prime}$ or $\mho^{1}\left(R_{0}\right)$, so $R_{0}$ is abelian of type $\left(2^{n}, 2\right)$ for some $n$. If $n>1$, then $\mho^{1}\left(R_{0}\right)$ would contain a central involution of $R$, forcing $z \in \mho^{1}\left(R_{0}\right)$ as $\langle z\rangle=$ $\Omega_{1}(Z(R))$, a contradiction. Hence $n=1$ and so $|R| \leqslant 8$. Since $a \notin Z(R)$, we have $R \cong D_{8}$, contrary to the fact that $\Omega_{1}(R)=\langle a, z\rangle \cong E_{4}$. This completes the proof of the lemma.

As our final lemma, we have

LEMMA 5.23. The following conditions hold:

(i) $C_{S}(y)$ is abelian;

(ii) $m(Z(S))=2$.

PRoof. Choose $P$ in $S\left(C_{G}(y)\right)$ containing $C_{S}(y)$. We argue first that $Z(R) \cap Z(P)=1$. Indeed, suppose false and let $a \in I(Z(R) \cap Z(P))$. Since $y$ centralizes $a$, we have that $a \in Z(S)$. Hence $H=C_{G}(a)$ contains $S$ as well as $P$, so $P^{g} \subseteq S$ for some $g$ in $H$. Since $P^{g} \in S\left(C_{G}\left(y^{g}\right)\right)$, it follows therefore that $y^{g}$ is extremal in $S$. On the other hand, the preceding lemma applies to any involution of $S-T R$ and consequently to derive a contradiction, it will suffice to prove that $y^{g} \in S-T R$.

Set $J=L_{2}\left(C_{K}(a)\right)$, so that $J$ covers $K / O(K)$ and by $L$-balance, $J \subseteq L_{a}$. By our choice of $z$ and the fact that $a \in Z(S)$, we have $|K / O(K)| \geqslant\left|L_{a} / O\left(L_{a}\right)\right|$ and consequently $J$ covers $L_{a} / O\left(L_{a}\right)$. Hence if we set $\bar{H}=H / O(H)$, we have that $\bar{J}=\bar{L}_{a}=L(\bar{H})$ and we conclude at once that $\bar{T}=\bar{S} \cap \bar{L}_{a}$ and that $\bar{R}$ is a Sylow 2-subgroup of $C_{\bar{H}}\left(\bar{L}_{a}\right)$. Furthermore, considering the action of $y$ on $J$, we have that $\bar{L}_{a}\langle\bar{y}\rangle \cong P G L(2, q)$. Since $g \in H$ and $L_{a} \triangleleft H$, likewise $\bar{L}_{a}\left\langle\bar{y}^{g}\right\rangle \cong P G L(2, q)$, whence $\bar{y}^{g} \notin \bar{L}_{a} C_{\bar{H}}\left(\bar{L}_{a}\right)$. It follows at once that $y^{g} \in S-T R$, giving the desired contradiction.

Now we can establish the lemma. We have that $C_{S}(y)=\langle t, y\rangle \times Q$, where $\langle t\rangle=C_{T}(y)$ and $Q=C_{R}(y)$. Hence if $C_{S}(y)$ is nonabelian, then so is $Q$. But $Q^{\prime} \subseteq Z(R)$. Since $Q \subseteq P$, likewise $Q^{\prime} \subseteq Z(P)$. Thus $Q^{\prime} \subseteq Z(R) \cap Z(P)=1$, whence $Q$ is abelian. We conclude that $C_{S}(y)$ is abelian, proving (i).

By the structure of $S$, we have that $Z(S)=\langle t\rangle \times C_{Z(R)}(y)=\langle t\rangle \times(Z(S) \cap R)$. Since $z \in Z(S) \cap R$, either (ii) holds or $m(Z(S) \cap R) \geqslant 2$. Consider the latter possibility, whence $m(Z(S)) \geqslant 3$. If $S^{*}$ is a Sylow 2-subgroup of $G$ containing $P$, then $Z\left(S^{*}\right) \subseteq Z(P)$, so $m(Z(P)) \geqslant 3$. But $Z(S) \cap R \subseteq P$ and is noncyclic. Since $m(P) \leqslant 4$, it follows that $(Z(S) \cap R) \cap Z(P) \neq 1$. Since $Z(S) \cap R \subseteq Z(R)$, we conclude that $Z(R) \cap Z(P) \neq 1$, contrary to what we have shown above. Thus (ii) also holds.

We are at last in a position to complete the proof of Proposition 5.14. We assume $y$ is chosen in $S-T R$ so that $\left|C_{S}(y)\right|$ is maximal. We have $y^{g}=u$ for some $g$ in $G$ with $\left|C_{S}(u)\right|>\left|C_{S}(y)\right|$ as $y$ is not extremal in $S$. Clearly then 
$g$ cannot be taken in $N_{G}(S)$. As usual, we can now assume without loss that $g \in N^{*}=O^{2}\left(N_{G}(D)\right)$ for some $D$ in $D$. By our choice of $y$ we also have that $u \in T R$.

By (2.65), $N^{*}$ acts trivially on $D / Z(D)$, so $u=y^{g}=y w$ for some $w$ in $Z(D)$. Then $w$ is an involution and as $u \in T R$, it follows that $w \notin T R$. Lemma 5.23 applies to any involution of $S-T R$ and, in particular, to $w$. Thus $C_{S}(w)$ is abelian and contains $D$. But $C_{S}(D) \subseteq D$ and consequently $D=$ $C_{S}(w)$ and $D$ is abelian. Since $y \in D$, we have $T \cap D=C_{T}(y)=\langle t\rangle$. Furthermore, as $y$ centralizes no element of $T R-\langle t\rangle R$, it follows that $T R \cap$ $D=\langle t\rangle \times(D \cap R)$. If $R \subseteq D$, then $y$ centralizes $R$ and so $S=T\langle y\rangle \times R$, contrary to Lemma 5.17. Thus $R \nsubseteq D$ and we conclude now that $|T R: D| \geqslant$ 4, whence $|S: D| \geqslant 4$.

Finally applying (2.71), we have that $D \cong E_{16}$. Thus $D=\langle y, t\rangle \times$ $(D \cap R)$ with $U=D \cap R \cong E_{4}$. Furthermore, $|S: D|=4$ and by the above decomposition of $D$, we see that $|R|=8$. Since $y$ centralizes $U$, the assumption $R$ abelian would imply that $U \subseteq Z(S)$, whence $m(Z(S))=3$, contrary to Lemma 5.23. Thus $R \cong D_{8}$. Since $\bar{y}$ centralizes $U$, we conclude now that $R\langle y\rangle=R \times\left\langle y_{1}\right\rangle$ for some involution $y_{1}$. Hence $S=T\left\langle y_{1}\right\rangle \times R$, contrary to Lemma 5.17. This completes the proof of Proposition 5.14.

6. The odd $A$-hull. Our goal in the next two sections will be to prove that $L_{1}(G)$ is either empty or consists of an evenly embedded $L_{2}(5)$.

To this end, we define for any subgroup $Q$ of $G$

$$
V_{Q}^{*}=\left\langle\Lambda_{x}^{*} \mid x \in(Q)\right\rangle \text {. }
$$

We call $V_{Q}^{*}$ the odd $Q$-hull.

We shall here prove

Proposition 6.1. For any $A$ in $M(G)$, the odd $A$-hull $V_{A}^{*}$ is a proper subgroup of $G$.

Fix any $A$ in $M(G)$. By Proposition 5.14, we have $m(A) \geqslant 5$. First of all, if $\Lambda_{x}=1$ for all $x$ in $A^{\#}$, then by (2.50(ii)) $\Lambda_{x}^{*}=O\left(C_{G}(x)\right)$ for each $x$ in $A^{\#}$ and by (2.40) $G$ is 1 -balanced with respect to $A$. We can therefore apply (2.56) to conclude that

$$
V_{A}^{*}=\left\langle O\left(C_{G}(x)\right) \mid x \in A^{\#}\right\rangle
$$

has odd order. Since $|G|$ is even, clearly $V_{A}^{*} \subset G$ in this case.

We can therefore assume $\Lambda_{x} \neq 1$ for some $x$ in $A^{\#}$. We shall treat two possibilities by separate arguments: 
Core case. $\Lambda_{x}$ is not semisimple for some $x$ in $A^{\#}$. Semisimple case. $\Lambda_{x}$ is semisimple for all $x$ in $A^{\#}$.

We first consider the core case. We set

$$
W_{A}=\left\langle\Delta_{G}(T) \mid T \in E_{3}(A)\right\rangle .
$$

By (2.39), $G$ is 3-balanced with respect to $A$ and consequently (2.56) yields

Lemma 6.2. $W_{A}$ is of odd order.

Our goal now will be to prove that $W_{A}$ is nontrivial. Once this is accomplished we shall argue that $V_{A}^{*} \subseteq N_{G}\left(W_{A}\right)$ except in certain cases when $m(A)=5$.

First of all, we choose $x$ in $A^{\#}$ so that $\Lambda_{x}$ is not semisimple (as we may) and set $H=C_{G}(x)$ and $\bar{H}=H / O(H)$. By (2.10), we have $[K, O(H)] \neq 1$ for some component $K$ of $\Lambda_{x}$. Since $\bar{K} \# S L(2, q), q$ odd, $\bar{K} \in A_{1}$ and so if we set $A_{0}=C_{A}(\bar{K}),(2.37)$ implies that $m\left(A / A_{0}\right)=2$ or 3 . Moreover, in the latter case, it follows from (2.26) that there exists $a$ in $A-A_{0}$ such that $\Lambda\left(C_{\bar{K}}(a)\right) \neq 1$. For uniformity of notation, set $B=A_{0}$ if $m\left(A_{0}\right) \geqslant 4$, while if $m\left(A_{0}\right) \leqslant 3$, set $B=A_{0}$ or $B=A_{0}\langle a\rangle$ according as $m\left(A / A_{0}\right)=2$ or 3 . Furthermore, set $J=\Lambda\left(C_{K}(B)\right)$. Then $J$ maps onto $\bar{K}$ or onto $\Lambda\left(C_{\bar{K}}(a)\right)$ according as $B=A_{0}$ or $B=A_{0}\langle a\rangle$. In particular, $O(J) \subseteq O(H)$.

Since $K$ is perfect and $\bar{K}$ is simple, $C_{K}(O(H)) \subseteq O(K)$ and so as $K$ does not centralize $O(H)$, neither does $J$. Since $O(J \subseteq O(H)$, we can apply (2.55) to conclude that for some subgroup $E$ of index at most 2 in $B, J$ does not centralize

$$
D=\left[C_{O(H)}(E), J\right]
$$

We choose $E$ as large as possible so that $D \neq 1$. By (2.11) we have $[D, J]=D$.

We fix all this notation. The key step in the argument is the following

Lemma 6.3. We have $D \subseteq \Delta_{G}(E)$.

Proof. Indeed, let $t \in E^{\#}$. We must show that $D \subseteq O\left(C_{G}(t)\right)$. It is immediate from the action of $B$ and $E$ on $K$ that $J \subseteq \Lambda\left(C_{K}(t)\right)$ and $E \subseteq$ $A \in M(G) . \Lambda$-balance together with (2.43(iii)) implies that $\Lambda\left(C_{K}(t)\right)$ and hence $J$ lie in a unique component $L$ of $\Lambda_{t}$. Since $D$ centralizes $t$ and $[D, J]$ $=D$ and since $L \triangleleft \Lambda_{t}$, it follows that $D \subseteq L$. Set $\bar{L}=L / O(L)$, so that $\bar{L} \in A_{1}$. Since $A$ leaves $L$ invariant and $\bar{x}$ centralizes $J, C_{\bar{L}}(x)$ is nonsolvable and consequently $L\left(C_{\bar{L}}(x)\right) \neq 1$ and $O\left(C_{\bar{L}}(x)\right)=1$ by (2.28). On the other hand, as $D \subseteq O\left(C_{G}(x)\right)$ and $D \subseteq L, \bar{D} \subseteq O\left(C_{\bar{L}}(x)\right)$, so $\bar{D}=1$. Thus $D \subseteq O(L) \subseteq O\left(C_{G}(t)\right)$, as required. 
As a corollary, we have

LEMMA 6.4. Either $W_{A} \neq 1$ or $m(A)=5, m(E)=2, m(B)=3$, and $E=C_{B}(D)$.

Proof. If $m(E) \geqslant 3$, we choose $T$ in $E_{3}(E)$. Then as $\Delta_{G}(T) \supseteq$ $\Delta_{G}(E)$, we have $D \subseteq \Delta_{G}(T)$ by the preceding lemma and as $D \neq 1$, it follows that $\Delta_{G}(T) \neq 1$. Hence $W_{A} \neq 1$ in this case. On the other hand, if $m(E)<$ 3, we conclude at once from the definitions of $B$ and $E$ and the fact that $m(A) \geqslant 5$ that $m(A)=5, m(E)=2, m(B)=3$, and $E=C_{B}(D)$.

It thus remains to prove that $W_{A} \neq 1$ in this exceptional case. Since $J$ centralizes $B$ and $J \cap B=1, m(J)=2$ (as $m(A)=m(G)=5$ ). Let $U$ be a four subgroup of $J$ containing $A \cap J$ and set $A^{*}=U \times B$. Then $m\left(A^{*}\right)=5$ and so $A^{*} \in M(G)$. Furthermore, $m\left(A \cap A^{*}\right) \geqslant 4$ as $A \cap J \neq 1$. Hence if we define $W_{A}$ * by analogy with $W_{A}$, we have that $W_{A}=W_{A} *$ by (2.57). Thus it will suffice to prove that $W_{A} \neq 1$. Since $U \subseteq A^{*}$, we can therefore suppose without loss to begin with that $U \subseteq A$.

Since $J$ does not centralize $\bar{D}$, neither does $U$. Choose $u$ in $U^{\#}$. Since $N_{J}(U)$ acts transitively on $U^{\#}$, it follows that

$$
F=\left[C_{D}(u), U\right] \neq 1 \text {. }
$$

Note that $F$ is $A$-invariant and $F=[F, U]$. Also as $N_{J}(U)$ centralizes $B$, Lemma 6.4 implies that $E=C_{B}(F)$. We set $T=E\langle u\rangle$. Since $D$ centralizes $E, F$ centralizes $T$.

We first prove

Lemma 6.5. If $t \in T^{\#}$, then either $F \subseteq O\left(C_{G}(t)\right)$ or $t=u$ and $\left|F / F \cap O\left(C_{G}(u)\right)\right|=3$.

Proof. Set $N=C_{G}(t)$ and $\bar{N}=N / O(N)$. We can suppose that $F \nsubseteq$ $O(N)$ so that $\bar{F} \neq 1$. By Lemma 6.3, we have that $t \notin E$. Since $m(G)=5$, $L(\bar{N})$ consists of at most two components and so $\bar{F}$ leaves each component of $L(\bar{N})$ invariant. Since $\left|\left[F, C_{N}(x)\right]\right|$ is odd, $\bar{F}$ centralizes $C_{O_{2}(\bar{N})}(\bar{x})$ and so $\bar{F}$ centralizes $O_{2}(\bar{N})$ by Thompson's $A \times B$-lemma. Hence by $(2.8), \bar{F}$ does not centralize some component $\bar{X}$ of $L(\bar{N})$. Since $\bar{X}$ and $\bar{F}$ are both $\bar{A}$-invariant, $\bar{Y}=\overline{X F A}$ is a group. Setting $\widetilde{Y}=\bar{Y} / C_{\bar{Y}}(\bar{X})$, we have that $\widetilde{F} \neq 1$, and that $\tilde{Y}$ is isomorphic to a subgroup of $\operatorname{Aut}(\widetilde{X})$. Since $\widetilde{X} \in A, \tilde{Y} / \tilde{X}$ is abelian by (2.17). But $\widetilde{F}=[\widetilde{F}, \widetilde{U}]$, so $\widetilde{F} \subseteq \widetilde{X}$.

By Lemma 6.3, $F \subseteq O\left(C_{G}(y)\right)$ for all $y$ in $E^{\#}$ and so $\bar{F} \subseteq O\left(C_{\overline{X F}}(\bar{y})\right)$. Since $\widetilde{X} \cong \overline{X F} / O \overline{(X F)}$, it follows that $\widetilde{F} \subseteq O\left(C_{\widetilde{X}}(\tilde{y})\right)$. Hence $L\left(C_{\widetilde{X}}(\tilde{y})\right)=1$ for each $y$ in $E^{\#}$ by (2.28). In particular, $C_{\bar{E}}(\widetilde{X})=1$ and so $\underset{E}{\widetilde{X}}$ is a four 
group. Moreover, by (2.29(ii)). it also follows that $\bar{X} \cong L_{2}(q), q$ odd, or $A_{7}$. However, in the first case, $C_{\widetilde{X}}(\widetilde{E})$ is a 2-group by $(2.29(\mathrm{v}))$. Since $\widetilde{F} \subseteq \widetilde{X}$ centralizes $\widetilde{E}$, we conclude that $\widetilde{X} \cong A_{7}$, that $\widetilde{E}$ is a four subgroup of $\widetilde{X}$, and that $|\widetilde{F}|=3$. Furthermore, as $U \cap E=1$ and $U$ centralizes $E$, but does not centralize $\widetilde{F}$, we have that $\tilde{X} \widetilde{U} \cong \mathrm{S}_{7}$.

Now set $\bar{F}_{0}=C_{\bar{F}}(\bar{X})$, so that $\bar{F} / \bar{F}_{0} \cong \widetilde{F} \cong Z_{3}$. We shall argue that $\bar{F}_{0}=$ 1. Indeed, choose $v$ in $U-\langle u\rangle$, so that $[F, U]=[F, v]=F$ and $\bar{X}\langle\bar{v}\rangle \cong$ $S_{7}$. If $\bar{v}$ centralizes $\bar{F}_{0}$, then the subset of $\bar{F}$ inverted by $\bar{v}$ has order 3, whence $\bar{F}=\bar{F}_{0} \times[\bar{F}, \bar{v}]$, forcing $\bar{F}_{0}=1$. Hence we can assume that $\bar{v}$ does not centralize $\bar{F}_{0}$. Setting $\bar{F}_{1}=\left[\bar{F}_{0}, \bar{v}\right]$, it follows as with $\bar{F}$ that $\bar{F}_{1}$ does not centralize some component $\bar{X}_{1}$ of $L(\bar{N})$. Clearly $\bar{X} \neq \bar{X}_{1}$. Now the same reasoning as with $\bar{X}$ yields that $\bar{X}_{1} \cong A_{7}$ and $\bar{X}_{1}\langle\bar{v}\rangle \cong S_{7}$. However, it is immediate in this case that $\langle\bar{v}, \bar{t}\rangle$ centralizes an elementary subgroup of $\overline{X X}_{1}$ of order 16 disjoint from $\langle\bar{v}, \bar{t}\rangle$, whence $m(G) \geqslant 6$, which is not the case. This contradiction proves that $\bar{F}_{0}=1$ and so we conclude that $F / F \cap O\left(C_{G}(t)\right)$ has order 3.

It therefore remains only to prove that $t=u$. Consider $\tilde{Y}$ once again. We have that $\widetilde{E}$ centralizes $\widetilde{F} \cong Z_{3}$ and $\widetilde{E} \widetilde{F} \subseteq \widetilde{X}$. Since $E=C_{B}(F)$ and $m(B)=3$, it follows therefore from (2.30) that for some $b$ in $B-E$, we have $\widetilde{E} \subseteq \Lambda\left(C_{\tilde{X}}(\tilde{b})\right) \cong A_{5}$. Hence if $X$ denotes the component of $N$ which maps on $\bar{X}$, we obtain that $X_{0}=\Lambda\left(C_{X}(b)\right)$ maps on $\Lambda\left(C_{\tilde{X}}(\tilde{b})\right)$ and that $U_{0}=A \cap$ $X_{0}$ maps on $\tilde{E}$. Note also that as $C_{\tilde{X}}(\tilde{b}) \cong S_{5} \cong P G L(2,5), C_{X}(b)$ contains an involution $v$ such that $\tilde{X}_{0}\langle\bar{v}\rangle \cong \stackrel{X}{P} G L(2,5)$.

Now set $H=C_{G}(b)$ and $\bar{H}=H / O(H)$. We have that $\left\langle A, v, X_{0}, J\right\rangle \subseteq H$. We argue next that $\bar{X}_{0}$ and $\bar{J}$ lie in distinct components of $L(\bar{H})$. In any event, by $L$-balance, we have $\left\langle\bar{X}_{0}, \bar{J}\right\rangle \subseteq L(\bar{H})$. In addition, $m(G)=m(H)=5$. Since $J$ centralizes $B \cong E_{8}$, we easily conclude from this that the normal closure $\bar{L}_{1}$ of $\bar{J}$ in $L(\bar{H})$ consists of a single component (otherwise $m(H) \geqslant 6$ ). Likewise $L(\bar{H})$ possesses at most 2 components (using the fact that no component of $L(\bar{H})$ is isomorphic to $S L(2, q), q$ odd). Since $t$ normalizes $J, \bar{t}$ must leave $\bar{L}_{1}$ invariant and so $\bar{t}$ leaves each component of $L(\bar{H})$ invariant. We conclude therefore from (2.43) that also $\bar{X}_{0}$ lies in a unique component $\bar{L}_{2}$ of $L(\bar{H})$.

We must show that $\bar{L}_{1} \neq \bar{L}_{2}$, so assume the contrary. Since $X_{0}\langle v\rangle / O\left(X_{0}\right)$ $\cong P G L(2,5), \bar{L}_{1}$ cannot be of Ree type or isomorphic to $J_{1}$. Since $\bar{X}_{0}=$ $L\left(C_{\bar{L}_{1}}(\bar{t})\right)$, the only possibility is that $\bar{L}_{1} \cong L_{2}(5), L_{2}(25)$, or $A_{7}$. But then ${ }^{L_{1}} m\left(\bar{L}_{1}\right)=2$, whence $m\left(\bar{U}_{0} \bar{U}\right)=2$ and consequently $\underset{\widetilde{U}}{U}=U_{0}$. But now returning to $\widetilde{Y}$, it follows that $\widetilde{U}=\widetilde{U}_{0}=\widetilde{E}$ centralizes $\widetilde{F}$. However, this is 
a contradiction as $u$ is the only involution of $U$ which centralizes $\widetilde{F}$. This establishes our assertion.

Finally as $\bar{U} \subseteq \bar{J} \subseteq \bar{L}_{1}$ and $\bar{L}_{1}$ centralizes $\bar{L}_{2} \supseteq \bar{X}_{0}$, we have that $\bar{u}$ centralizes $\bar{X}_{0}$ and so $\bar{u}$ centralizes $X_{0} / O\left(X_{0}\right)$. But $t=u y$ for some $y$ in $E$ as $t \in T-E$. Considering $\widetilde{Y}$ again, our conditions imply that $\tilde{u}$ as well as $\tilde{t}$ centralizes $\widetilde{X}_{0}$. Thus also $\tilde{y}$ centralizes $\widetilde{X}_{0}$ and so $\widetilde{X}_{0} \subseteq L\left(C_{\tilde{X}}(\tilde{y})\right)$. However, we have shown above that $L\left(C_{\tilde{X}}(\tilde{y})\right)=1$ for $\tilde{y}$ in $\tilde{E}^{\#}$. Hence $\tilde{y}=1$. Since $E \cong \widetilde{E}$, this forces $y=1$ and we conclude that $t=u$, as required.

As a corollary, we have

LEMMA 6.6. Either $W_{A} \neq 1$ or $|F|=3$ and $F \nsubseteq O\left(C_{G}(u)\right)$.

Proof. Set $F_{0}=F \cap O\left(C_{G}(u)\right)$. By the preceding lemma, $F \subseteq O\left(C_{G}(t)\right)$ for $t$ in $T-\langle u\rangle$ and so $F_{0} \subseteq O\left(C_{G}(t)\right)$ for all $t$ in $T^{\#}$, whence $F_{0} \subseteq$ $\Delta_{G}(T)$. Thus $W_{A} \neq 1$ if $F_{0} \neq 1$. Since $F \neq 1$, it follows, in the contrary case, from the preceding lemma that $|F|=3$. By definition of $F_{0}$, we also then have $F \nsubseteq O\left(C_{G}(u)\right)$ and the lemma follows.

It thus remains to treat the latter case. We need some information about $C_{G}(u)$. Recall the groups $X$ and $Y$ of Lemma 6.5.

LEMMA 6.7. The following conditions hold:

(i) $X$ is a component of. $L_{u}$ and $\bar{X}=X / O(X) \cong A_{7}$;

(ii) An involution $v \in U-\langle u\rangle$ induces an outer automorphism on $\bar{X}$;

(iii) $E\langle v\rangle$ acts faithfully on $\bar{X}$;

(iv) $X=L_{u}$.

Proof. The first three assertions are contained in the proof of Lemma 6.5. Suppose $L_{u}$ has another component, $K$. If $Z(K / O(K)) \neq 1$, then by Proposition $4.1, K / O(K)$ is a covering group of $L_{3}(4)$ whence by $\left.(2.16), m\left(C_{G}(u)\right) \geqslant m(K)\right)$ $+m(X) \geqslant 7$ contradicting $m(A)=5$. Thus $Z(K / O(K))=1$ and $m(K) \geqslant 2$. Considering the action of $v$ on $X$, we see $U \cap X=U \cap K=1$ and $m\left(C_{G}(u)\right.$ ) $\geqslant m(U)+m(K)+m(X) \geqslant 6>m(\mathrm{H})$. Thus we must have $L_{\boldsymbol{u}}=X$.

Lemma 6.8. Either $W_{\dot{A}} \neq 1$, or $C_{D}(U)=C_{D}(J)$.

Proof. Assume $W_{A}=1$. By Lemma 6.4, $E \subset B$, and so by our choice of $E,\left[C_{D}(B), J\right]=1$. Thus $C_{D}(B) \subseteq C_{D}(J)$, and it suffices to show $C_{D}(U) \subseteq$ $C_{D}(B)$. If we let $F^{*}=\left[C_{D}(U), B\right]$, then by our initial assumption we need only show $F^{*} \subseteq \Delta_{T}$. If this inclusion fails, then just as in the proof of Lemma $6.5, F^{*}$ acts nontrivially on $\bar{X}^{*}=X^{*} / O\left(X^{*}\right)$ for some component $X^{*}$ of $C_{G}\left(t^{*}\right)$ with $t^{*} \in T-E$. Likewise $F^{*}=\left[F^{*}, B\right]$ implies $X^{*}$ is of type 
$A_{7}$, and for some $b^{*} \in B-E, X_{0}^{*}=\Lambda\left(C_{X^{*}}\left(b^{*}\right)\right)$ is of type $A_{5}$ and $E$ acts as inner automorphisms on $\bar{X}_{0}^{*}$. We will derive a contradiction.

First we claim $t^{*} \neq u$. If $t^{*}=u$, then by Lemma $6.7, X=X^{*}$ and $E^{*}=$ $E\langle v\rangle$ acts faithfully on $\bar{X}^{*}=X^{*} / O\left(X^{*}\right)$. It is easy to see that the image of $E^{*}$ is a self-centralizing subgroup of $\operatorname{Aut}\left(\bar{X}^{*}\right)$ contradicting the fact that $F^{*}$ and $E^{*}$ commute. Thus $t^{*} \neq u$, and as $t^{*} \in T-E$, Lemma 6.7 implies that $t^{*}$ acts as an inner automorphism on $\bar{X}$ and that $L_{2^{\prime}}\left(C_{G}\left(t^{*}\right) \cap L_{u}\right)=1$.

We shall obtain a contradiction by showing $L_{2^{\prime}}\left(C_{G}(u) \cap L_{t^{*}}\right) \neq 1$ whence $L_{2}\left(C_{G}\left(t_{*}\right) \cap L_{u}\right) \neq 1$ by $L$-balance. Let $H^{*}=C_{G}\left(b^{*}\right)$. Just as in the proof of Lemma $6.5, J$ lies in a component $L_{1}^{*}$ of $H^{*}$ and $X_{0}^{*}$ lies in a component $L_{2}^{*}$ of $H^{*}$. If $L_{1}^{*}=L_{2}^{*}$, then as $E$ acts faithfully on $\bar{X}_{0}^{*}, E$ acts faithfully on $L_{1}^{*}$. By (2.43) $X_{0}^{*}=L_{2^{\prime}}\left(C_{L_{1}^{*}}\left(t^{*}\right)\right)$ whence by (2.26) $L_{1}^{*}$ is of type $A_{5}, A_{7}$. $J_{1}$, or $L_{2}(25)$. In any case $(2.26)$ implies $C_{L_{1}^{*}}(E)$ is solvable. But by definition of $E, J \subseteq C_{G}(E) \cap L_{1}^{*}$, whence $C_{L_{1}^{*}}(E)$ is not solvable. Thus $L_{1}^{*} \neq L_{2}^{*}$ and $U \subseteq J \subseteq L_{1}^{*}$ implies $U$ centralizes $X_{0}^{*} / O\left(X_{0}^{*}\right)$. Thus $C_{G}(u) \cap C_{G}\left(t^{*}\right)$ covers $X_{0}^{*} / O\left(X_{0}^{*}\right)$ whence $C_{G}(u) \cap C_{G}\left(t^{*}\right)$ covers the image $\bar{X}_{0}^{*}$, of $X_{0}^{*}$ in $\bar{X}^{*}$. As $X$ is $A$-invariant by (2.37), (2.26) implies $\bar{X}_{0}^{*} \subseteq L\left(C_{\bar{X}^{*}}(u)\right)$, and we see that $L_{2}\left(C_{G}(u) \cap L_{t^{*}}\right) \neq 1$.

\section{Lemma 6.9. We have $W_{A} \neq 1$.}

Proof. By the preceding analysis, it remains to treat the case in which $\left[C_{D}(u), U\right]$ has order 3 and $C_{D}(U)=C_{D}(J)$. We shall argue that this case cannot arise and this will establish the lemma.

As $D=[D, J]$, we can find a factor group, $\bar{D}$, of $D$ such that $\bar{D}$ is an irreducible nontrivial $J$-module. As $\bar{D}=\left\langle C_{\bar{D}}(y) \mid y \in U^{*}\right\rangle,\left[C_{\bar{D}}(y), U\right] \neq 1$ for some $y \in U \#$ else $[\bar{D}, U]=1$. Since $\left|\left[C_{D}(u), U\right]\right|=3$ and the involutions of $U$ are conjugate in $J$, we conclude $\left|\left[C_{\bar{D}}(y), U\right]\right|=3$ for all $y \in$ $U^{\#}$. By Lemma 6.8, $\overline{C_{D}(U)} \subseteq \overline{C_{D}(J)} \subseteq C_{\bar{D}}(J)=1$, whence $C_{\bar{D}}(U)=1$. We conclude therefore from the well-known Brauer-Wielandt formula for the action of a four group on a group of odd order that $|\bar{D}|=3^{3}$. Thus $\bar{J}=J / C_{J}(\bar{D})$ is isomorphic to a nonsolvable perfect subgroup of $G L(3,3)$. However, as $\left.L_{3}(3) \cong S L(3,3)\right)$ is a minimal simple group, the only possibility is that $\bar{J} \cong L_{3}(3)$. But this is a contradiction as $L_{3}(3)$ has quasi-dihedral Sylow 2-subgroups of order 16 and class 3.

Since $W_{A}$ is a nontrivial subgroup of $G$ of odd order, $N_{G}\left(W_{A}\right)$ is a proper subgroup of $G$. Hence to complete the proof of Proposition 6.1 in the core case, it would suffice to prove that $V_{A}^{*} \subseteq N_{G}\left(W_{A}\right)$. Unfortunately the argu- 
ment to establish this again breaks down in certain situations when $m(A)=5$. To take these exceptions into account, we carry out the proof in two stages.

First of all, for $x$ in $A^{\#}$, we define $\widetilde{\Lambda}_{x}^{*}$ as follows: If $m(A)>5$, set $\tilde{\Lambda}_{x}^{*}=\Lambda_{x}^{*}$; while if $m(A)=5$, write $\Lambda_{x}^{*}=\Lambda_{x} D_{x}$, where $D_{x}$ is $A$-invariant of odd order and contains $O\left(C_{G}(x)\right.$ ) (which we can do by (2.50)) and set $\tilde{\Lambda}_{x}^{*}=$ $\widetilde{\Lambda}_{x} D_{x}$, where $\tilde{\Lambda}_{x}$ denotes the product of those components $K$ of $\Lambda_{x}$ such that either (a) $K$ is not of type $L_{2}(5)$ or $J_{1}$, (b) $K$ is of type $L_{2}(5)$ and $A \cap$ $K \notin M(K)$, or (c) $K$ is of type $L_{2}(5), A \cap K \in M(K)$, and there exists an involution $u$ in $C_{G}(x)$ which normalizes $K$ and $A$ such that $m\left(C_{A}(u)\right)=4$ and $K\langle u\rangle / O(K) \cong P G L(2,5)$.

Note that $D_{x}$ leaves each component of $\Lambda_{x}$ invariant. Moreover, if $L$ is a component of $\Lambda_{x}$ not in $\tilde{\Lambda}_{x}$, then $L$ is of type $L_{2}(5)$ or $J_{1}$ and $A \cap$ $L \in M(L)$. Since $L_{2}(5)$ and $J_{1}$ have no nontrivial outer automorphisms of odd order and have trivial 2-signalizers, it is immediate that $D_{x}$ centralizes $L / O(L)$. Hence $L$ normalizes $D_{x}$. Since $L$ also normalizes $\tilde{\Lambda}_{x}, \tilde{\Lambda}_{x}^{*} / \tilde{\Lambda}_{x}=$ $O\left(\Lambda_{x}^{*} / \tilde{\Lambda}_{x}\right)$, and we conclude that $\tilde{\Lambda}_{x}^{*}$ is normal in $\Lambda_{x}^{*}$, a key fact which we shall need for our final argument.

Finally if $B$ is any subgroup of $A$, we set

$$
\widetilde{V}_{B}^{*}=\left\langle\widetilde{V}_{b}^{*} \mid b \in B^{\#}\right\rangle
$$

In particular, if $m(A)>5, \widetilde{V}_{B}^{*}=V_{B}^{*}$ and $\widetilde{V}_{A}^{*}=V_{A}^{*}$.

We first prove

Lemma 6.10. We have $\widetilde{V}_{A}^{*} \subseteq N_{G}\left(W_{A}\right)$.

Proof. Set $N=N_{G}\left(W_{A}\right)$. By (2.57), $W_{A}=W_{B}$ for $B$ in $E_{4}(A)$. Since $N_{G}(B)$ normalizes $W_{B}$, it follows that $N_{G}(B) \subseteq N$ for all $B$ in $E_{4}(A)$. This conclusion will be critical for the proof.

For $x$ in $A^{\#}$, we must show that $\widetilde{\Lambda}_{x}^{*} \subseteq N$. We write $\widetilde{\Lambda}_{x}^{*}=\widetilde{\Lambda}_{x} D_{x}$, as above. Since $m(A) \geqslant 5$ and $D_{x}$ is $A$-invariant, we have $D_{x}=\left\langle C_{D_{x}}(B)\right| B \in$ $\left.E_{4}(A)\right\rangle$ and so $D_{x} \subseteq N$. Since $O\left(\widetilde{\Lambda}_{x}\right) \subseteq O\left(C_{G}(x)\right) \subseteq D_{x}$, it will therefore suffice to prove that $N$ covers $\tilde{\Lambda}_{x} / O\left(\tilde{\Lambda}_{x}\right)$.

Set $H=C_{G}(x), \bar{H}=H / O(H)$, and let $K$ be a component of $\tilde{\Lambda}(H)$. We set $A_{0}=\mathrm{C}_{A}(\bar{K})$. If $m\left(A_{0}\right) \geqslant 4$, then an element of $E_{4}(A)$ centralizes $\bar{K}$, so clearly $N$ covers $\bar{K}$. Therefore we can suppose that $m\left(A_{0}\right) \leqslant 3$. By (2.23), $m\left(A / A_{0}\right)=2$ or 3 . Since $m(A) \geqslant 5$, we have, in fact, $m\left(A_{0}\right)=2$ or 3 . We let $R$ be an $A$-invariant Sylow 2-subgroup of $K$.

Consider first the case that $m\left(A_{0}\right)=3$. Since $A \in M(G)$ and $R$ is $A$-invariant, $Z(R)$ contains an element $z$ of $A^{\#}$. Setting $B=A_{0}\langle z\rangle$, we have 
then that $m(B)=4$. But now if $y \in I(R), y$ centralizes $B$ and $m\left(A_{0}\langle y\rangle\right)=4$, so $W_{A_{0}(y)}=W_{B}=W_{A}$ by (2.57). In particular, it follows that $C_{G}\left(A_{0}\langle y\rangle\right) \subseteq$ $N$. However, $C_{\bar{K}}\left(A_{0}\langle\bar{y}\rangle\right)=C_{\bar{K}}(\bar{y})$ and so $N$ covers $C_{\bar{K}}(\bar{y})$ for every $u$ in $I(\bar{R})$. We conclude therefore from (2.35) that either $N$ covers $K$ or else $\bar{K} \cong$ $L_{2}(5)$.

Consider the latter case. Then $m(A)=5$. The same reasoning shows that $N$ covers $N_{\bar{K}}(\bar{R})$. If $A \cap K \notin M(K)$, then for some $a$ in $A^{\#}, \bar{K}\langle\bar{a}\rangle \cong$ $\operatorname{PGL}(2,5)$. Since $m\left(A_{0}\langle a\rangle\right)=4$, it follows as before that $N$ covers $C_{\bar{K}}(\bar{a})$. But $K=\left\langle N_{\bar{K}}(\bar{R}), C_{\bar{K}}(\bar{a})\right\rangle$ by $(2.35)$ and again we conclude that $N$ covers $\bar{K}$. Suppose finally that ${ }_{A} \cap K \in M(K)$. By assumption, $K$ is a component of $\tilde{\Lambda}_{x}$ and as $m(A)=5, H$ must contain an involution $u$ such that $m\left(C_{A}(u)\right)=4$ and $\bar{K}\langle\bar{u}\rangle \cong P G L(2,5)$. Since $\bar{K}\langle\bar{u}\rangle$ has dihedral Sylow 2 -subgroups of order $8, u$ does not centralize $A \cap K$, so $u$ must centralize $A_{0}$. Again it follows that $N$ covers $C_{\bar{K}}(\bar{u})$ and we conclude from (2.35) as in the preceding case that $N$ covers $\bar{K}$. Thus $N$ covers $\bar{K}$ in all cases when $m\left(A_{0}\right)=3$.

Suppose then that $m\left(A_{0}\right)=2$, whence $m(A)=5$ and $m\left(A / A_{0}\right)=3$. By (2.23), $m(A \cap R)=m(R)=m(K)$. By assumption, $\bar{K} \nRightarrow J_{1}$. If $\bar{K}$ is of Ree type, $\bar{K}=\left\langle O\left(C_{K}(U)\right) \mid U \in E_{2}(A \cap R)\right\rangle$ by (2.35). Since each $m\left(A_{0} U\right)=4$, we conclude at once that $N$ covers $\bar{K}$. Likewise if $\bar{K} \cong L_{2}\left(q^{2}\right), q$ odd, $q>3$, then (2.35) implies that $\bar{K}=\left\langle O\left(C_{\bar{K}}(a)\right) \mid a \in(A \cap R)^{\#}\right\rangle$. But each $O\left(C_{\bar{K}}(a)\right)$ is $A$-invariant and so is generated by its intersections with $C_{K}(B)$ for $B \in E_{4}(A)$. Again $N$ covers $\bar{K}$. Furthermore, if $\bar{K} \cong A_{7},(2.35)$ implies that $\bar{K}=$ $\left\langle N_{\bar{K}}(U) \mid U \in E_{2}(R)\right\rangle$. But as $R A / A_{0} \cong D_{8} \times Z_{2}$ in this case, we see that $R$ centralizes a four subgroup of $A / A_{0}$ and consequently $A_{1}=C_{A}(R) \cong E_{16}$. Since $A_{0} U$ centralizes $A_{1}$, we have, as usual, $W_{A_{0} U}=W_{A_{1}}=W_{A}$ and so each $N_{\bar{K}}(U)=N_{\bar{K}}\left(A_{0} U\right)$ is covered by $N$. Again we conclude that $N$ covers $\bar{K}$. However, as $m\left(A / A_{0}\right)=3$, it follows from (2.18) that these are the only possibilities for $\bar{K}$. Thus $N$ covers $K$ in all cases. Since $K$ was an arbitrary component of $\tilde{\Lambda}_{x}, N$ therefore covers $\tilde{\Lambda}_{x} / O\left(\tilde{\Lambda}_{x}\right)$ and the lemma is proved.

By the lemma, $\widetilde{V}_{A}^{*}$ is a proper subgroup of $G$ and hence so is $N_{G}\left(V_{A}^{*}\right)$. Thus the following lemma will complete the proof of Proposition 6.1 in the core case.

Lemma 6.11. We have $V_{A}^{*} \subseteq N_{G}\left(\tilde{V}_{A}^{*}\right)$.

Proof. If $m(A)>5$, then $\widetilde{V}_{A}^{*}=V_{A}^{*}$, so the lemma is obvious. Hence we can assume that $m(A)=5$.

The proof will depend upon what we may call $\tilde{\Lambda}^{*}$-balance and $\tilde{\Lambda}^{*}$-generation: Namely, if $x, y \in \mathrm{A}^{\#}$ and $B \in E_{3}(A)$, then we have 


$$
\Lambda^{*}\left(\tilde{\Lambda}_{x}^{*} \cap C_{G}(y)\right) \subseteq \tilde{\Lambda}_{y}^{*} \text { and } \tilde{\Lambda}_{x}^{*}=\left\langle\Lambda^{*}\left(\tilde{\Lambda}_{x}^{*} \cap C_{G}(b)\right) \mid b \in B^{\#}\right\rangle
$$

The equality is a direct consequence of (2.54) and the definition of $\widetilde{\Lambda}_{x}^{*}$. Likewise the inclusion is easily reduced using (2.50(iii)) and (2.51) (cf. the proof of (2.53)) to the following assertion:

$$
\Lambda\left(\tilde{\Lambda}_{x} \cap C_{G}(y)\right) \subseteq \tilde{\Lambda}_{y},
$$

which we proceed to establish.

Let $K$ be a component of $\tilde{\Lambda}_{x}$. Then $y$ leaves $K$ invariant. Set $J=$ $\Lambda\left(C_{K}(y)\right)$. We know from $\Lambda$-balance that either $J=1$ or $J$ lies in a unique component $L$ of $\Lambda_{y}$. To establish our assertion, we need only show when $J \neq$ 1 that $L \subseteq \widetilde{\Lambda}_{y}$. Suppose false for some $K, J$ and $L$, in which case $L$ is of type $J_{1}$ or $L_{2}(5)$. Consider the first possibility. Since $K \subseteq \widetilde{\Lambda}_{x}, K$ is not of type $J_{1}$ and hence neither is $J$, so $J$ is of type $L_{2}(5)$ (as $J=L_{2}\left(C_{L}(x)\right)$ ). Hence $K$ is of type $L_{2}(5), L_{2}(25)$, or $A_{7}$. However, in the last two cases $y$ centralizes an involution $u$ of $K$ such that $J\langle u\rangle / O(J) \cong P G L(2,5)$. But then $u$ acts on $L$ and induces an outer automorphism of $L / O(L)$ of order 2, contrary to the fact that $J_{1}$ possesses no such automorphism. Hence $K$ is of type $L_{2}(5)$, whence $J$ covers $K / O(K)$. By (2.37), $A \cap L \in M(L)$ and this implies that $A \cap J \in M(J)$, whence $A \cap K \in M(K)$. However, as $K \subseteq \tilde{\Lambda}_{x}$ and $m(A)$ $=5$, it follows that $C_{G}(x)$ contains an involution $u$ such that $m\left(C_{A}(u)\right)=4$ and $K\langle u\rangle / O(K) \cong P G L(2,5)$. But if we set $A_{0}=C_{A}(K / O(K))$, our conditions force $y$ to lie in $A_{0}$ and $u$ to centralize $A_{0}$. Again $u$ acts on $L$ and we reach the same contradiction.

Therefore $L$ is of type $L_{2}(5)$, whence $J$ covers $L / O(L), J$ is of type $L_{2}(5)$, and $K$ is of type $A_{7}, L_{2}(25)$, or $L_{2}(5)$. In the first two cases, with $A_{0}$ as above, we have that $B=A_{0}\langle y\rangle \cong E_{8}$. As $L \nsubseteq \widetilde{\Lambda}_{y}, A \cap L \in M(L)$, whence $A \cap J \in M(J)$ and by (2.26), $K$ contains an involution $u$ which centralizes $B$, normalizes $A \cap J$, and is such that $J\langle u\rangle / O(K) \cong P G L(2,5)$. We see then that $m\left(C_{A}(u)\right)=4$ and that $u$ acts on $L$ with $L\langle u\rangle / O(L) \cong P G L(2,5)$. Since $A \cap L \in M(L)$, we conclude that $L \subseteq \widetilde{\Lambda}_{y}$, contrary to our assumption. Thus $K$ is of type $L_{2}(5)$, whence $J$ covers $K / O(K)$. But then if $A \cap K \notin M(K)$, it follows that also $A \cap L \notin M(L)$, and again $L \subseteq \widetilde{\Lambda}_{y}$, a contradiction. Hence $A \cap$ $K \in M(K)$. Since $K \subseteq \widetilde{\Lambda}_{x}, C_{G}(x)$ contains an involution $u$ with the usual properties. Again it follows that $u$ centralizes $y$, so that $u$ acts on $L$ and $L\langle u\rangle / O(L) \cong P G L(2,5)$. Since $m\left(C_{A}(u)\right)=4$ and $A \cap L \in M(L)$, we reach the same contradiction in this case as well. Thus the desired relations are established.

For any $x$ in $A^{\#}$ and any $B$ in $E_{3}(A)$, it is immediate from these relations that $\widetilde{\Lambda}_{x}^{*} \subseteq \widetilde{V}_{B}^{*}$. Hence $\widetilde{V}_{A}^{*} \subseteq \widetilde{V}_{B}^{*}$ and as the reverse inclusion is obvious, 
we therefore conclude that

$$
\tilde{V}_{B}^{*}=\tilde{V}_{A}^{*} \text { for any } B \in E_{3}(A) .
$$

Now we can quickly establish the lemma. Let $x \in A^{\#}$. We must show that $\Lambda_{x}^{*}$ normalizes $\widetilde{V}_{A}^{*}$. We have that $\widetilde{\Lambda}_{x}^{*} \subseteq \widetilde{V}_{A}^{*}$. Hence by the definition of $\widetilde{\Lambda}_{x}^{*}$, it will suffice to prove that any component $K$ of $\Lambda_{x}$ of type $L_{2}(5)$ or $J_{1}$ normalizes $\widetilde{V}_{A}^{*}$. In the first case, set $B=C_{A}(K / O(K))$, so that $m(B)=3$. Then $J=L\left(C_{K}(B)\right)$ covers $K / O(K)$. In the second case, set $A_{0}=C_{A}(K / O(K))$, so that $m\left(A_{0}\right)=2$. By $(2.35)$, we have that $\left\langle L\left(C_{K}(B)\right) \mid B \in E_{3}(A), B \supset A_{0}\right\rangle$ covers $K / O(K)$ with each $J=L\left(C_{K}(B)\right)$ of type $L_{2}(5)$. Since $O(K) \subseteq \widetilde{V}_{A}^{*}$, it follows therefore in either case that we need only show for each such $B$ and corresponding $J$ that $J$ normalizes $\widetilde{V}_{A}^{*}$.

But for $b$ in $B^{\#}, \Lambda$-balance implies that $J \subseteq \Lambda_{b} \subseteq \Lambda_{b}^{*}$. We have shown earlier that $\widetilde{\Lambda}_{b}^{*} \triangleleft \Lambda_{b}^{*}$, so $J$ normalizes each $\widetilde{\Lambda}_{b}^{*}$. Hence $J$ normalizes $\widetilde{V}_{B}^{*}$. However, $\widetilde{V}_{B}^{*}=\widetilde{V}_{A}^{*}$ and so $J$ normalizes $\widetilde{V}_{A}^{*}$, as required. This completes the proof of the lemma and with it the proof of Proposition 6.1 in the core case.

We turn now to the semisimple case, which is considerably easier than the core case. We begin, however, with a preliminary definition.

Let $K$ be a component of $\Lambda_{a}$ for $a$ in $A^{\#}$. Then $K$ is quasisimple and $K / O(K) \in A_{1}$. (Actually we have $O(K)=1$ unless $K$ is of type $A_{7}$, as the other elements of $A_{1}$ possess no nontrivial perfect central extensions of odd order.) In any event, it follows that $C_{A}(K)=C_{A}(K / O(K))$ as $O(K) \subseteq$ $Z(K)$. Now for any $y$ in $C_{A}(K)^{\#}$, we know by $\Lambda$-balance that $K$ lies in a unique component $L$ of $\Lambda_{y}$. If $K=L$ for each such $y$, we shall say that $K$ is a nonembedded component of $\Lambda_{a}$. In particular, if we choose $a$ and $K$ so that $K / O(K)$ is an element of $L_{1}(G ; A)$ of maximal order, then certainly $K$ will be nonembedded. Moreover, it is easy to see that for any $a$ in $A^{\#}$ and any component $K$ of $\Lambda_{a}, K$ is contained in some nonembedded component.

Among all nonembedded components we now choose $a$ and $K$ so that $m\left(C_{A}(K)\right)$ is maximal. Since $a$ centralizes $K$ and $G$ is simple, both $K$ and $N_{G}(K)$ are proper subgroups of $G$. Hence the following result will establish Proposition 6.1 in the present case:

Lemma 6.12. We have $V_{A}^{*} \subseteq N_{G}(K)$.

Proof. Set $N=N_{G}(K)$ and $B=C_{A}(K)$. As usual, $m(B) \geqslant 2$ and $m(A / B)=2$ or 3. Since $K$ is nonembedded, it follows that for $b$ in $B^{\#}, K$ is a component of $\Lambda_{b}$.

Let $D$ be any $A$-invariant subgroup of $G$ of odd order. We argue that $D \subseteq N$. Since $D=\left\langle C_{D}(b) \mid b \in B^{\#}\right\rangle$, it suffices to treat the case in which $D$ centralizes some $b$ in $B^{\#}$. Then $D \subseteq C_{G}(b)$ and so $D$ induces a permutation 
of the components of $\Lambda_{b}$, one of which is $K$. We know that $K$ contains an element $x$ of $A^{\#}$ and that $|Z(K)|$ is odd. Hence either $D$ normalizes $K$ or $[d, x]=x^{d} x$ is of even order for some $d$ in $D^{\#}$. However, $|[D, A]|$ is odd as $D$ is $A$-invariant. Thus $D \subseteq N$, as asserted.

To prove the lemma, we must show that $\Lambda_{x}^{*} \subseteq N$ for $x$ in $A^{\#}$. We have that $\Lambda_{x}^{*}=\Lambda_{x} D_{x}$, where $D_{x}$ is $A$-invariant of odd order by (2.50). Hence by the preceding paragraph, we need only show that $\Lambda_{x} \subseteq N$. Consider first the case that $m(B) \geqslant 3$. If $J$ is a component of $\Lambda_{x}$, then $B$ acts on $J$ and so

$$
J=\left\langle\Lambda\left(C_{J}(b)\right) O\left(C_{J}(b)\right) \mid b \in B^{\#}\right\rangle
$$

by (2.34) and (2.27(i)). But each $O\left(C_{J}(b)\right)$, being $A$-invariant of odd order, lies in $N$. Moreover, by $\Lambda$-balance, $\Lambda\left(C_{J}(b)\right) \subseteq \Lambda_{b}$. However, $\Lambda_{b} \subseteq N$ as $K$ is a component of $\Lambda_{b}$, so each $\Lambda\left(C_{J}(b)\right) \subseteq N$. Thus $J \subseteq N$ and we conclude that $\Lambda_{x} \subseteq N$.

Suppose then that $m(B)=2$, whence $m(A)=5$. Thus again the rank 5 case is exceptional. Clearly if $N$ contains every nonembedded component, it will contain $\Lambda_{x}$ for each $x$ in $A^{\#}$. Hence for $x$ in $A^{\#}$, we need only prove that any nonembedded component $J$ of $\Lambda_{x}$ lies in $N$. Suppose $J \cong L_{2}(q)$, $q$ odd, $q>9, J_{1}$, or is of Ree type. Then by (2.35), we again obtain (*) and it follows as in the preceding case that $J \subseteq N$. On the other hand, if $J \cong L_{2}(5)$ or $L_{2}(7)$, then $m\left(C_{A}(J)\right)=3$ and our choice of $K$ would be contradicted. Hence these two cases cannot arise. There thus remains only the single possibility that $J / O(J) \cong A_{7}$, and that $m\left(C_{A}(J)\right)=2$. Since $J$ satisfies the same conditions as $K$, we could have begun with $J$ instead of $K$, if we had wished, and so without loss we can also assume that $K / O(K) \cong A_{7}$.

We set $E=C_{A}(J)$. If some $b$ in $B^{\#}$ lies in $E$, then $J$ lies in $\Lambda_{b}$ and so $J$ normalizes $K$. Hence we can also assume that $B \cap E=1$. Hence if we set $\overline{J A}=J A / O(J) E$, we have that $\overline{J A} \cong S_{7}$. It follows therefore from (2.22) that $N_{\bar{J}}(\bar{A})$ is generated by its involutions. Let then $u$ be an arbitrary involution of $N_{J}(A)$. Since $u$ centralizes $E$ and a Sylow 2-subgroup of $\overline{J A}$ is isomorphic to $D_{8} \times E_{2}$, it follows that $\left|A: C_{A}(u)\right| \leqslant 2$. Since $m(B)=2$, this implies that $C_{B}(u) \neq 1$. Choosing $b$ in $C_{B}(u)^{\#}$, we have then that $K$ is a component of $L_{b}$ and $u \in C_{G}(b)$. Since $K$ has nonabelian Sylow 2-subgroups, $u$ must normalize $K$ by (2.13), whence $u \in N$. Thus $\left\langle u \mid u \in I\left(N_{J}(A)\right)\right\rangle \subseteq$ $N$. Since $N_{\bar{J}}(\bar{A})$ is generated by its involutions, we conclude that $N$ covers $N_{J}(A) O(J) / O(J) \cong N_{\bar{J}}(\bar{A})$.

Now choose $v$ to be an involution of $N_{J}(A)$ such that $(A \cap J)\langle v\rangle \cong$ $D_{8}$, which is possible as $A \cap J \cong E_{4}$ and a Sylow 2-subgroup of $J$ is isomor- 
phic to $D_{8}$. Then $\left|A: C_{A}(v)\right|=2$ as $v$ does not centralize $A \cap J$ and consequently $A^{*}=C_{A}(v)\langle v\rangle \cong E_{32}$, whence $A^{*} \in M(G)$. As above, $v$ centralizes some element $b$ of $B^{\#}$, so $b \in A^{*}$. Thus $K \in L\left(G ; A^{*}\right)$. Furthermore, as $K / O(K) \cong A_{7}, K$ is certainly a nonembedded element of $L\left(G ; A^{*}\right)$. Hence all the above considerations hold equally well for $A^{*}$ in place of $A$. Since $v \in$ $C_{G}(x)$, clearly $x \in A^{*}$ and we conclude therefore by the same reasoning that $N$ covers $N_{J}\left(A^{*}\right) O(J) / O(J) \cong N_{\bar{J}}\left(\bar{A}^{*}\right)$.

On the other hand, as $B \cap E=1$ and $m(B)=2$, some $b_{0}$ in $B^{\#}$ induces a nontrivial inner automorphism of $J$. We have already seen that every 2 element of $C_{G}\left(b_{0}\right)$ leaves $K$ invariant by (2.13). But $L_{b_{0}}$ has at most two components isomorphic to $K$ as $m(G)=5$, so every element of odd order in $C_{G}\left(b_{0}\right)$ also leaves $K$ invariant. Thus $K \triangleleft C_{G}\left(b_{0}\right)$ and consequently $N$ also covers $C_{\bar{J}}\left(\bar{b}_{0}\right)$. However, by $(2.35(\mathrm{ix}))$, we have

$$
\bar{J}=\left\langle N_{\bar{J}}(\bar{A}), N_{\bar{J}}\left(\bar{A}^{*}\right), C_{\bar{J}}\left(\bar{b}_{0}\right)\right\rangle .
$$

Our argument therefore yields that $N$ covers $\bar{J}$. Since $O(J) \subseteq Z(J)$ and $J$ is perfect, this forces $N$ to contain $J$ and the lemma is proved.

This finally completes the proof of Proposition 6.1.

7. The normalizer of the odd $A$-hull. With the aid of Proposition 6.1, we shall prove in this section

Proposition 7.1. Either $L_{1}(G)$ is empty or consists of an evenly embedded $L_{2}(5)$.

We proceed by contradiction in a long sequence of lemmas. We preserve the notation $V_{Q}^{*}$ for the odd $Q$-hull defined in the preceding section. We fix $A$ in $M(G)$ and let $S$ be a Sylow 2-subgroup of $G$ containing $A$. Then $A \triangleleft S$ and by Proposition 5.14, we have $m(S)=m(A) \geqslant 5$. Moreover, by Proposition 6.1, we know that the odd $A$-hull $V_{A}^{*}$ is a proper subgroup of $G$. We set

$$
M=N_{G}\left(V_{A}^{*}\right) \text {. }
$$

Our goal will be to prove that $M$ is strongly embedded in $G$. Bender's theorem will then yield an immediate contradiction. Note that $V_{A}^{*}$ is clearly invariant under $N_{G}(A)$. Since $A \triangleleft S$, it follows that $S \subseteq M$.

Our first lemma will imply that $M$ is a proper subgroup of $G$.

Lemma 7.2. For some $a$ in $A^{\#}$, we have $\Lambda_{a} \neq 1$.

Proof. Suppose false. Since Proposition 7.1 is assumed false, $\Lambda_{x} \neq 1$ for some $x$ in $I(S)$. Setting $B=C_{A}(x)$, we have that $m(B) \geqslant 3$ as $A \triangleleft S$ and 
$m(A) \geqslant 5$. Now $B$ acts on $\Lambda_{x}$ and so by (2.49), we have $K_{b}=\Lambda\left(C_{\Lambda_{x}}(b)\right) \neq$ 1 for some $b$ in $B^{\#}$.

Since $B \subseteq A$, we have $\Lambda_{b}=1$ by assumption. Hence no component of $K_{b}$ lies in $\Lambda_{b}$. In particular, $G$ is not $\Lambda$-balanced. It follows therefore from Lemma 5.1 that, in fact, $m(A) \geqslant 7$, whence $m(B) \geqslant 4$. But now (2.49) yields that either all components of $\Lambda_{x}$ are of type $L_{2}(5)$ or else for some $b$ in $B^{\#}, K_{b}$ has a component which is not of type $L_{2}(5)$. However, in the latter case, it would follow from (2.46) that $\Lambda_{b} \neq 1$. We thus conclude that each component of $\Lambda_{x}$ is, in fact, of type $L_{2}(5)$.

This analysis applies to each $x$ in $I(S)$ and so our argument yields that $A_{1}(G)$ consists only of $L_{2}(5)$. Again as Proposition 7.1 is assumed false, $L_{2}(5)$ is not evenly embedded in $G$ and it follows from the definition that for some $x$ in $I(S), H=C_{G}(x)$ is not 1-balanced as a $C_{2}$-group. Furthermore, replacing $x$ by a conjugate in $S$, we can also suppose that $P=C_{S}(x)$ is a Sylow 2-subgroup of $H$.

Set $\bar{H}=H / O(H)$. Since $H$ is not 1-balanced as a $C_{2}$-group, $\Lambda_{x}$ has a component $F$ such that $N_{\bar{H}}(\bar{F}) / C_{\bar{H}}(\bar{F}) \cong P G L(2,5)$. Hence $T=P \cap F$ is a four group and there exists $y \in P-T$ with $y$ normalizing, but not centralizing $T$. To complete the proof of the lemma, we shall now contradict this conclusion. Since $P \subseteq S$, it will obviously suffice to prove that $T \subseteq Z(S)$.

By (2.13), $F$ is invariant under a maximal subgroup $B_{0}$ of $B$. Since $m\left(\operatorname{Aut}\left(L_{2}(5)\right)\right)=2$ and $m\left(B_{0}\right) \geqslant 3$, we have $C_{B_{0}}(\bar{F}) \neq 1$. and consequently $F_{0}=\Lambda\left(C_{F}(b)\right) \neq 1$ for some $b$ in $B_{0}^{\#}$. Moreover, $T \subseteq F_{0}$. Since $\Lambda_{b}=1$, (2.42) and (2.46) now yield that $F_{0} \subseteq J$ for some component $J$ of $L_{b}$ of type $L_{2}(16)$ on which $x$ acts irregularly. On the other hand, $A \subseteq N=C_{G}(b)$ and as $A \in M(G)$ and $J$ has an elementary abelian Sylow 2-subgroup, we conclude from (2.37) that $A_{0}=A \cap J \in M(J)$. Then $x$ normalizes $A_{0}$ and $\left[A_{0}, x\right] \subseteq S^{\prime} \subseteq$ $Z(S)$. Hence we need only show that $T \subseteq\left[A_{0}, x\right]$.

Finally setting $\bar{N}=N / O(N)$, we have that $C_{\bar{J}}(\bar{x}) \cong L_{2}(5)$ and has $\left[\bar{A}_{0}, \bar{x}\right]$ as a Sylow 2-subgroup. But $T \subseteq F_{0} \subseteq J$ and so $T \subseteq S \cap J=A_{0}$. Since $\bar{F}_{0} \subseteq C_{\bar{J}}(\bar{x})$, it follows that $\bar{T} \subseteq\left[\bar{A}_{0}, \bar{x}\right]$. Whence $T \subseteq\left[A_{0}, x\right]$ and the lemma is proved.

As a corollary we have

LEMMA 7.3. $M$ is a proper subgroup of $G$.

Proof. By the preceding lemma and Proposition 6.1, $V_{A}^{*}$ is a nontrivial proper subgroup of $G$. Since $G$ is simple, this forces $M \subset G$.

The first major step in showing that $M$ is strongly embedded in $G$ is to 
prove that $\Gamma_{S, 3}(G) \subseteq M$. Before attacking this problem, we need a preliminary result.

LEMMA 7.4. If for some $x$ in $I(S), L_{x}$ has a component of type $L_{2}(16)$ on which an involution of $C_{G}(x)$ acts irregularly, then some involution of $Z(S)$ has the same property.

Proof. Without loss we can assume that $P=C_{S}(x)$ is a Sylow 2-subgroup of $H=C_{G}(x)$. Choose $x$ so that $|P|$ is maximal and suppose by way of contradiction that $P \subset S$. Let $K$ be a component of $H$ of type $L_{2}(16)$ on which an involution $y$ of $P$ acts irregularly. Set $T=P \cap K$, so that $T \in$ $S(K)$. Since $1 \neq[T, y] \subseteq S^{\prime} \subseteq Z(S), P$ leaves $[T, y]$ invariant and therefore $P$ leaves $K$ invariant. Hence if we set $R=C_{P}(K / O(K))$, we have that $R \triangleleft P$. In addition, (2.19) implies that $|P: T R|=2$, so that $P=T R\langle y\rangle$.

Let $z$ be an involution of $R \cap Z(P)$. We shall argue that $L_{z}$ satisfies the same conditions as $L_{x}$. We set $N=C_{G}(z), \bar{N}=N / O(N)$, and $K_{0}=L_{2^{\prime}}\left(C_{K}(z)\right)$, so that $K_{0}$ covers $K / O(K)$ and $K_{0}$ is $P$-invariant. Then $P \subseteq N$ and by (2.43) the normal closure $J$ of $K_{0}$ in $L_{z}$ either consists of a single component or of two components $J_{1}$ and $J_{2}$ interchanged by $x$. Moreover, it follows in either case from (2.43) that $\bar{K}_{0}=L\left(C_{\bar{J}}(\bar{x})\right)$, so that in the latter case $J_{1}$ and $J_{2}$ are each of type $L_{2}(16)$, while in the former case, $J$ is either of type $L_{2}(16)$ or $L_{2}\left(2^{8}\right)$ by (2.26). Furthermore, as $P$ leaves $K_{0}$ invariant and $P \subseteq N, P$ leaves $J$ invariant in either case.

If $J$ has two components, then $y$ or $y x$ leaves both $J_{1}$ and $J_{2}$ invariant. Correspondingly set $y_{1}=y$ or $y x$. Then $C_{\bar{K}_{0}}\left(\bar{y}_{1}\right) \cong L_{2}(5)$ and as $\bar{K}_{0}=L\left(C_{\bar{J}}(\bar{x})\right)$, it follows that $C_{\bar{J}}\left(\bar{y}_{1}\right) \cong L_{2}(5), i=1,2$. Thus the involution $y_{1}$ acts irregularly on $J_{1}$ and so $L_{z}$ has the required property in this case.

Suppose, on the other hand, that $J$ consists of a single component. If $J$ is of type $L_{2}(16)$, then $\bar{J}=\bar{K}_{0}$ so $y$ acts irregularly on $J$ and again we are done. Assume finally that $\bar{J}$ is of type $L_{2}\left(2^{8}\right)$, in which case $x$ induces a field automorphism of $\bar{J}$ of order 2. By (2.19) we have $N_{\bar{N}}(\bar{J})=\langle\bar{x}\rangle \bar{J} C_{\bar{N}}(\bar{J})$. Since $\bar{P}$ leaves $\bar{J}$ invariant, it follows that $\bar{y}_{1}=\bar{y}$ or $\overline{y x}_{\bar{N}}$ induces an inner automorphism on $\bar{J}$. But as $C_{\bar{J}}\left(\bar{y}_{1}\right) \supseteq C_{\bar{K}_{0}}\left(\bar{y}_{1}\right) \cong L_{2}(5)$, this is clearly possible only if $\bar{y}_{1}$ centralizes $\bar{J}$. But then $\bar{y}_{1}$ and hence also $\bar{y}$, centralizes $\bar{K}_{0}$, which is not the case. Thus this case cannot occur and so our assertion is proved.

Since $P \subseteq N=C_{G}(z)$, we conclude now from our maximal choice of $P$ that $P$ is a Sylow 2-subgroup of $N$. Since $P \subset S$, it follows, in particular, that $z \notin Z(S)$. Thus $R \cap Z(S)=1$. Since $\mho^{1}(R)$ and $[R, y]$ lie in $Z(S)$, we obtain that $R$ is elementary abelian and $y$ centralizes $R$. Since $P=R T\langle y\rangle$ 
and $R T=R \times T$, we conclude that $R \subseteq Z(P)$. Maximality of $P$ now yields that every involution of $R$ is extremal in $S$ and has $P$ as a Sylow 2-subgroup of its centralizer.

This in turn implies that $R \cap R^{w}=1$ for $w$ in $S-P$. Indeed, if not, there exists $u$ in $R^{\#}$ such that $u^{w} \in R$. But then $[u, w] \in R \cap S^{\prime} \subseteq R \cap$ $Z(S)=1$, so $w$ centralizes $u$, contrary to the fact that $P \in S\left(C_{G}(u)\right)$.

Setting $T_{0}=[T, y]$, we have that $T_{0} \cong E_{4}$ and that $T_{0}=T \cap Z(P)$. Thus $Z(P)=T_{0} \times R$. But $P \triangleleft S$ as $Z(S) \subseteq P$ and so if $w \in S-P$, then $R^{w} \subseteq Z(P)=T_{0} R$. Since $R \cap R^{w}=1$, we conclude now that $R \cong E_{2}$ or $E_{4}$. Since $T_{0} \subseteq Z(S) \subseteq Z(P)$, it also follows that $T_{0}=Z(S)$. But now we can derive a contradiction exactly as in the corresponding portion of Lemma 5.1, by showing, on the one hand, that $|S: P| \leqslant 4$; while, on the other, that $|S: P| \geqslant 16$.

In proving that $\Gamma_{S, 3}(G) \subseteq M$, we shall divide the analysis into two cases:

Regular case. For each $a$ in $A^{\#}$, no involution of $C_{G}(a)$ acts irregularly on a component of $L_{a}$.

Irregular case. For some $a$ in $A^{\#}$, an involution of $C_{G}(a)$ acts irregularly on a component of $L_{a}$.

We first consider the regular case. Since $Z(S) \subseteq A$, the preceding lemma yields that for every $x$ in $I(S)$, no involution of $C_{G}(x)$ acts irregularly on any component of $L_{x}$. Combining this with (2.53), we obtain

LEMMA 7.5. $G$ is $\Lambda^{*}$-balanced in the regular case.

We can now establish the desired assertion with a straightforward application of the signalizer functor method.

LEMMA 7.6. $\Gamma_{S, 3}(G) \subseteq M$ in the regular case.

Proof. We argue first that for all $X, Y$ in $E(S)$ with $m(X) \geqslant 3$ and $m(Y) \geqslant 3$, we have $V_{X}^{*}=V_{Y}^{*}$.

Indeed, as $m(S) \geqslant 5, S$ is 3-connected by (2.6) and consequently it will suffice to prove the given equality when $X$ and $Y$ centralize each other. By symmetry, we need only show that $V_{X}^{*} \subseteq V_{Y}^{*}$ and hence that

$$
\Lambda_{x}^{*} \subseteq V_{Y}^{*} \text { for each } x \text { in } X^{\#} \text {. }
$$

But $Y$ acts on $\Lambda_{x}^{*}$ as $Y$ centralizes $X$. Since $m(Y) \geqslant 3$, it follows now by $\Lambda^{*}$-generation $(2.54)$ that

$$
\Lambda_{x}^{*}=\left\langle\Lambda_{x}^{*} \cap C_{G}(y)\right)\left|y \in Y^{\#}\right\rangle .
$$

However, each term on the right lies in the corresponding $\Lambda_{y}^{*}$ by $\Lambda^{*}$-balance 
and so lies in $V_{Y}^{*}$, giving the desired inclusion $\Lambda_{x}^{*} \subseteq V_{Y}^{*}$.

Now consider $Q \subseteq S$ with $m(Q) \geqslant 3$ and let $B \in E_{3}(Q)$. Then for $u$ in $N_{G}(Q)$, we have that $B^{u} \subseteq Q$, so $B$ and $B^{u}$ lie in $E_{3}(S)$. Since also $A \in$ $M(S)$ with $m(A) \geqslant 3$, the equality we have just established yields

$$
V_{B}^{*}=V_{B}^{*}=V_{A}^{*} .
$$

However, it is immediate from the definition of $V_{B}^{*}$ that

$$
V_{B}^{*}=\left(V_{B}^{*}\right)^{u}
$$

Together these equalities imply that $u$ normalizes $V_{A}^{*}$. Thus $u \in M$ and so $N_{G}(Q) \subseteq M$. Since $Q$ was an arbitrary subgroup of $S$ with $m(Q) \geqslant 3$, we conclude now from the definition that $\Gamma_{S, 3}(G) \subseteq M$.

We shall establish the desired assertion by showing now that the irregular case, in fact, cannot occur. In Lemmas 7.7-7.15, we assume that we are in the irregular case.

First of all, $G$ is $\Lambda^{*}$-balanced with respect to $A$ by (2.53). Hence the argument of the preceding lemma yields

Lemma 7.7. If $B \subseteq A$ with $m(B) \geqslant 3$, then $V_{B}^{*}=V_{A}^{*}$ and $N_{G}(B) \subseteq M$.

We also have

LeMmA 7.8. We have $m(Z(S)) \geqslant 3$ and $m(S) \geqslant 7$.

Proof. By Lemma 7.4, there exists $z$ in $Z(S)^{\#}$ such that $L_{z}$ has a component $K$ of type $L_{2}(16)$ on which an involution $y$ of $C_{G}(z)$ acts irregularly. Since $S \subseteq C_{G}(z)$, we can take $y$ to lie in $S$. Then $T=S \cap K \cong$ $E_{16}$ and $T_{0}=[T, y] \subseteq Z(S)$ with $T_{0} \cong E_{4}$. Since $z \notin T_{0},\langle z\rangle T_{0}$ is thus a subgroup of $Z(S)$ of rank 3 and so $m(Z(S)) \geqslant 3$. Furthermore, the assertion $m(S) \geqslant 7$ follows from Lemma 5.1.

We set $Z=Z(S)$ and next prove

Lemma 7.9. We have $V_{Z}^{*}=V_{A}^{*}=\Lambda^{*}(M)$.

Proof. First, Lemmas 7.7 and 7.8 immediately give $V_{Z}^{*}=V_{A}^{*}$. Since $m(Z) \geqslant 3$, it follows from $\Lambda^{*}$-generation that $\Lambda^{*}(M)$ is generated by its subgroups $K_{z}^{*}=\Lambda^{*}\left(\Lambda^{*}(M) \cap C_{G}(z)\right)$ for $z$ in $Z^{\#}$. But $S \subseteq M$ and so each $K_{z}^{*}$ is $S$-invariant. Hence by $(2.50)$ applied to $K_{z}^{*} S$, we have that $K_{z}^{*}=K_{z} D_{z}$, where $K_{z}=\Lambda\left(K_{z}^{*}\right)$ and $D_{z}$ is $S$-invariant of odd order. Since $S \subseteq C_{G}(z)$, we conclude now 
from (2.48) that $K_{z} \subseteq \Lambda_{z}$ and from (2.51(i)) that $D \subseteq \Lambda_{z}^{*}$. Thus each $K_{z}^{*} \subseteq$ $\Lambda_{z}^{*} \subseteq V_{Z}^{*}=V_{A}^{*}$ and so $\Lambda^{*}(M) \subseteq V_{A}^{*}$.

For $a$ in $A^{\#}$, we have that $\Lambda_{a}^{*} \subseteq M$. If $a \in Z^{\#},(2.50)$ implies that $\Lambda_{a}^{*}=\Lambda_{a} D_{a}$, where $D_{a}$ is $A$-invariant of odd order. Another application of (2.48) yields that $\Lambda_{a} \subseteq \Lambda(M)$. Furthermore, it follows from (2.50(i)) that $D_{a}$ $\subseteq \Lambda^{*}(M)$. Hence each $\Lambda_{a}^{*} \subseteq \Lambda^{*}(M)$ and so $V_{Z}^{*} \subseteq \Lambda^{*}(M)$. Thus $V_{Z}^{*}=\Lambda^{*}(M)$ and the lemma is proved.

We also have

LEMMA 7.10. The following conditions hold:

(i) If $K$ is a component of $L_{a}$ of type $L_{2}(16)$ for $a$ in $A^{\#}$, then $K$ lies in a component of $M$ of type $L_{2}(16)$;

(ii) If $J$ is a component of $M$ of type $L_{2}(16)$, then for some $z$ in $Z^{\# \text {, }}$ $L_{2}$ contains a component of type $L_{2}(16)$ which covers $J / O(J)$.

Proof. We have $m(A) \geqslant 7$, so $O(K)$ is generated by its subgroups $C_{O(K)}(B)$ with $B$ in $E_{3}(A)$. Now Lemma 7.7 implies that $O(K) \subseteq M$. Furthermore, by (2.37), $A$ leaves $K$ invariant, $A \cap K \in M(K)$, and $A$ induces only inner automorphisms of $K / O(K)$. Hence if we set $A_{0}=C_{A}(K / O(K))$, we have that $A=A_{0} \times(A \cap K)$. Since $m(A \cap K)=4$, it follows that $m\left(A_{0}\right) \geqslant 3$. But then $N_{G}\left(A_{0}\right) \subseteq M$ and as $C_{K}\left(A_{0}\right)$ covers $K / O(K)$ and $O(K) \subseteq M$, we conclude that $K \subseteq M$.

Thus $K \subseteq L_{2^{\prime}}\left(C_{M}(a)\right)$ and so $K \subseteq L_{2^{\prime}}(M)$ by (2.41). Setting $L=$ $L_{2^{\prime}}(M)$, it follows that $K$ is a component of $L_{2}\left(C_{L}(a)\right)$. But by (2.37) and the fact that $A \in M(M), a$ induces an inner automorphism of each component of $L$ of 2-rank at least 4 and now (2.26) yields that $\bar{K}=\bar{J}$ for some component $\bar{J}$ of $\bar{L}$, where $\bar{M}=M / O(M)$. Hence $K \subseteq O(M) J$. But as $J$ is the ultimate term of the derived series of $O(M) J$ and $K$ is perfect, we conclude that $K \subseteq J$. Thus (i) holds.

Next let $J$ be a component of $M$ of type $L_{2}(16)$. To prove (ii), we need only show that for some $z$ in $Z^{\#}, C_{J}(z)$ covers $J / O(J)$. Indeed, if that is the case, then $J_{1}=L_{2^{\prime}}\left(C_{J}(z)\right)$ is a component of $L_{2^{\prime}}\left(C_{M}(z)\right)$. However, as the latter group is $S$-invariant and $S \subseteq C_{G}(z)$, it follows from (2.44) that $J_{1}$ is contained in a component $K$ of $L_{z}$. As in the proof of (2.48) we conclude easily from (2.19(iv)) and (2.25(ii)) that $K$ must also be of type $L_{2}(16)$. But then $K$ covers $J / O(J)$ as $J_{1}$ does and (ii) will hold.

If some element of $S$ induces a field automorphism of $J$ of order 2, then $m(Z \cap J)=2$ and as $m(Z) \geqslant 3$, some $z$ in $Z^{\#}$ must centralize $J / O(J)$. Suppose then that no involution of $S$ acts irregularly on $J$. We shall prove that there is a component $J_{0}$ of $M$ of type $L_{2}(16)$ on which some involution of 
$S$ acts irregularly. But then $m\left(Z \cap J_{0}\right)=2$ and $J_{0} \neq J$, so any $z$ in $\left(Z \cap J_{0}\right)^{\#}$ will centralize $J / O(J)$.

Since we are in the irregular case, there is an $a$ in $A^{\#}$ such that $L_{a}$ has a component $K$ of type $L_{2}(16)$ on which some involution $x$ of $C_{G}(a)$ acts irregularly. By (2.37), $B=A \cap K \in M(K)$ and so we can choose $x$ to normalize $B$, whence $x \in N_{G}(B) \subseteq M$. On the other hand, by (i), $K \subseteq J_{0}$ for some component $J_{0}$ of $M$ of type $L_{2}(16)$. It follows at once that $x$ acts irregularly on $J_{0}$. Since $S$ is a Sylow 2-subgroup of $M$, an involution of $S$ has the same property and the proof of (ii) is complete.

We now let $F$ denote the product of all components of $M$ of type $L_{2}(16)$ and fix this notation.

LEMMA 7.11. For every $x$ in $I(S)$, we have

$$
\Lambda_{x}^{*} \subseteq \Lambda^{*}(M) \Lambda\left(C_{F_{x}}(x)\right) \text { and } \Lambda\left(C_{F_{x}}(x)\right) \subseteq O(M) \Lambda_{x}^{*},
$$

where $F_{x}$ denotes a suitable product of components of $F$ on which $x$ acts irregularly.

Proof. Set $B=C_{A}(x)$, so that $m(B) \geqslant 4$ as $m(A)=m(S) \geqslant 7$. By $\Lambda^{*}$-generation, $\Lambda_{x}^{*}$ is generated by its subgroups

$$
U_{b}=\Lambda^{*}\left(\Lambda_{x}^{*} \cap C_{G}(b)\right)
$$

for $b$ in $B^{\#}$. Moreover, by (2.46), we have

$$
U_{b} \subseteq \Lambda_{b}^{*} \Lambda\left(C_{J_{b}}(x)\right)
$$

where $J_{b}$ is a suitable product of components of $L_{b}$ of type $L_{2}(16)$ on each of which $x$ acts irregularly and $\Lambda\left(C_{J_{b}}(x)\right) \subseteq \Lambda_{x}^{*}$.

Now each $\Lambda_{b}^{*} \subseteq V_{A}^{*}=\Lambda^{*}(M)$. Hence if we set

$$
J=\left\langle\Lambda\left(C_{J_{b}}(x)\right) \mid b \in B^{\#}\right\rangle,
$$

we obtain that

$$
\Lambda_{x}^{*} \subseteq \Lambda^{*}(M) J \text { and } J \subseteq \Lambda_{x}^{*}
$$

Now by the preceding lemma, each component of each $J_{b}$ lies in a unique component of $F$. We let $F_{x}$ be the product of all such components. Setting $\bar{M}=M / O(M)$, each $\bar{J}_{b}$ is then a product of components of $\bar{F}_{x}$ and $\bar{F}_{x}$ is generated by the $\bar{J}_{b}$ for $b$ in $B^{\#}$. Thus $\bar{x}$ acts irregularly on each component 
of $\bar{F}_{x}$ and it follows that

$$
\Lambda\left(C_{\bar{F}_{x}}(x)\right)=\left\langle\Lambda\left(C_{\bar{J}_{b}}(\bar{x})\right) \mid b \in B^{\#}\right\rangle=\bar{J}
$$

Hence if we set $F_{0}=\Lambda\left(C_{F_{x}}(x)\right)$, we have that $J \subseteq O(M) F_{0}$, whence $J \subseteq$ $C_{O(M)}(x) F_{0}$. However, by definition of $F_{0}, F_{0} \triangleleft C_{O(M)}(x) F_{0}$. Since the factor group modulo $F_{0}$ had odd order and since $J$ is perfect, we must have that $J \subseteq F_{0}$. Thus $\Lambda_{x}^{*} \subseteq \Lambda^{*}(M) J \subseteq \Lambda^{*}(M) F_{0}$, which established the first inclusion. But as $\bar{F}_{0}=\bar{J}$, we also have $\bar{F}_{0} \subseteq O(M) J \subseteq O(M) \Lambda_{x}^{*}$, so the second inclusion holds as well.

The preceding lemma enables us to prove

LEMмA 7.12. If $B \in E(S)$ with $Z \subseteq B$, then

$$
\Lambda^{*}\left(V_{B}^{*}\right)=\Lambda^{*}(M)=V_{A}^{*}
$$

Proof. For $b$ in $B^{\#}$, let $F_{b}$ be the corresponding product of components of $F$ described in Lemma 7.11 and set

$$
F_{B}=\left\langle\Lambda\left(C_{F_{b}}(b)\right) \mid b \in B^{\#}\right\rangle \text {. }
$$

Then the preceding lemma and the definition of $V_{B}^{*}$ yields that

$$
V_{B}^{*} \subseteq \Lambda^{*}(M) F_{B} \text { and } F_{B} \subseteq O(M) V_{B}^{*}
$$

But as $Z \subseteq B, V_{B}^{*} \supseteq V_{Z}^{*}=V_{A}^{*}=\Lambda^{*}(M)$. Since $O(M) \subseteq \Lambda^{*}(M)$, it follows that also $F_{B} \subseteq V_{B}^{*}$ and we conclude that

$$
V_{B}^{*}=\Lambda^{*}(M) F_{B}
$$

Since $\Lambda^{*}(M)$ and $F$ are normal in $M$ with $F \cap \Lambda^{*}(M) \subseteq O(M)$ and $F_{B} \subseteq F$, it follows immediately from the definition of $\Lambda^{*}$ that

$$
\Lambda^{*}\left(V_{B}^{*}\right)=\Lambda^{*}\left(\Lambda^{*}(M)\right) \Lambda^{*}\left(F_{B}\right)=\Lambda^{*}(M) \Lambda^{*}\left(F_{B}\right)
$$

Hence to complete the proof, it will suffice to prove that

$$
\Lambda^{*}\left(F_{B}\right)=O\left(F_{B}\right) \subseteq O(M)
$$

Set $F_{B}^{*}=\left\langle F_{b} \mid b \in B^{\#}\right\rangle$, so that $F_{B}^{*}$ is a product of components of $F$ each of type $L_{2}(16)$. By definition of $\Lambda^{*}$, obviously $\Lambda^{*}\left(F_{B}^{*}\right)=O\left(F_{B}^{*}\right)$. But $F_{B} \subseteq F_{B}^{*}$ and so to prove the desired assertion, we need only show that, in fact, $F_{B}$ covers $F_{B}^{*} / O\left(F_{B}^{*}\right)$, for then also $\Lambda^{*}\left(F_{B}\right)=O\left(F_{B}\right)$. 
Let $K$ be a component of $F_{b}$ for some $b$ in $B^{\#}$, so that $K$ is of type $L_{2}(16)$ and $b$ acts irregularly on $K$. Moreover, we have $\Lambda\left(C_{K}(b)\right) \subseteq F_{B}$. On the other hand, as usual, $Z(S) \cap K=Z \cap K \neq 1$. Choose $z$ in $(Z \cap K)^{\#}$. Since $Z \subseteq B, b_{1}=z b \in B^{\#}$. It will suffice to show that $K$ is necessarily a component of $F_{b_{1}}$. Indeed, if this is the case, then also $\Lambda\left(C_{K}\left(b_{1}\right)\right) \subseteq F_{B}$. But by (2.36(iv))

$$
\left\langle\Lambda\left(C_{K}(b)\right), \Lambda\left(C_{K}\left(b_{1}\right)\right)\right\rangle \text { covers } K / O(K)
$$

and so it will follow that $F_{B}$ covers $K / O(K)$. Since this argument will apply to each such $K$ and each $b$ in $B^{\#}$, we shall then conclude that $F_{B}$ covers $F_{B}^{*} / O\left(F_{B}^{*}\right)$, as required.

By Lemma 7.10 , for some $z_{1}$ in $Z^{\#}, L_{z_{1}}$ has a component $K_{1}$ of type $L_{2}(16)$ with $K_{1} \subseteq K$. Then $b_{1}$ acts irregularly on $K_{1}$ and $\Lambda\left(C_{K_{1}}\left(b_{1}\right)\right)$ covers $\Lambda\left(C_{K}\left(b_{1}\right)\right)$ modulo its core. But now if $\Lambda\left(C_{K_{1}}\left(b_{1}\right)\right) \subseteq \Lambda_{b_{1}}$, it will follow from the preceding lemma that $\Lambda\left(C_{K_{1}}\left(b_{1}\right)\right)$ lies in a component of $F$, which must necessarily be $K$ (as $\Lambda\left(C_{K_{1}}\left(b_{1}\right)\right)$ covers $\Lambda\left(C_{K}\left(b_{1}\right)\right)$ modulo its core). But by (2.46), either this is indeed the case or else $\Lambda\left(C_{K_{1}}\left(b_{1}\right)\right)$ lies in a component $J$ of $L_{b_{1}}$ of type $L_{2}(16)$ on which $z_{1}$ acts irregularly. Thus it remains only to exclude the latter possibility.

Set $E=C_{A}\left(b_{1}\right)$, so that $m(E) \geqslant 4$. As usual, if $P$ is a Sylow 2-subgroup of $H=C_{G}\left(b_{1}\right)$ containing $E$, then $P^{\prime} \cap J \subseteq Z(P) \cap J \neq 1$ and so $E$ leaves

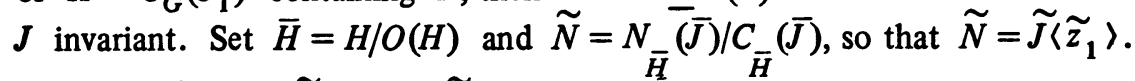
Then $C_{\widetilde{N}}\left(\tilde{z}_{1}\right)=\left\langle\tilde{z}_{1}\right\rangle \times C_{\widetilde{J}}\left(\tilde{z}_{1}\right)$ with $C_{\widetilde{J}}\left(\widetilde{z}_{1}\right) \cong L_{2}(5)$ and consequently $m\left(C_{\widetilde{N}}\left(\widetilde{\tilde{z}}_{1}\right)\right)=3$. But $z_{1} \in Z \subseteq E$ and so $m(\widetilde{E}) \leqslant 3$. We therefore conclude that $C_{\bar{E}}(\bar{J}) \neq 1$. Choose $a$ in $A^{\#}$ so that $\bar{a} \in C_{\bar{E}}(\bar{J})$, in which case $J_{1}=$ $L_{2^{\prime}}\left(C_{J}(a)\right)$ covers $\bar{J}$. In particular, $z_{1}$ also induces a field automorphism of $J_{1}$ of order 2.

Finally we argue as in Lemma 7.4. By (2.43), the normal closure $L$ of $J_{1}$ in $C_{G}(a)$ consists of either one component of type $L_{2}(16)$ or $L_{2}\left(2^{8}\right)$ or of two components of type $L_{2}(16)$ interchanged by $b_{1}$. But $A \subseteq C_{G}(a)$ and so by (2.37), $A \cap L$ is a Sylow 2-subgroup of $L$. In particular, $z_{1}$ centralizes $A \cap L$ and so $z_{1}$ induces an inner automorphism of each component of $L$. However, $C_{J_{1}}\left(z_{1}\right)$ is of type $L_{2}(5)$ and $C_{J_{1}}\left(z_{1}\right) \subseteq C_{L}\left(z_{1}\right)$. We conclude easily from this that $z_{1}$ must centralize $L / O(L)$. But then $z_{1}$ centralizes $J_{1} / O\left(J_{1}\right)$, contrary to the fact that $z_{1}$ induces a field automorphism of $J_{1}$ of order 2. This completes the proof.

This result has two important consequences: 
Lemma 7.13. If $B \in E(S)$ and $Z \subseteq B$, then $N_{G}(B) \subseteq M$.

Proof. For $E$ in $E(G)$, set $Y_{E}=\Lambda^{*}\left(V_{E}^{*}\right)$. It is immediate from the definition that for $x$ in $G,\left(Y_{E}\right)^{x}=Y_{E^{x}}$. In particular, if $B$ is as in the lemma and $x \in N_{G}(B)$, it follows that $\left(Y_{B}\right)^{x}=Y_{B}$. However, by the preceding lemma, $Y_{B}=\Lambda^{*}(M)=V_{A}^{*}$. Thus $x \in M=N_{G}\left(V_{A}^{*}\right)$ and so $N_{G}(B) \subseteq M$.

As customary, we say that $M$ controls fusion in $S$ if two subsets of $S$ conjugate in $G$ are conjugate in $M$. We now prove

LEMMA 7.14. $M$ controls fusion in $S$.

Proof. By (2.70), we know that all fusion in $S$ is determined by the groups $N_{G}(D)$ as $D$ ranges over the elements of $D$. Hence to prove the lemma, we need only show that each such $N_{G}(D) \subseteq M$. However, $D \triangleleft S$ and $C_{S}(D)$ $\subseteq D$. Thus $Z(S) \subseteq D$ and so if we set $B=\Omega_{1}(Z(D))$, it follows that $Z \subseteq B$. Now the preceding lemma yields that $N_{G}(B) \subseteq M$. Since clearly $N_{G}(D) \subseteq$ $N_{G}(B)$, the lemma follows.

Now we can eliminate the irregular case.

LEMMA 7.15. The irregular case does not occur.

Proof. By Lemma 7.10, $F$ is nontrivial. Setting $E=S \cap F$, we have that $E$ is elementary abelian and that $E \in S(F)$. But $F \triangleleft M$ and so any conjugate $x^{\prime}$ in $M$ of an element $x$ of $E$ necessarily lies in $F$. In particular, if $x^{\prime} \in S$, then $x^{\prime} \in E$. Thus $E$ is strongly closed in $S$ with respect to $M$. Now the preceding lemma yields that $E$ is strongly closed in $S$ with respect to $G$, contrary to Proposition 3.1.

We have therefore obtained our objective:

LEMMA 7.16. We have $\Gamma_{S, 3}(G) \subseteq M$.

We next prove

LEMMA 7.17. $M$ controls fusion in $S$.

Proof. As in Lemma 7.14, we need only show that $N_{G}(D) \subseteq M$ for each $D$ in $D$, which will follow from the preceding lemma if each $m(D) \geqslant 3$. Suppose false for some $D$ in $D$. Since $m(S) \geqslant 5$, this implies that $m(S / D) \geqslant 3$. But now (2.66) forces $m(D) \geqslant 6$, contrary to our assumption.

This in turn implies

LEMMA 7.18. The following conditions hold:

(i) $O(M)=O_{2^{\prime}, 2}(M)$;

(ii) No component of $M$ has abelian Sylow 2-subgroups. 
Proof. Set $P=S \cap O_{2^{\prime}, 2}(M)$. If $P \neq 1$, then it is immediate that $Z(P)$ is a nontrivial abelian subgroup of $S$ which is strongly closed in $S$ with respect to $M$. But then $Z(P)$ is strongly closed with respect to $G$ by the preceding lemma, contrary to Proposition 3.1. Thus $P=1$ and so (i) holds. Likewise if $M$ has a component with abelian Sylow 2-subgroups, it follows as in Lemmas 7.15 that $S$ has a nontrivial abelian subgroup that is strongly closed in $S$ with respect to $G$, giving the same contradiction.

As a corollary we have

LEMMA 7.19. Every component of $\Lambda(M)$ is of type $L_{2}(q)$ for some $q \equiv$ 7, $9(\bmod 16)$ or of type $A_{7}$.

We can now pin things down rather tightly.

LEMMA 7.20. The following conditions hold:

(i) $\Lambda(M)$ has $m \geqslant 3$ components;

(ii) $\Lambda(M)=L_{2},(M)$;

(iii) $S=T_{1} \times T_{2} \times \cdots \times T_{m} \times B$, where $T_{i} \cong D_{8}, 1 \leqslant i \leqslant m$, and $B$ is elementary of rank at most $m$;

(iv) Every involution of $G$ is central.

Proof. As in the first paragraph of the proof of Lemma 7.9, we have that $V_{A}^{*} \subseteq \Lambda^{*}(M)$. It follows therefore from Lemma 7.2 that $J=\Lambda(M) \neq 1$. Suppose $J \subset L_{2}(M)$ and let $K$ be the product of all components of $M$ of even type, so that $L_{2}(M)=J K$. Set $\bar{M}=M / O(M)$, so that $\bar{K} \triangleleft \bar{M}$. By Lemma 7.18(ii), every component of $\bar{K}$ is simple and has nonabelian Sylow 2-subgroups. It follows therefore from (2.13) and (2.18) that every element of $\bar{S}$ induces an inner automorphism of $\bar{K}$. Hence we can write $S=S_{1} \times S_{2}$, where $S_{1}=S \cap$ $K$ and $S_{2}=C_{S}(\bar{K})$. Furthermore, $\bar{S}_{2}$ is a Sylow 2-subgroup of $\bar{C}=C_{\bar{M}}(\bar{K})$. We have that $\bar{C} \triangleleft \bar{M}$ as $\bar{K} \triangleleft \bar{M}$ and that $\bar{C} \cap \bar{K}=1$. Together these conditions imply that $S_{1}$ and $S_{2}$ are each strongly closed in $S$ with respect to $M$. By Lemma 7.17, $S_{1}$ and $S_{2}$ are thus strongly closed in $S$ with respect to $G$. However, as $S_{1} \neq 1$ and $S_{2} \neq 1$, this contradicts (2.73). Hence $J=L_{2}$ (M) and (ii) holds.

Let $m$ be the number of components of $J$ and suppose now that $m \leqslant 2$. By Lemma 7.18(i), $O_{2}(\bar{M})=1$ and as $\bar{J}=L(\bar{M}),(2.8)$ implies that $C_{\bar{M}}(\bar{J})=1$. Thus $\bar{S}$ acts faithfully on $\bar{J}$. However, as each component of $\bar{J}$ has dihedral Sylow 2-subgroups, we have $m(\bar{J}) \leqslant 4$. But $m(\bar{S}) \geqslant 5$. Hence considering the structure of Aut $(\bar{J})$, we see that $\bar{M}$ must have a normal subgroup of index 2 and we conclude from Lemma 7.17 that $G$ does as well, contrary to the simplicity of $G$. Hence (i) holds. 
Now let $J_{i}, 1 \leqslant i \leqslant m$, be the components of $J$ and set $T_{i}=S \cap J_{i}$. Then each $T_{i} \cong D_{8}$ and so $S$ leaves each $J_{i}$ invariant by (2.13). By (2.18), the image $\widetilde{S}_{i}$ of $\bar{S}$ in $\widetilde{N}_{i}=N_{\bar{M}}\left(\bar{J}_{i}\right) / C_{\bar{M}}\left(\bar{J}_{i}\right)$ is isomorphic to $D_{8}$ or $D_{8} \times E_{2}$, $1 \leqslant i \leqslant m$. From this and the fact that $C_{\bar{M}}(\bar{J})=1$, we easily deduce that $S=$ $T \times B$, where $T$ is the product of the $T_{i}, 1 \leqslant i \leqslant m$, and $B$ is elementary of rank at most $m$. Thus (iii) also holds.

Finally we have $Z(S)=Z(T) \times B$ with $Z(T)$ the direct product of the groups $Z\left(T_{i}\right) \cong E_{2}, 1 \leqslant i \leqslant m$. We have ${\widetilde{S_{i}}}_{i} \widetilde{T}_{i} \times \widetilde{B}_{i}$, where $\widetilde{B}_{i}$ is the image of $B$ in $\widetilde{N}_{i}, 1 \leqslant i \leqslant m$. By (2.31), every involution $\widetilde{S}_{i}$ is conjugate under the action of $\widetilde{J}_{i}$ to an element of $Z\left(\widetilde{T}_{i}\right) \widetilde{B}_{i}, 1 \leqslant i \leqslant m$. From this, it follows at once that every involution of $S$ is conjugate in $M$ to an element of $Z(T) B=Z(S)$. Hence every involution of $G$ is conjugate to an element of $Z(S)$ and so is a central involution. Thus (iv) also holds and the lemma is proved.

We are finally in a position to prove that $M$ is strongly embedded in $G$. We argue by contradiction. We have that $M \subset G$ and as $\Gamma_{S, 3}(G) \subseteq M$, also $N_{G}(S) \subseteq M$. Hence $H=C_{G}(x) \nsubseteq M$ for some $x$ in $I(S)$. Since $M$ controls fusion in $S$ and since $x$ is conjugate in $M$ to an element of $Z(S)$, we can assume without loss that $x \in Z(S)$. In particular, $x \in A$ and so $\Lambda^{*}(H)=\Lambda_{x}^{*}$ $\subseteq \Lambda_{A}^{*} \subseteq M$.

Suppose $\Lambda(H) \neq 1$. Since no component of $\Lambda(H)$ is of type $\operatorname{SL}(2, q)$, $q$ odd, $m(\Lambda(H)) \geqslant 2$ and $x \notin \Lambda(H)$. Hence if we set $Q=(S \cap \Lambda(H))\langle x\rangle$, we have that $m(Q) \geqslant 3$. Moreover, by the Frattini argument, $H=\Lambda^{*}(H) N_{H}(Q)$. Since $N_{H}(Q) \subseteq \Gamma_{S, 3}(G) \subseteq M$, we conclude that $H \subseteq M$, contrary to our choice of $H$. Thus $\Lambda(H)=1$.

Next set $K=L_{2},(H)$ and $\bar{H}=H / O(H)$. Suppose $K=1$ in which case $H$ is 2-constrained. Setting $R=S \cap O_{2^{\prime}}(H)$, we have $\bar{R}=O_{2}(\bar{H})$. Since $m(\bar{S}) \geqslant 5$, application of $(2.8)$ forces $m(\bar{R}) \geqslant 3$. But then $H=O(H) N_{N}(R)$ and $N_{H}(R) \subseteq \Gamma_{S, 3} \subseteq M$. Since $O(H) \subseteq \Lambda^{*}(H) \subseteq M$, again we reach the contradiction $H \subseteq M$. Thus $K \neq 1$.

Similarly if $K_{1}$ is a component of $K$, consideration of $\left(S \cap \bar{K}_{1}\right)\langle\bar{x}\rangle$ and its normalizer yields that $M$ covers all components of $\bar{K}$ other than $\bar{K}_{1}$. But then if $K$ has more than one component it follows that $M$ covers $\bar{K}$, whence $K \subseteq M$. Furthermore, if we set $P=S \cap K$, we have $H=O(H) K N_{H}(P)$ by the Frattini argument with $m(P) \geqslant 3$ and again we reach the contradiction $H \subseteq M$. We conclude that $K=L_{2}(H)$ consists of a single component of even type. Likewise it follows that $K \nsubseteq M$, otherwise we reach the same contradiction by another application of the Frattini argument. Hence $M$ does not cover $\bar{K}$.

Suppose next that $\bar{K} \cong L_{2}(9)$ or $\operatorname{Psp}\left(4,2^{n}\right)$, or that $\bar{K} / Z(\bar{K}) \cong L_{3}\left(2^{n}\right)$, $n \geqslant 2$. We conclude at once from $\left(2.36\left(\right.\right.$ iii)) that $\bar{K} \subseteq \Gamma_{\bar{S}, 3}(\bar{H})$ and consequently 
$M$ covers $\bar{K}$, a contradiction. Hence $\bar{K} \cong L_{2}\left(2^{n}\right), S z\left(2^{n}\right)$, or $U_{3}\left(2^{n}\right)$.

In the latter two cases $S=P \times C_{S}(\bar{K})$ by (2.19(iii)), whence $S \neq \Omega_{1}(S)$. But $S=\Omega_{1}(S)$ by $\left(7.20\left(\right.\right.$ iii)), and thus we conclude that $\bar{K} \cong L_{2}\left(2^{n}\right), n \geqslant 3$ (as $K$ is of even type).

Finally by (2.19(i)), we can write $S=P U$ with $P \cap U=1$ and $\left|U: C_{S}(\bar{K})\right| \leqslant 2$, with equality holding only if some element of $S$ induces a nontrivial outer automorphism of $\bar{K}$. By (2.36(iv)) we have

$$
\bar{K}=\left\langle N_{\bar{K}}(\bar{P}), C_{\bar{K}}(\bar{U})\right\rangle .
$$

If $m(U) \geqslant 3$, we see that $\bar{K} \subseteq \Gamma_{\bar{S}, 3}(\bar{H})$, so $M$ covers $\bar{K}$, which is not the case. Thus $m(U) \leqslant 2$. Note also that by the structure of $S$, we have $S=\Omega_{1}(S)$. Since $U \cong S / P$, it follows that also $U=\Omega_{1}(U)$. To eliminate this case, it will suffice to prove that $C_{Z(S)}(\bar{K})$ is noncyclic. Indeed, as $U=\Omega_{1}(U)$ with $m(U) \leqslant 2$ and $U \supseteq C_{S}(\bar{K})$, this will force $U=C_{Z(S)}(\bar{K}) \cong E_{4}$, whence $S=$ $P \times U$ is elementary, which is not the case.

Set $\widetilde{S}=S / C_{S}(K)=\widetilde{P} \widetilde{U}$, so that $\widetilde{P}$ is elementary of index at most 2 in $\widetilde{S}$. We consider the product $T$ of the dihedral groups $T_{i}, 1 \leqslant i \leqslant m$. Then $\widetilde{T}$ has an elementary subgroup of index at most 2 . However, it is immediate from the structure of $T$ that this is possible only if $Z(T) \cap C_{S}(\bar{K})$ has index at most 2 in $Z(T)$. However, $Z(T) \cong E_{2^{m}}$ with $m \geqslant 3$ and $Z(T) \subseteq Z(S)$. Together these conditions yield the desired conclusion that $C_{Z(S)}(K)$ is noncyclic. This completes the proof of Proposition 7.1.

8. The even $A$-hull. By Proposition 7.1, we now know that $L_{1}(G)$ is either empty or consists of an evenly embedded $L_{2}(5)$. Hence Proposition 3.2 is applicable and yields

$$
O\left(C_{G}(x)\right)=1 \text { for all } x \text { in } I(G) .
$$

As a consequence, we have

$$
L_{x}=L_{2}\left(C_{G}(x)\right)=L\left(C_{G}(x)\right) \text { for all } x \text { in } I(G) .
$$

Moreover, the components of $L_{x}$ are either elements of $\hat{A}_{2}$ or isomorphic to $L_{2}(5)$.

For any subgroup $Q$ of $G$, we now set

$$
X_{Q}=\left\langle L_{x} \mid x \in I(Q)\right\rangle=\left\langle L\left(C_{G}(x)\right) \mid x \in I(Q)\right\rangle .
$$

We call $X_{Q}$ the even $Q$-hull (inasmuch as all components of each $L_{x}$ are of even type or isomorphic to $\left.L_{2}(5)=L_{2}(4)\right)$.

By assumption, $L(G)$ is nonempty. We now define 


$$
r=\max \{m(K / Z(K)) \mid K \in L(G)\} \text {. }
$$

We next choose $A$ in $N(G)$ such that

(a) $L(G ; A)$ contains an element $K$ with $m(K / Z(K))=r$;

(b) Subject to (a), $m(A)$ is maximal.

Recall that $L(G ; A)$ is the set of components of $C_{G}(a)$ as $a$ ranges over $A^{\#}$. Note that in all cases, $K$ is a component of $L_{a}$ for some $a$ in $A^{\#}$ and so $X_{A} \neq 1$.

Our goal in this section is to prove

Proposition 8.1. For some choice of $A$, the following conditions hold:

(i) The even A-hull $X_{A}$ is semisimple;

(ii) The components of $X_{A}$ are elements of $L(G ; A)$ and include $K$;

(iii) Every element of $L(G ; A)$ either lies in $K$ or centralizes $K$;

(iv) $A \in M(G)$.

We argue by contradiction in a long sequence of lemmas. We first consider an arbitrary element $A$ of $N(G)$ satisfying conditions (a) and (b) above. We fix $K$ in $L(G ; A)$, as above, and we set $B=C_{A}(K)$. Since $K$ is a component of some $L_{a}$ with $a$ in $A^{\#}$, we have $a \in B$ and so $B \neq 1$.

LemMA 8.2. For each $b$ in $B^{\#}, K$ is a component of $L_{b}$.

Proof. Let $b \in B^{\#}$. By $L$-balance and (2.43) the normal closure $J$ of $K$ in $L_{b}$ consists of either a single component or two components interchanged by $a$ and $K=L\left(C_{J}(a)\right.$ ) (using the fact that $O\left(L_{b}\right)=1$ ). If $L$ consists of a single component, our maximal choice of $K$ forces $m(J / Z(J))=r$. But then (2.26) implies that $K=J$, whence $K$ is a component of $L_{b}$. In particular, this will be the case if either $A \in M\left(C_{G}(b)\right)$ or $J$ has nonabelian Sylow 2-subgroups by (2.43(iii)).

In the remaining case, $J$ consists of two components $J_{1}$ and $J_{2}$ with abelian Sylow 2-subgroups and if $A^{*} \in M\left(C_{G}(b)\right)$, then $m\left(A^{*}\right)>m(A)$. Furthermore, by (2.12), we have $J_{1} \cong K$. On the other hand, by (2.37(ii)) $A^{*}$ $\cap J_{2} \neq 1$. Choose $a^{*}$ in $\left(A^{*} \cap J_{2}\right)^{\#}$. Again by $L$-balance and (2.43), the normal closure $F$ of $J_{1}$ in $L_{a}$ * consists of either a single component or two components interchanged by $b$ and $J_{1}=L\left(C_{F}(b)\right)$. Since $J_{1} \cong K$, it follows as in the preceding paragraph from our maximal choice of $K$ that a component $F_{1}$ (possibly $F$ itself) of $C_{F}(b)$ is isomorphic to $J_{1}$ and hence to $K$. But $F_{1} \in L\left(G ; A^{*}\right)$ and $m\left(A^{*}\right)>m(A)$, so our maximal choice of $A$ is contradicted. Hence this case cannot arise and the lemma is proved.

Taking $b=a$ and arguing in essentially the same way, we easily obtain

Lemma 8.3. $K$ is A-invariant. 
As a further consequence, we have

Lemma 8.4. $X_{B}$ normalizes $K$.

Proof. This is immediate as $L_{b}$ normalizes $K$ for each $b$ in $B^{\#}$ by Lemma 8.2.

We next prove

\section{Lemma 8.5. $X_{A}$ does not normalize $K$.}

Proof. Suppose false, in which case $X_{A} \subseteq N=N_{G}(K)$. Let $y \in A^{\#}$. We shall argue now that $L_{y}=L_{1} L_{2}$, where $L_{1}$ is the product of the components of $L_{y}$ which lie in $K$ and $L_{2}$ is the product of those which centralize $K$. Since $L_{y} \subseteq X_{A} \subseteq N, L_{y} \subseteq L_{2^{\prime}}\left(C_{N}(y)\right)$, whence $L_{y} \subseteq J=L_{2^{\prime}}(N)$ by (2.41). Clearly $K$ is a component of $J$ and so $J=K J_{0}$, where $J_{0}$ is the product of the remaining components of $J$. Since $K$ is $A$-invariant, so also is $J_{0}$ and therefore $y$ leaves both $K$ and $J_{0}$ invariant and we also have $L_{y} \triangleleft L_{2^{\prime}}\left(C_{J}(y)\right)$.

Setting $\bar{N}=N / Z(K)$, we have that $\bar{K} \cap \bar{J}_{0}=1$, whence $\bar{J}=\bar{K} \times \bar{J}_{0}$. Since $\bar{y}$ leaves each factor invariant, it follows that $C_{\bar{J}}(\bar{y})=C_{\bar{K}}(\bar{y}) \times C_{\bar{J}_{0}}(\bar{y})$. Since $Z(K)$ is a 2-group in the center of $J$, this implies that $O^{2}\left(C_{J}(y)\right)=$ $O^{2}\left(C_{K}(y)\right) O^{2}\left(C_{J_{0}}(y)\right)$, whence $J_{y}=L_{2^{\prime}}\left(C_{J}(y)\right)=L_{2^{\prime}}\left(C_{K}(y)\right) L_{2^{\prime}}\left(C_{J_{0}}(y)\right)$. Thus every component of $J_{y}$ either lies in $K$ or centralizes $K$. Since $L_{y} \triangleleft$ $J_{y}$, the desired factorization of $L_{y}$ now follows.

On the other hand, as $K$ is $A$-invariant and $A \in N(G)$, some element $x$ of $A^{\#}$ lies in $K$. Then $x$ centralizes $L_{2}$ and as $L_{2}$ is a product of components of $L_{y}$, we conclude by $L$-balance that $L_{2} \subseteq L_{x}$. This holds for each $y$ in $A^{\#}$ and consequently $X_{A} \subseteq K L_{x}$. But $K \subseteq L_{a} \subseteq X_{A}$ and so $X_{A}=K L_{x}$. If $M$ denotes the product of the components of $L_{x}$ which centralize $K$, our argument yields that $K L_{x}=K M$. Thus $X_{A}$ is a semisimple group, whose components are elements of $L(G ; A)$ and include $K$. Moreover, our argument shows that every element of $L(G ; A)$ either lies in $K$ or centralizes $K$.

Finally let $S$ be a Sylow 2-subgroup of $G$ containing $A$. It is immediate from the definition of $X_{A}$ that $S$ normalizes $X_{A}$. Let $A^{*} \in M(S)$. Then $A^{*}$ leaves $K$ invariant by (2.37). Since $A \triangleleft S$, it follows that $A^{*}$ leaves $B=$ $C_{A}(K)$ invariant. But $B \neq 1$ and so $C_{B}\left(A^{*}\right) \neq 1$. Since $A^{*} \in M(S)$, we conclude that $b \in A^{*}$ for some $b$ in $B^{\#}$. Since $K$ is a component of $L_{b}$ by Lemma 8.2, we see that $K \in L\left(G ; A^{*}\right)$. Now our maximal choice of $A$ forces $m(A)=m\left(A^{*}\right)$, whence $A \in M(G)$.

We have thus shown that all parts of Proposition 8.1 hold. Since we are arguing by contradiction, we accept this lemma.

LEMMA 8.6. One of the following holds: 
(i) $r \geqslant 3$ and $m(G) \leqslant 2 r$; or

(ii) $r=2, m(G) \leqslant 6$, and some element of $L(G ; A)$ is isomorphic to $L_{2}(9)$.

Proof. If $m(G) \leqslant 2 r$, then necessarily $r \geqslant 3$ as $m(G) \geqslant 5$ by Proposition 5.14. Thus (i) holds in this case. Hence we can suppose $m(G)>2 r$. We shall argue that either $A \in M(G)$ or (ii) holds. Choose $A^{*}$ in $M(G)$, so that $m\left(A^{*}\right) \geqslant 2 r+1$. Setting $E=C_{A^{*}}(a)$, we have that $m(E) \geqslant r+1$. Moreover, $m(E) \geqslant 4$ if $m\left(A^{*}\right) \geqslant 7$. Now $E \subseteq C_{G}(a)$ and so $E$ acts on $L_{a}$. Consider first the case that $E$ leaves $K$ invariant. If $K \neq L_{2}(9)$ or $m(E) \geqslant 4$, it follows from (2.23(iii), (iv)) that $C_{E}(K) \neq 1$. In these cases choose $x$ in $E^{\#}$ to centralize $K$. As usual, $L$-balance together with our maximal choice of $K$ implies then that $L_{x}$ possesses a component $L$ with $m(L / Z(L))=r$. Since $x \in A^{*}, L \in L\left(G ; A^{*}\right)$ and our maximal choice of $A$ forces $m(A)=m\left(A^{*}\right)$, whence $A \in M(G)$. This argument applies unless $m(E) \leqslant 3$ and $K \cong L_{2}(9)$, whence also $m\left(A^{*}\right)=m(G) \leqslant 6$ and $r=m(K)=2$, precisely the conditions of (ii).

On the other hand, if $E$ does not leave $K$ invariant, then $K$ has abelian Sylow 2-subgroups and for some $x$ in $E^{\#}$, we have $K \neq K^{x}$ and $L\left(C_{K K^{x}}(x) \cong K\right.$. Again $L$-balance and the maximality of $K$ forces $A$ to be an element of $M(G)$. This proves our assertion.

We can therefore assume that $A \in M(G)$, whence $m(A) \geqslant 2 r+1$. We know that $A$ leaves $K$ invariant and now (2.23) yields that either $m(B) \geqslant r+1$ or $K \cong$ $L_{2}(9)$ and $m(A) \leqslant 6$. Since (ii) holds in the latter case, we can suppose that $m(B)$ $\geqslant r+1$.

Finally as $A \in M(G), A$ acts on each component $J$ of $L_{y}$ for any $y$ in $A^{\#}$. Moreover, our maximal choice of $K$ implies that $m(J / Z(J)) \leqslant r$. Since $m(B) \geqslant$ $r+1$, we conclude from (2.23) that

$$
L_{y}=\left\langle L\left(C_{L_{y}}(b)\right) \mid b \in B^{\#}\right\rangle
$$

if either $m(B) \geqslant 4$ (in particular, if $r \geqslant 3$ ) or if no component of $L_{y}$ is isomorphic to $L_{2}(9)$. In the exceptional case we have $r=2, m(A) \leqslant 6$, and some component of $L_{y}$ is isomorphic to $L_{2}(9)$, so (ii) holds. Hence we can assume that we have the given generation of $L_{y}$ for each $y$ in $A^{\#}$. But then by $L$-balance, we obtain that $L_{y} \subseteq X_{B}$ for $y$ in $A^{\#}$, whence $X_{A} \subseteq X_{B}$. Thus $X_{A}=X_{B}$ as $B \subseteq A$. However, this is impossible as $X_{B}$ normalizes $K$, while $X_{A}$ does not, by Lemmas 8.4 and 8.5 .

We now sharpen the preceding result.

Lemma 8.7. We have $r \geqslant 3$ and $m(G) \leqslant 2 r$. 
Proof. By the preceding lemma, we need only derive a contradiction from the assumption that $r=2, m(G) \leqslant 6$, and some element of $L(G ; A)$ is isomorphic to $L_{2}(9)$. By Proposition 5.14, we have $m(G) \geqslant 5$ and so $m(G)=5$ or 6. Hence by Proposition 5.2(ii) there exists $z$ in $I(G)$ with $m\left(C_{G}(z)\right)=$ $m(G)$ such that $L_{z}$ has a component of type $L_{2}(9)$. Then $z \in A^{*}$ for some $A^{*}$ in $M(G)$ and now our maximal choice of $A$ forces $m(A)=m\left(A^{*}\right)$. Thus $A \in M(G)$.

Since $r=2$, every element of $L(G ; A)$ is isomorphic to $L_{2}(5), U_{3}(4)$, or $L_{2}(9)$. If possible, we choose $K$ isomorphic to one of the first two groups. We set $B=C_{A}(K)$, so that $m(B)=3$ or 4 if $K \cong L_{2}(5)$ or $U_{3}(4)$ and $m(B)=2,3$, or 4 if $K \cong L_{2}(9)$.

By Lemma $8.5, L_{x}$ does not normalize $K$ for some $x$ in $A^{\#}$ and so some component $J$ of $L_{x}$ does not normalize $K$. We claim that $J \cong L_{2}(9)$ and $m(B)=2$ or 3 . If $J \nRightarrow L_{2}(9)$, then neither is $K$ by our choice of $K$, whence $m(B)=3$ or 4 . But $A$ acts on $J$ as $A \in M(G)$. Since $J \cong L_{2}(5)$ or $U_{3}(4)$, it follows from (2.23) that some $b$ in $B^{\#}$ centralizes $J$. On the other hand, if $m(B)=4$, the same conclusion holds even if $J \cong L_{2}(9)$ by (2.23(iii)). Since $K$ is a component of $L_{b}$ and $J \subseteq L_{b}, J$ thus normalizes $K$, contrary to our choice of $J$. This proves our assertions.

Setting $E=C_{A}(J)$, we reach the same contradiction if $E \cap B \neq 1$, so $E$ $\cap B=1$. Since $m(A / B)=2$ or 3 , this forces $m(E) \leqslant 3$. But $m(E) \geqslant 2$ as also $m(A / E)=2$ or 3 , so $m(E)=2$ or 3 . Furthermore in the latter case $E$ must act faithfully on $K$ as $E \cap B=1$, in which case $K \cong L_{2}(9)$. The situation now is essentially identical to that which we treated in Lemma 6.12. There we had $K$ and $J$ quasisimple with $K / O(K) \cong J / O(J) \cong A_{7}, m(A)=5$, and $m(B)=m(E)=2$; while here we have $K \cong L_{2}(5), U_{3}(4)$ or $L_{2}(9), J \cong L_{2}(9)$ $\cong A_{6}, m(A)=5$ or $6, m(B)=2$ or 3 , and $m(E)=2$ or 3 .

However, we can repeat that proof with essentially no change. There are only three points that should be mentioned. First, if $u$ is an involution of $N_{J}(A)$, we again have that $\left|A: C_{A}(u)\right| \leqslant 2$, so $u$ centralizes some $b$ in $B^{\#}$ and also centralizes some involution of $A \cap K$. Together these conditions force $u$ to lie in $C_{G}(b)$ and to normalize $K$. Secondly in the present case, $L(G)$ is a subset of $\left\{L_{2}(5), U_{3}(4), L_{2}(9)\right\}$, so certainly $K$ is a nonembedded component of $L\left(G ; A^{*}\right)$, where $A^{*}$ has the same meaning as in Lemma 6.12. Thus we again have that $N_{G}(K)$ covers both $N_{\bar{J}}(\bar{A})$ and $N_{\bar{J}}\left(\bar{A}^{*}\right)$, where again $\overline{J A}=J A / O(J) E=J A / E$. In the present case, either $\overline{J A}=\bar{J} \cong L_{2}(9)$ or $\overline{J A} \cong S_{6}$, In either case $\left(2.35\left(\right.\right.$ viii)) implies that $\bar{J}=\left\langle N_{\bar{J}}(\bar{A}), N_{\bar{J}}\left(\bar{A}^{*}\right)\right\rangle$.

Thus $N_{G}(K)$ covers $\bar{J} \cong J$ and so $N_{G}(K) \supseteq J$, contrary to our choice of $J$. As a corollary, we have 
Lemma 8.8. If $b \in B^{\#}$, then $K$ is the only component of its isomorphism type in $L_{b}$. In particular $K \triangleleft C_{G}(b)$ and $K \triangleleft N_{G}(B)$.

Proof. We know that $K$ is a component of $L_{b}$. If $Z(K)=1$, then $K$ is the only component of its isomorphism type in $L_{b}$, otherwise $m\left(L_{b}\langle b\rangle\right) \geqslant$ $2 r+1$, contrary to the preceding lemma. On the other hand, if $Z(K) \neq 1$, then $K / Z(K) \cong L_{3}(4)$ and $r=4$. But then we reach the same conclusion, since otherwise $m\left(L_{b}\right) \geqslant 9 \geqslant 2 r+1$ by (2.16). Thus $K$ is characteristic in $C_{G}(B)$. Hence $C_{G}(b)$ and $N_{G}(B)$ normalize $K$.

We can also prove

Lemma 8.9. We have $A=(A \cap K) B$ with $A \cap K \in M(K)$.

Proof. We know that $K$ is $A$-invariant. Since $r \geqslant 3, K \neq L_{2}(5)$. It follows therefore from (2.37(iii)) that $A \cap K \in M(K)$ and that every element of $A$ induces an inner automorphism of $A$. Hence $A=(A \cap K) C_{A}(K)=(A \cap K) B$.

This completes the preliminary analysis. As in the corresponding analysis of Collin's characterization of the Suzuki groups [4], the main part of the proof consists in establishing the following assertion:

\section{$N_{G}(A)$ normalizes $K$ for some choice of $A$.}

REMARKS. We shall actually prove that except in the case $K \cong P S_{p}\left(4,2^{n}\right)$, $N_{G}(A)$ normalizes $K$ for any choice of $A$.

We shall prove this result in a sequence of lemmas. 'We set $N=N_{G}(A)$ and $A_{1}=A \cap K$. Note that $A=A_{1} B$ with the factorization direct unless $Z(K) \neq 1$, in which case $K / Z(K) \cong L_{3}(4)$. We also set $W=N_{N}(K)$. Our goal is to show for some choice of $A$ that $W=N$ and so we can assume by contradiction that $W \subset N$ for any $A$.

LEMMA 8.10. If $y \in N-W$, then $B \cap B^{y}=1$.

Proof. If not, pick $b$ in $\left(B \cap B^{y}\right)^{\#}$. Then $K$ and $K^{y}$ are components of $L_{b}$ and $K \neq K^{y}$, so $L_{b}$ has more than one component isomorphic to $K$, contrary to what we have shown in Lemma 8.8.

LEMMA 8.11. The following conditions hold:

(i) $W=N_{N}(B)$;

(ii) $W \cap K=N_{K}\left(A_{1}\right)$.

Proof. Since $C_{G}(B) \subseteq C_{G}(a)$ as $a \in B, K$ is a component of $L\left(C_{G}(B)\right)$ and, as above, it is the only component of its isomorphism type, so $K$ is characteristic in $C_{G}(B)$. Hence $N_{G}(B)$ normalizes $K$, whence $N_{N}(B) \subseteq N_{N}(K)$ $\subseteq W$. On the other hand, $W$ normalizes $B=C_{A}(K)$ as it normalizes both $K$ 
and $A$, so $W \subseteq N_{N}(B)$ and (i) follows. Clearly $W \cap K=N_{K}(A)=N_{K}\left(A_{1}\right)$, so (ii) also holds.

Lemmas 8.10 and 8.11 together immediately yield:

Lemma 8.12. Any two distinct conjugates of $B$ in $N$ intersect trivially.

We break up the analysis into two cases according as the Lie rank of $K$ is 1 or 2 .

Case 1. $K \cong L_{2}\left(2^{n}\right), S z\left(2^{n}\right)$, or $U_{3}\left(2^{n}\right), n \geqslant 2$;

Case 2. $K \cong L_{3}\left(2^{n}\right), P S p\left(4,2^{n}\right), n \geqslant 2$, or a covering group of $L_{3}(4)$.

Note that in Case 1 , as $m(K)=r$, we have, in fact, $n=r$.

In Lemmas 8.13-8.22, we assume that Case 1 holds.

Lemma 8.13. If $B^{y} \cap A_{1} \neq 1$ for $y$ in $N$, then $B^{y} \subseteq A_{1}$.

Proof. Set $E=B^{y} \cap A_{1}, m(B)=b$, and $m(E)=e$. We know that $b \leqslant r$ as $m(G) \leqslant 2 r$ and we have $e \leqslant b$. To establish the lemma, we must show that $e=b$.

Setting $W_{1}=W \cap K$, we have $W_{1}=N_{K}\left(A_{1}\right)$ and consequently by the structure of $K, W_{1} / C_{W_{1}}\left(A_{1}\right)$ is cyclic of order $2^{r}-1$ and acts regularly on $A_{1}$. Since $W_{1} \cap W^{y}$ and $C_{W_{1}}\left(A_{1}\right)$ leave $E$ invariant, it follows that $\left[\left(W_{1} \cap W^{y}\right) C_{W_{1}}\left(A_{1}\right)\right] / C_{W_{1}}\left(A_{1}\right)$ has order at most $2^{e}-1$, whence $\left|W_{1}: W_{1} \cap W^{y}\right| \geqslant\left(2^{r}-1\right) /\left(2^{e}-1\right)$. On the other hand, if $u$ and $v$ are representatives of distinct cosets of $W_{1} \cap W^{y}$ in $W_{1}$, Lemma 8.12 implies that $E^{u} \cap E^{v}=1$. Since $\left|A_{1}^{\#}\right|=2^{r}-1$ and $\left|E^{\#}\right|=2^{e}-1$, there are thus at most $\left(2^{r}-1\right) /\left(2^{e}-1\right)$ such cosets and we conclude that

$$
\left|W_{1}: W_{1} \cap W^{y}\right|=\left(2^{r}-1\right) /\left(2^{e}-1\right) .
$$

Since $W_{1}$ centralizes $A / A_{1}$, it leaves $A_{1} B^{y}$ invariant. Since $W^{y}$ leaves $B^{y}$ invariant, the preceding equality shows that $B^{y}$ has precisely $\left(2^{r}-1\right) /\left(2^{e}-1\right)$ distinct conjugates in $A_{1} B^{y}$ under the action of $W_{1}$. Moreover, two such conjugates intersect only in the identity by Lemma 8.12. Hence counting the nonidentity elements in their union, we obtain

$$
\left(2^{b}-1\right) \cdot\left(2^{r}-1\right) /\left(2^{e}-1\right) \leqslant 2^{b+r-e}-1,
$$

the integer on the right representing the number of nonidentity elements in $A_{1} B^{y}$. Simplifying, we obtain

$$
2^{r}+2^{b} \geqslant 2^{b+r-e}+2^{e} .
$$

Since $e \leqslant b \leqslant r$, this forces the desired conclusion $e=b$.

Lemma 8.14. For some $y$ in $N-W$, we have $B^{y} \cap A_{1}=1$. 
Proof. If false, then by the preceding lemma all $N$-conjugates of $B$ other than $B$ itself lie in $A_{1}$. Since $W_{1}=W \cap K$ acts transitively on $A_{1}^{\#}$ and trivially on $B$, it follows now that $A^{\#}$ has precisely two conjugacy classes under the action of $N$ : namely, $A_{1}^{\#} \cup B^{\#}$ and $A-\left(A_{1} \cup B\right)$.

We consider the square of the conjugacy class of $A_{1}^{\#} \cup B^{\#}$ in the group ring $Z[A]$. We obtain, with $b=m(B)$ once again,

$$
\left(2^{r}+2^{b}-2\right) 1+\left(2^{r}-2\right) A_{1}^{\#}+\left(2^{b}-2\right) B^{\#}+1\left(A-\left(A_{1} \cup B\right)\right)
$$

where the coefficients represent the number of times the given set appears in the enumeration. Since we must end up with a linear combination of conjugacy classes of $A$ under the action of $N$, the only possibility is that $b=r$.

We conclude now from Lemma 8.12 that $A_{1}$ is the only $N$-conjugate of $B$ distinct from $B$, which implies that $|N: W|=2$.

Let $S$ be a Sylow 2-subgroup of $N$. Then $A \subseteq S$ as $A \triangleleft N$ and $S \in$ $S(G)$ as $A \in N(G)$. By the preceding equality, there exists $y$ in $N$ which interchanges $B$ and $A_{1}$ under conjugation. Since $y$ centralizes $Z(S) \subseteq A$, it follows that $Z(S) \cap A_{1}=Z(S) \cap B=1$. We can clearly choose $S$ so that $S \cap K \in S(K)$. If $S \cap K$ is nonabelian, then $A_{1}=(S \cap K)^{\prime} \subseteq Z(S)$, which is not the case. Hence $S \cap K=A_{1}$ and $K \cong L_{2}\left(2^{r}\right)$. Likewise no element of $W \cap S$ induces a field automorphism of $K$ of order 2, otherwise $A_{1} \cap S^{\prime} \subseteq$ $A_{1} \cap Z(S) \neq 1$. Hence $W \cap S=A_{1} C_{S}(K)$. But by definition of $A$ and $\bar{B}$, clearly $B \in M\left(C_{S}(K)\right)$. Hence if $C_{S}(K) \supset B$, it would follow that $B \cap$ $\mho^{1}\left(C_{S}(K)\right) \neq 1$. However, this is impossible as $\mho^{1}(S) \subseteq Z(S)$ and $B \cap Z(S)$ $=1$. Thus $C_{S}(K)=B$ and we conclude that $W \cap S=A_{1} B=A$.

Hence $S=\left(A_{1} \times B\right)\langle y\rangle$ with $y^{2} \in A=A_{1} B$ and $y$ interchanging $A_{1}$ and $B$. One easily proves in this situation by induction on the order $2^{r}$ of $B$ that $y$ can be taken to be an involution. Indeed, if we take $b$ in $B^{\#}$ and apply induction to $S /\left\langle b, b^{y}\right\rangle$, we see that we can choose $y$ so that $y^{2} \in$ $\left\langle b, b^{y}\right\rangle$. Then $\langle b, y\rangle \cong D_{8}$, and so $\langle b, y\rangle-\left\langle b, b^{y}\right\rangle$ contains an involution which we can take as $y$. Thus $S \cong E_{2^{r}} \sim E_{2}$, and now (2.74) yields a contradiction.

Now we can prove

LEMMA 8.15. One of the following holds:

(i) $A_{1} \triangleleft N$; or

(ii) $N$ acts transitively on $A^{\#}$.

Proof. Since $W \cap K$ acts transitively on $A_{1}^{\#}$ and trivially on $B$, conjugation by $W \cap K$ divides $A-B$ into classes of size $2^{r}-1$. Conjugating $B^{\#}$ by the element $y$ of Lemma 8.14 adds one element to each of these classes with the exception of the class $A_{1}^{\#}$. Hence the $N$-classes of $A-A_{1}$ are unions of 
sets each of size $2^{r}$ and each containing an element of $B^{\#}$.

Let $C_{1}$ be the $N$-conjugacy class of $A_{1}^{\#}$. To prove the lemma, we must show that either $C_{1}=A_{1}^{\#}$ or $A^{\#}$, so suppose false, in which case $C_{1}$ contains an element $b_{1}$ of $B^{\#}$ and $A^{\#}$ has an $N$-conjugacy class $C_{2}$ distinct from $C_{1}$. Likewise $C_{2}$ contains an element $b_{2}$ of $B^{\#}$. Furthermore, as $\left|A_{1}^{\#}\right|=2^{r}-1$, it follows from the preceding paragraph that

$$
\left|C_{1}\right| \equiv-1\left(\bmod 2^{r}\right) \text { and }\left|C_{2}\right| \equiv 0\left(\bmod 2^{r}\right) \text {. }
$$

On the other hand, by Lemma 8.8 , we have $C_{N}\left(b_{i}\right) \subseteq W, i=1,2$. Hence for $i=1$ and 2, we have

$$
\left|C_{i}\right|=\left|N: C_{N}\left(b_{i}\right)\right|=|N: W|\left|W: C_{W}\left(b_{i}\right)\right| .
$$

But now using our congruence for $\left|C_{1}\right|$, it follows that $|N: W|$ is odd. Hence by our congruence for $\left|C_{2}\right|$, we conclude that $\left|W: C_{W}\left(b_{2}\right)\right| \equiv 0\left(\bmod 2^{r}\right)$.

We shall now contradict this last conclusion. We know that $W$ normalizes $B$ and consequently $\left|W: C_{W}\left(b_{2}\right)\right| \leqslant\left|B^{\#}\right|$. But $\left|B^{\#}\right| \leqslant\left|A_{1}^{\#}\right|<2^{r}$, so $\left|W: C_{W}\left(b_{2}\right)\right|<2^{r}$, giving the desired contradiction.

As a corollary of our argument, we have

Lemma 8.16. If $A_{1} \triangleleft N$, then the $N$-conjugacy classes of $A-A_{1}$ each have size divisible by $2^{r}$ and are disjoint from $A_{1}$.

We shall next eliminate the second possibility of Lemma 8.15 and thus prove LEMma 8.17. We have $A_{1} \triangleleft N$.

Proof. Suppose false in which case $N$ acts transitively on $A^{\#}$. By Lemma $8.12 C_{N}(b) \subseteq W$ for $b \in B^{\#}$, whence $|N: W|$ is odd. Since $A \in N$, we can thus choose $S \in S$ with $S \subseteq W$. Let $T=C_{S}\left(A_{1}\right)$. Because $S$ acts on $K$, $|S: T| \leqslant 2$. As $S$ normalizes $B, A_{1} \subset C_{A}(T)$ and we have that $T$ centralizes an element of $\left(B^{y}\right)^{\#}$ for any $y$ in $N$. By Lemma 8.12, $T \subseteq W^{y}$. By Lemma 8.14 pick $y$ so that $B^{y} \cap A_{1}=1=B^{y} \cap B$. as $T$ centralizes $A_{1}$, it follows that $T$ centralizes $B$ and $T=C_{S}(A)$. Clearly $A=\Omega_{1}(T)$, so by Proposition $3.1|S: T|=2$ and $S-T$ contains an involution $t$. As $G$ is simple, $t$ is conjugate in $H=N_{G}(D)$ to $a$ in $A$ where $D \subseteq S$ is chosen as in (2.70). We know that $t$ acts on $K \cong L_{2}\left(2^{r}\right)$ as a field automorphism, whence $C_{S}(t)$ has rank at most $1+r / 2+b$, where $b=m(B)$. It follows from $r \geqslant 3$ that $r \geqslant 4$ and the fusion cannot occur in $N_{G}(S)$. Since $H=H^{*} N_{H}(S)$, the fusion occurs in $H^{*}$. By (2.65(iii)), at $\in Z(D)$, whence $D \cap A \subseteq C_{A}(t)$ and the image $\bar{A}$ in $\bar{H}=H / D$ has rank $\geqslant r / 2 \geqslant 2$. For $b \in A-A \cap D$, $\left|D: C_{D}(b)\right| \leqslant|S: T|=2$. Thus for any $h \in H,\left\langle\bar{b}, \bar{b}^{\bar{h}\rangle}\right\rangle$ is a dihedral group centralizing a subgroup of index at most 4 in $D$. Thus the core of this dihedral 
group has order at most 3, contradicting the structure of $\bar{H}$ given in (2.70).

We can now give a fairly complete description of a Sylow 2-subgroup of $G$.

Pick $b$ in $B^{\#}$ so that a Sylow 2-subgroup $P$ of $C_{G}(b)$ has maximal possible order. By Lemma 8.8, $P$ normalizes $K$ and so $T=P \cap K \in S(K)$. Without loss we can assume $A \subseteq P$ and we let $S$ be a Sylow 2-subgroup of $G$ containing $P$. Then $A \triangleleft S$ as $A \in N(S)$ and so $S \subseteq N$. Furthermore, $Z=Z(S) \subseteq A$ for the same reason. By Lemma 8.16, the number of $N$-conjugates of every element of $A-A_{1}$ is a multiple of $2^{r}$. In particular, this number is even and so no element of $A-A_{1}$ lies in $Z$, whence $Z \subseteq A_{1}$. Thus $|Z| \leqslant 2^{r}$. On the other hand, $[S, b] \subseteq Z$ as $S$ has class 2 . But $|[S, b]|=\left|S: C_{S}(b)\right|=|S: P|$ and so is a multiple of $2^{r}$. Hence $|Z|=2^{r}$ and so $Z=A_{1}$. This in turn implies that no element of $P$ induces a nontrivial outer automorphism of $K$, as otherwise $A_{1}$ would not lie in $Z(P)$. It follows therefore that $P=T \times C_{P}(K)$. Since $B \cap$ $Z=1$ and $B \in M\left(C_{P}(K)\right.$ ), we also have that $B=C_{P}(K)$ (using the fact that $S^{\prime}$ and $\mho^{1}(S)$ lie in $Z$ ). Thus $P=T \times B$. In addition, $P \triangleleft S$ and as $Z \subseteq$ $P$, we must have $S / P$ elementary, again as $S^{\prime}$ and $\mho^{1}(S)$ lie in $Z$. Our conditions also force $|S / P|=2^{r}$. Since each element of $A-A_{1}$ has a multiple of $2^{r} N$-conjugates, it follows now that $C_{S}(x)=P$ for $x$ in $A-A_{1}$. Since each such $x$ is conjugate to an element of $B^{\#}$, we conclude from our maximal choice of $b$ that each element of $A-A_{1}$ is extremal in $S$.

We summarize these conclusions:

Lemma 8.18. There exists a Sylow 2-subgroup $S$ of $G$ with the following properties:

(i) $A \subseteq S \subseteq N$ and $T=S \cap K \in S(K)$;

(ii) $A_{1}=Z(S)=Z(T)=[S, x]$ for any $x$ in $A-A_{1}$;

(iii) Every $x$ in $A-A_{1}$ is extremal in $S$ and $P=C_{S}(x)=T \times B$;

(iv) $P \triangleleft S$ and $S / P$ is elementary of rank $r$.

We fix such a Sylow 2-subgroup $S$ of $G$ and we next prove

Lemma 8.19. We have $K \cong L_{2}\left(2^{r}\right)$ and $P=A$.

Proof. Suppose false, in which case $K \cong S z\left(2^{r}\right)$ or $U_{3}\left(2^{r}\right)$. By Proposition 3.1, $A_{1}$ is not strongly closed in $S$. Hence some $z$ in $A_{1}^{\#}$ is conjugate in $G$ to $y$ in $S-A_{1}$. By the preceding lemma (or Lemma 8.16), no involution of $A-A_{1}$ is central and so $y \in S-P$ (as $\Omega_{1}(P)=A$ ). As usual, we may assume that this conjugacy occurs in $M=N_{G}(D)$ for some $D$ in the Goldschmidt conjugation family $D$. Since $N_{M}(S)$ normalizes $A_{1}=Z(S)$ and $M=M^{*} N_{M}(S)$, where $M^{*}=O^{2^{\prime}}(M)$, we can suppose that this conjugacy takes place in $M^{*}$.

Since $z \in A_{1} \subseteq Z=\Omega_{1}(Z(D))$, also $y \in Z$. Thus $D \subseteq C_{S}(y)$ and as $y \notin P$, Lemma 8.18(iii) implies that $D \cap A=A_{1}$. It follows that 
$D \cap P$ is isomorphic to a subgroup of $T$ containing $A_{1}$. Clearly $D \subset S$. If $|S: D|=2$, then necessarily $|B|=2$ and $D \cap P$ must project onto $P / B \cong T$, whence $A_{1}=(D \cap P)^{\prime}$. But this is impossible as $M^{*}$ centralizes $D^{\prime}$ by (2.65(iii)) and $M^{*}$ does not centralize $z \in A_{1}$. Hence $|S: D| \geqslant 4$ and so if we set $\bar{M}=M / D C_{M}(D)=M / D O(M)$, we have that $\bar{M}^{*} / O(\bar{M}) \cong L_{2}\left(2^{m}\right)$ for some $m \geqslant 2$.

We argue next that $\bar{M}^{*} \cong L_{2}\left(2^{m}\right)$. To prove this, we need only show that $\bar{S}$ centralizes $O\left(\bar{M}^{*}\right)$. Indeed, if that is the case, then $C_{\bar{M}^{*}}\left(O\left(\bar{M}^{*}\right)\right)$ covers $\bar{M}^{*} / O\left(\bar{M}^{*}\right) \cong L_{2}\left(2^{m}\right)$. However, as the latter groups have no nontrivial perfect central extensions by a group of odd order, it follows that $\bar{M}^{*}=\bar{L} \times O\left(\bar{M}^{*}\right)$, where $\bar{L} \cong L_{2}\left(2^{m}\right)$. But then $O^{2^{\prime}}\left(\bar{M}^{*}\right)=\bar{L}$. Since $\bar{M}^{*}=O^{2^{\prime}}(\bar{M}), O^{2^{\prime}}\left(\bar{M}^{*}\right)=$ $\bar{M}^{*}$ and we conclude that $\bar{M}^{*}=\bar{L} \cong L_{2}\left(2^{m}\right)$, as claimed.

Since $D \cap A=A_{1}, \bar{B} \neq 1$. But $C_{S}(b)=P$ for $b$ in $B^{\#}$ and consequently $C_{Z}(\bar{b})=A_{1}=Z(S)$ for $\bar{b}$ in $\bar{B}^{\#}$. However, by the structure of $L_{2}\left(2^{m}\right), N_{\bar{M}^{*}}(\bar{S})$ acts transitively on $\bar{S}^{\#}$ and consequently $C_{Z}(\bar{u})=Z(S)$ for all $\bar{u}$ in $\bar{S}^{\#}$. But now if $\bar{S}$ does not centralize $O(\bar{M})$, then $\bar{S}$ does not centralize $\bar{F}=\left[C_{O\left(\bar{M}^{*}\right)}(\bar{u}), \bar{S}\right]$ for some $\bar{u}$ in $\bar{S}^{\#}$ as $S \cong E_{2}, m \geqslant 2$. However, $\bar{F} \subseteq O^{2}\left(\bar{M}^{*}\right)$ and so $\bar{F}$ acts faithfully on $Z$ by (2.65(iv)). Hence by Thompson's $A \times B$-lemma, $\bar{F}$ must act faithfully on $C_{Z}(\bar{u})=Z(S)$. But $\bar{S}$ centralizes $Z(S)$ and $\bar{F}=[\bar{F}, \bar{S}]$, so $\bar{F}$ must centralize $Z(S)$, whence $\bar{F}=1$, which is not the case. This contradiction establishes that $\bar{M}^{*}$ has the asserted structure.

Set $E=\mho^{1}(D)$, so that $E \subseteq Z(S)=A_{1}$. Since $D \cap P$ is isomorphic to a subgroup of $T$ containing $A_{1}$, it is straightforward to show by counting the number of square roots of elements of $E$ in $D \cap P$ that

$$
\begin{array}{ll}
\left|D \cap P: A_{1}\right| \leqslant|E| & \text { if } K=S z\left(2^{r}\right), \\
\left|D \cap P: A_{1}\right| \leqslant|E|\left|A_{1}\right| & \text { if } K=U_{3}\left(2^{r}\right) .
\end{array}
$$

Since correspondingly $|T|=\left|A_{1}\right|^{2}$ or $\left|A_{1}\right|^{3}$, we conclude in both cases that

$$
|D \cap P|\left|A_{1}\right| \leqslant|T||E| .
$$

We shall now establish a second inequality for $|D \cap P|\left|A_{1}\right|$. We use (2.66(iv)) which is applicable as $\bar{M}^{*} \cong L_{2}\left(2^{m}\right)$. It yields

$$
|S: D| \leqslant\left|A_{1}: E\right|
$$




$$
|P: D \cap P|=|P D: D| \leqslant|S: D| \leqslant\left|A_{1}: E\right| .
$$

Since $|S: P|=\left|A_{1}\right|$ by the preceding lemma, this implies

$$
|S: D \cap P|=|S: P||P: D \cap P| \leqslant\left|A_{1}\right|\left|A_{1}: E\right|,
$$

which in turn yields

$$
|D \cap P|\left|A_{1}\right| \geqslant\left|S: A_{1}\right||E| .
$$

Comparison with our first estimate gives $|T| \geqslant\left|S: A_{1}\right|=|P|$. However, this is impossible as $P=T \times B$ and $B \neq 1$. This establishes the lemma.

In treating the remaining case, we first prove

LEMMA 8.20. The following conditions hold:

(i) $S \triangleleft N$;

(ii) $N=S W$;

(iii) $S \cap W=A=P$.

Proof. We know from Lemma 8.18 that $C_{S}(B)=P$. We claim first that $N_{S}(B)=P$. If not, then $b^{y} \in B$ for some $b$ in $B^{\#}$ and some $y$ in $S-P$. But then $1 \neq[b, y] \in B \cap S^{\prime} \subseteq B \cap Z(S)$. However, $B \cap Z(S)=1$ by Lemma 8.18 , so our assertion holds.

We know that the $N$-conjugates of $B^{\#}$ are disjoint and as $N_{S}(B)=P$ with $|S: P|=\left|A_{1}\right|$, it follows that the $S$-conjugates of $B^{\#}$ include all $N$-conjugates of $B^{\#}$. Hence $N=S N_{N}(B)=S W$ by Lemma 8.11(i). Since $S \cap W=P$, it remains only to show that $S \triangleleft N$.

We argue first that $N$ is 2-constrained with $O(N)=1$. Indeed, $O(N)$ centralizes $A$ and so for $b$ in $B^{\#}, O(N) \subseteq C_{G}(b)$ and $A=P \in S\left(C_{G}(b)\right)$ by Lemmas 8.18 and 8.19. Since $A$ is elementary, every component of $L_{b}$ is isomorphic to $L_{2}\left(2^{m}\right)$ for some $m$. Since $O(N)$ centralizes $A \cap L_{b} \in$ $S\left(L_{b}\right), O(N)$ centralizes $L_{b}$ by $(2.20($ iii)). Furthermore, $O(N)$ centralizes $O_{2}\left(C_{G}(b)\right) \subseteq A$. Since $O\left(C_{G}(b)\right)=1$, we conclude now from (2.8) that $O(N)$ $=1$. Furthermore, $L(N)$ centralizes $A$ as $L(N)$ and $A$ are each normal in $N$. But $S \cap L(N) \in S(L(N))$ and $C_{S}(A) \subseteq C_{S}(B)=A$. Hence $A \cap L(N)$ is a Sylow 2-subgroup of $L(N)$ and lies in $Z(L(N))$, which is clearly possible only if $L(N)=1$. We conclude now from (2.9) that $N$ is 2-constrained, which proves our assertions.

Finally set $Q=O_{2}(N)$, so that $A \subseteq Q \subseteq S$. Since $A_{1}=Z(S), S$ centralizes both $A_{1}$ and $Q / A_{1}$ and so stabilizes the chain $Q \supset A_{1} \supset 1$. But $A_{1}$ $\triangleleft N$ by Lemma 8.17 and $C_{N}(Q) \subseteq Q$ as $N$ is 2-constrained with $O(N)=1$. Hence the stabilizer of this chain is precisely $O_{2}(N)=Q$ and we conclude that $S=Q \triangleleft N$. 
We can now pin down $S$ precisely.

LEMMA 8.21. The following conditions hold for some subgroup $R$ of $S$ :

(i) $S=R B$ with $R \cap B=1, A_{1} \subseteq R$, and $R$ is invariant under $W$.

(ii) $|R|=2^{2 r}$ and $R$ is either elementary or homocyclic abelian.

Proof. We have that $W$ acts on $S$ and so $W$ acts on $\bar{S}=S / A_{1}$. Since $A_{1} \supseteq \mho^{1}(S), \bar{S}$ is elementary abelian. Since $\bar{B}$ is $W$-invariant, $\bar{S}=\bar{B} \times \bar{R}$ for some $W$-invariant subgroup $\bar{R}$ of $\bar{S}$. Thus the inverse image $R$ of $\bar{R}$ in $S$ is $W$-invariant, contains $A_{1}$, and is disjoint from $B$. Since also $S=R B, R$ satisfies the conditions of (i). Moreover, $|R|=2^{2 r}$ as $|S: A|=2^{r}$.

Suppose $R$ is not elementary. For $b$ in $B^{\#},[S, b]=[R, b]=A_{1}$ as $C_{S}(b)=A$. Since $W$ acts transitively on $A_{1}^{\#}$ and trivially on $b$, it follows that $W$ also acts transitively on $\bar{R}$. Hence if some coset of $A_{1}$ in $R$ distinct from $A_{1}$ contains an involution, all such cosets do and as $A_{1} \subseteq Z(R)$, it would follow at once that $R$ had exponent 2 and was elementary, which is not the case. Thus $A_{1}=\Omega_{1}(R)$. Since $A$ is not strongly closed in $S, S-A$ contains an involution $y$ and $y$ has the form $x b$, where $x \in R-A_{1}$ and $b \in B^{\#}$. Then $b$ must invert $x$ and as $W$ centralizes $b$ and acts transitively on $\bar{R}$, this implies that $b$ inverts $R$, forcing $R$ to be abelian. The only possibility is that $R$ is homocyclic and we conclude that (ii) holds.

We next prove

LEMMA 8.22. $R$ is elementary abelian.

Proof. Suppose false, in which case $R$ is homocyclic abelian and $\Omega_{1}(R)$ $=A_{1}$. Since $A_{1}$ is not strongly closed in $S$, it follows, as usual, that $z \sim y$ in $M=N_{G}(D)$ for some $z$ in $A_{1}^{\#}, y$ in $S-A$, and $D$ in $D$, and, moreover, that this conjugacy can be assumed to occur in $M^{*}=O^{2^{\prime}}(M)$. We have $y=$ $x b$ for some $x$ in $R-A_{1}$ and $b$ in $B^{\#}$. Furthermore, $z \in Z=\Omega_{1}(Z(D))$, so $y \in Z$ and hence $D \subseteq C_{S}(y)$ and $D \cap A=A_{1}$.

Observe next that $b$ inverts $R$ as we have shown in the preceding lemma and consequently $C_{R}(y)=A_{1}$. Thus $R \cap D=A_{1}$ and so if we set $\bar{M}=$ $M / D C_{M}(D)$, we have that $|\bar{S}| \geqslant\left|R / A_{1}\right|=2^{r}$, whence $|\bar{S}|>2$. Since $D \cap A=$ $A_{1}, \bar{B} \neq 1$ and we conclude now exactly as in Lemma 8.19 that $\bar{M}^{*} \cong L_{2}\left(2^{m}\right)$ for some $m$. Our conditions imply that $m \geqslant r$. On the other hand, if $b_{1} \in$ $B^{\#}$ with $b_{1} \neq b$, we claim that $b_{1}$ does not invert any element of $R-A_{1}$. Indeed, if $b_{1}$ inverted such an element $u$, then $u \in C_{S}\left(b b_{1}\right)$, contrary to the fact that $C_{S}\left(b b_{1}\right)=A$. This means that $x_{1} b_{1}$ is not an involution for any $x_{1}$ in $R-A_{1}$ and any $b_{1} \neq b$ in $B^{\#}$. From this we conclude that $Z=A_{1} \times$ $\langle y\rangle$ and so $m(Z)=r+1$. However, as $|\bar{S}|=2^{m}$ with $m \geqslant r \geqslant 2$ and $\bar{M}^{*}$ $\cong L_{2}\left(2^{m}\right)$, we see that $(2.66(\mathrm{iv}))$ is contradicted. 
We are left finally with the case that $R$ is elementary. This time we use the fact that $R$ is not strongly closed in $S$. Hence $z \sim y$ in $G$ for some $z$ in $R^{\#}$ and some $y$ in $S-R$. But as $C_{S}(b)=A$ for $b$ in $B^{\#}$, it is immediate that every involution of $S$ lies in $R$ or $A$. In particular, $y \in A-A_{1}$. We have shown above that no element of $A-A_{1}$ is conjugate to an element of $A_{1}$ and so also $z \in R-$ $A_{1}$. As usual, we can assume that this conjugation occurs in $M=N_{G}(D)$ for some $D$ in $D$. But $Z(D) \subseteq C_{S}(y) \cap C_{S}(z)=A \cap R=A_{1}$. Thus $M^{*}=O^{2^{\prime}}(M)$ centralizes $Z(D)$. Since $M^{*}$ centralizes $D / Z(D)$ by $\left(2.65\left(\right.\right.$ iii)), it follows that $O^{2}\left(M^{*}\right)$ centralizes $D$. Since $M=O^{2}\left(M^{*}\right) N_{M}(S)$ by the Frattini argument, we conclude that our conjugation takes place in $N_{M}(S)$. However, $R$ and $A$ are clearly the only two elements of $N(S)$ and consequently each must be normal in $N_{G}(S)$, whence $z$ and $y$ cannot be conjugate in $N_{M}(S)$. We have therefore proved

\section{LEMMA 8.23. Case 1 does not occur.}

It remains to treat Case 2. Thus $K \cong L_{3}\left(2^{n}\right), P S p\left(4,2^{n}\right)$, or $K / Z(K) \cong L_{3}(4)$. We put $n=2$ in the last case. We set $\bar{N}=N / C_{G}(A)$ and $W_{1}=W \cap K$. By (2.21(iii)), $\bar{W}_{1}=Z\left(\bar{W}_{1}\right) \times \bar{J}$, where $Z\left(\bar{W}_{1}\right)$ is cyclic of order dividing $2^{n}-1$ and $\bar{J} \cong S L\left(2,2^{n}\right) \cong L_{2}\left(2^{n}\right)$ is simple as $n \geqslant 2$. Again we choose $S$ in $S(G)$ to contain $A$ as well as a Sylow 2-subgroup $T$ of $K$. Again $A \in N(S)$ is normal in $S$ and so $S \subseteq N$.

The elimination of this case hinges on the following result:

LEMMA 8.24. $\bar{J}$ is a component of $L(\bar{N})$.

Proof. Since $A \in N(G)$, we have $Z(S) \subseteq A$ and so $S^{\prime} \subseteq A$. Thus $\bar{S}$ is abelian, and it follows now from the structure of groups with abelian Sylow 2-subgroups that $\bar{N} / L_{2} \cdot(\bar{N})$ is solvable. As $\bar{J}$ is perfect, we conclude that $\bar{J} \subseteq L_{2}{ }^{\prime}(\bar{N})$. By (2.21(vii)) $C_{A_{1}}(\bar{t})=T^{\prime} \subseteq Z(S)$ for any $\bar{t}$ in $\bar{T}^{\#}$. Since $K$ centralizes $B$, we have $C_{A}(\bar{t})=T^{\prime} B=C_{A}(T)$. We claim that $T$ centralizes $\bar{F}=O\left(L_{2}(\bar{N})\right)$. Indeed $C_{\bar{F}}(\bar{t})$ normalizes $C_{A}(\bar{t})=C_{A}(\bar{T})$ whence $\bar{F}=\left\langle C_{\bar{F}}(\bar{t}) \mid \bar{t} \in \bar{T}^{\#}\right\rangle$ normalizes $C_{A}(\bar{T})$. Since $C_{A}(\bar{T})=T^{\prime} \times B$, we see that $\bar{T}$ centralizes $A / C_{A}(\bar{T})$ and $C_{A}(\bar{T})$, and hence so does $[\bar{F}, \bar{T}]$. We conclude that $[\bar{F}, \bar{T}]$ centralizes $A$ and $[\bar{F}, \bar{T}]$ $=1$. Consequently $[\bar{F}, \bar{J}]=1$, and $\bar{J} \subseteq L(\bar{N})$ by $(2.10)$.

We must show that $\bar{J}$ is a component of $L(\bar{N})$. By $(2.65(\mathrm{v}))$ if $\bar{N}_{1}$ and $\bar{N}_{2}$ are distinct components of $L(\bar{N})$, then in an $L(\bar{N})$-composition series for $A, \bar{N}_{1}$ and $\bar{N}_{2}$ act nontrivially on distinct composition factors $V_{1}$ and $V_{2} . \bar{J}$ centralizes $B$ and has just one nontrivial composition factor on $A_{1}$ by (2.21). Thus $\bar{J}$ cannot act nontrivially on both $V_{1}$ and $V_{2}$. Consequently $\bar{J}$ cannot project nontrivially on both $\bar{N}_{1}$ and $\bar{N}_{2}$, and we may assume $\bar{J} \subseteq \bar{N}_{1}$.

We claim $\bar{N}_{1}$ is of type $L_{2}\left(2^{m}\right)$. If not, then by (2.65), $\bar{N}_{1}$ is of type $L_{2}(q)$, $q \equiv 3,5 \bmod 8, q>5, J_{1}$ or Ree type. $\bar{T}$ is of rank at least 2 , and by the argument 
given above for $\bar{F}, \bar{T}$ centralizes any $\bar{T}$-invariant subgroup of odd order of $\bar{N}_{1}$. But if $\bar{N}_{1}$ is of type $L_{2}(q)$, then we know from the subgroup structure of $L_{2}(q)$ that the centralizer of $\bar{t}$ in $\bar{N}_{1} / Z\left(\bar{N}_{1}\right)$ is dihedral of order $q+1$ or $q-1$ according as $q \equiv 3 \bmod 8$ or $q \equiv 5 \bmod 8$. In either case $\bar{T}$ does not centralize $O\left(C_{\bar{N}_{1}}(\bar{t})\right)$. Thus $\bar{N}_{1}$ is not of type $L_{2}(q)$ with the given restrictions on $q$. Likewise if $\bar{N}_{1}$ were of Ree type, then by (2.26(v)) the same argument would apply to the centralizer in $\bar{N}_{1}$ of a subgroup of rank 2 of $\bar{T}_{1}$. We are left with the possibility that $\bar{N}_{1}$ is of type $J_{1}$. In this case we know [23, Section 1] that all involutions of $N_{1}$ are conjugate and that for $\bar{t} \in \bar{T}^{\#}, \bar{t}$ has a conjugate $\bar{u}$ such that $\langle\bar{u}, \bar{t}\rangle$ is dihedral of order 38. Hence $O(\langle\bar{u}, \bar{t}\rangle)$ has order 19 and acts faithfully on $A /\left(C_{A}(\bar{t}) \cap C_{A}(\bar{u})\right)$ which has rank at most $2 m\left(A / C_{A}(\bar{t})\right)$. But $\bar{T} \subseteq \bar{N}_{1}$ implies $m(\bar{T}) \leqslant m\left(J_{1}\right)=3$ whence $m\left(A / C_{A}(\bar{t})\right)=m\left(A_{1} / C_{A_{1}}(\bar{t})\right) \leqslant 6$ by $(2.21(i i))$. Thus $\left.O(\bar{u}, \bar{t}\rangle\right)$ acts faithfully on an elementary abelian group of order at most $2^{6}$ which is impossible.

Finally we have that $\bar{N}_{1}$ is of type $L_{2}\left(2^{m}\right)$. We know by (2.21(ii)) that $\left(A: C_{A}(\bar{t})\right)=2^{n}$ where $\bar{J} \cong L_{2}\left(2^{n}\right)$. Hence if $V$ is any nontrivial $\bar{N}_{1}$-composition factor of $A$, we have $\left(V: C_{V}(\bar{t})\right) \leqslant 2^{n}$ whence $m \leqslant n$ by (2.66(ii)). We conclude $\bar{J}=\bar{N}_{1}$.

We now strengthen the last conclusion.

\section{LEMMA 8.25. $\bar{J}$ is normal in $\bar{N}$.}

PRoof. If $K / Z(K) \cong L_{3}\left(2^{n}\right)$, then $r=m(K / Z(K))=2 n$. Since $m(G) \leqslant 2 r$, it follows that $m(A) \leqslant 4 n$. But now (2.66) implies that $L(\bar{N})$ has at most 2 components isomorphic to $L_{2}\left(2^{n}\right)$. Since $\bar{J}$ is a component of $L(\bar{N}), O^{2}(\bar{N})$ must then leave $\bar{J}$ invariant. Since $\bar{S}$ is abelian, it also normalizes $\bar{J}$ and we conclude that $\bar{J} \triangleleft \bar{N}$.

Suppose then that $K \cong P S p\left(4,2^{n}\right)$, whence $r=m(K)=3 n$ by (2.21(i)). In this case, $m(A) \leqslant 6 n$ and so $L(\bar{N})$ has at most 3 components isomorphic to $L_{2}\left(2^{n}\right)$. If the number is less than $3, \bar{J} \triangleleft \bar{N}$ as in the preceding case. We can therefore assume that $L(\bar{N})$ has 3 isomorphic components $\bar{J}=\bar{J}_{1}, \bar{J}_{2}, \bar{J}_{3}$. In this case $m(A)=6 n$ and $\bar{J}=\bar{J}_{1}$ acts faithfully and irreducibly on $A_{0}=$ $\left[A, \bar{J}_{1}\right]$ which is of rank $2 n$, by $(2.65(\mathrm{v}))$. Since $\bar{J}$ centralizes $A / A_{1}$, it follows that $A_{0} \subseteq A_{1}$. But by (2.21(v)), $A_{1}$ has no faithful irreducible $\bar{J}$-submodules.

Now we can eliminate Case 2 and thus attain our objective:

Lemma 8.26. $N_{G}(A)$ normalizes $K$ for some choice of $A$.

Proof. Since $\bar{N}$ normalizes $\bar{J}$, it leaves $C_{A}(\bar{J})$ invariant. If $K / Z(K) \cong$ $L_{3}\left(2^{n}\right)$, then $\bar{J}$ acts faithfully and irreducibly on $A_{1} / Z(K)$ and $Z(K) \subseteq B$, so $C_{A}(\bar{J})=B$. Thus $\bar{N}$ leaves $\bar{B}$ invariant and so $B \triangleleft N$. But then $N=W$ by Lemma 8.11(i), contrary to our assumption $W \subset N$. Hence $K \cong P S p\left(4,2^{n}\right)$.

In this case $A_{0}=C_{A_{1}}(\bar{J})$ has rank $n$ by $(2.21(\mathrm{v}))$ and we have $C_{A}(\bar{J})=$ 
$A_{0} B$. Our argument yields that $A_{0} B$ is normal in $N$. Furthermore, $Z\left(\bar{W}_{1}\right)$ acts transitively and regularly on $A_{0}^{\#}$ by $(2.21(\mathrm{v}))$ and trivially on $B$. Thus the situation is very analogous to that which we had in Case 1 . We know that any two $N$-conjugates of $B$ intersect trivially by Lemma 8.12. Likewise $N$ does not normalize $B$ as $W \subset N$. We conclude now as in Case 1 that the $N$-classes of $A_{0} B-B$ have size divisible by $\left|A_{0}\right|=2^{n}$ and intersect $B$ nontrivially. From this it follows, as before, that $2^{n}$ divides $\left|S: C_{S}(b)\right|$ whence $[S, b]=A_{0}$ for $b$ in $B^{\#}$, which in turn yields $C_{S}(b)=N_{S}(B)$ for $b$ in $B^{\#}$. As before, the $S$-conjugates of $B^{\#}$ fill up $A_{0} B-B$ and we conclude as in Lemma 8.20 that $N=S W$.

To obtain a final contradiction, we exploit the fact that $T=S \cap K$ possesses a second elementary subgroup $A_{1}^{*} \neq A_{1}$ of rank $3 n$ and that $N_{K}\left(A_{1}^{*}\right)$ has the same structure as $N_{K}\left(A_{1}\right)$. Then $A^{*}=A_{1}^{*} \times B$ satisfies the same criteria for selection as $A$ inasmuch as $m\left(A^{*}\right)=m(A), a \in B \subseteq A^{*}$ and $K$ is a component of $L_{a}$. This means that we can work with $A^{*}$ just as well as with $A$. Hence if $N^{*}=N_{G}\left(A^{*}\right)$ and $W^{*}=N_{N^{*}}(K)$, it follows from our assumption that also $W^{*} \subset N^{*}$. Therefore all the conclusions we obtained about $N$ hold as well for $N^{*}$. In particular, setting $\bar{N}^{*}=N^{*} / C_{G}\left(A^{*}\right)$ and defining $\bar{J}^{*}$ by analogy with $\bar{J}$, we conclude that $A_{0}^{*} B$ is normal in $N^{*}$, where $A_{0}^{*}=C_{A_{1}^{*}}^{*}\left(J^{*}\right)$. Since $S \subseteq N^{*}$, it follows that $S$ normalizes $A_{0}^{*} B$.

Our argument yields that $S$ normalizes $A_{0} B \cap A_{0}^{*} B$. But $A_{0}$ and $A_{0}^{*}$ lie in $Z(T)$ by $\left(2.21\right.$ (vii)), while $B \cap Z(T)=1$, so $A_{0} B \cap A_{0}^{*} B=\left(A_{0} \cap A_{0}^{*}\right) B$. However, $A_{0} \cap A_{0}^{*}$ is normalized by $\left\langle N_{K}\left(A_{1}\right), N_{K}\left(A_{1}^{*}\right)\right\rangle$, as is easily checked, and this group is $K$ by (2.36(iii)). Since $K$ is simple, this forces $A_{0} \cap A_{0}^{*}=$ 1 and we conclude that $S$ normalizes $B$. Thus $S \subseteq W=N_{N}(B)$ and so $N=$ $S W=W$, giving a final contradiction.

We assume henceforth that $A$ is chosen so that $N_{G}(A)$ normalizes $K$. As an immediate corollary, we have

\section{Lemma 8.27. We have $A \in M(G)$.}

Proof. We know that $N=N_{G}(A)$ contains a Sylow 2-subgroup $S$ of $G$. Since $S$ normalizes $K$, it normalizes $B=C_{A}(K)$. Since $B \neq 1$, it follows that $z \in B$ for some involution $z$ of $Z(S)$. Let $A^{*} \in M(S)$, so that $z \in$ $Z(S) \subseteq A^{*}$. By Lemma $8.2, K$ is a component of $L_{z}$ and so $K \in L\left(G ; A^{*}\right)$. Now our maximal choice of $A$ forces $m(A)=m\left(A^{*}\right)$ and so $A \in M(G)$.

By Lemma 8.5, $X_{A}$ does not normalize $K$. Hence for some $x$ in $A^{\#}$ and some component $J$ of $L_{x}$, we have that $J$ does not normalize $K$. We fix such an $x$ and $J$ and investigate the structure of $J$. Since $A \in M(G)$, it follows from (2.37) that $J$ is $A$-invariant. If $J \cong L_{2}(5)$, no involution of $C_{G}(x)$ 
induces a nontrivial outer automorphism of $J$ as $L_{2}(5)$ is evenly embedded in $L(G)$. Since either $J \cong L_{2}(5)$ or $J$ is of even type, it follows from (2.37) that $A \cap J \in M(J)$ in all cases. Furthermore, except possibly in the case $J \cong L_{2}(9)$, no element of $A^{\#}$ induces a nontrivial outer automorphism of $J$ by (2.37(iv)) and so except possibly in this case, we have $A=(A \cap J) C_{A}(J)$. Finally as $C_{G}(b)$ normalizes $K$ for $b$ in $B^{\#}$ by Lemma 8.8 , we have

$$
J \nsubseteq\left\langle C_{G}(b) \mid b \in B^{\#}\right\rangle .
$$

We shall use these facts in our analysis. We first prove

LEMMA 8.28. We have $J \not L_{2}(9)$.

Proof. Suppose $J \cong L_{2}(9)$. Set $A_{0}=A \cap J$, so that $A_{0} \cong E_{4}$. By (2.22), $N_{J}(A)=H \cong S_{4}$ and $A=A_{0} \times B_{0}$, where $B_{0}=C_{A}(H)$. Since $H$ normalizes $A$, it normalizes $K$. Hence $H$ normalizes both $A_{1}$ and $B$. Since $A_{0}$ is the unique subgroup of $A$ on which $H$ acts irreducibly and nontrivially, we have $A_{0} \subseteq A_{1}$ or $B$.

Suppose $A_{0} \subseteq A_{1}$, in which case $H$ acts faithfully on $K$ and $A_{1}=A_{0}$ $\times\left(A_{1} \cap B_{0}\right)$. Let $u$ be an involution of $H-H^{\prime}$. Then $u$ centralizes $B_{0}$ as well as a subgroup of index 2 in $A_{0}$ and consequently $\left|A_{1}: C_{A_{1}}(u)\right|=2$. But $K$ is of even type with $m(K / Z(K)) \geqslant 3$ and now (2.20) and (2.21) show that no involution of $N_{G}(K)$ can act on $A_{1}$ in this way. We conclude that $A_{0} \subseteq$ $B$. This in turn implies that $A_{1} \subseteq B_{0}$. Hence $u$ centralizes $A_{1}$. Since $u \notin$ $B_{0}, u \notin A_{1}$, and now by (2.20) and (2.21) some involution $v$ of $A_{1}\langle u\rangle-A_{1}$ centralizes $K$.

The group $T_{0}=A_{0}\langle v\rangle \cong D_{8}$ and centralizes $K$. Moreover, $A \subseteq T_{0} B_{0}=$ $T_{0} \times B_{0}$. Let $A_{0}^{*}$ be the four subgroup of $T_{0}$ other than $A_{0}$ and set $A^{*}=$ $A_{0}^{*} \times B_{0}$. Then $m\left(A^{*}\right)=m(A)$ and so $A^{*} \in M(G)$. Furthermore, $A_{0} \cap A_{0}^{*}$ $\neq 1$ and as $A_{0} \subseteq B$, it follows that $b \in A^{*}$ for some $b$ in $B^{\#}$. Since $K$ is a component of $\bar{L}_{b}$ by Lemma 8.2 , we have that $K \in L\left(G: A^{*}\right)$. Hence $A^{*}$ satisfies our initial conditions (a) and (b) as well as $A$ does. Since Lemmas 8.28.9 hold for an arbitrary clement of $N(G)$ satisfying these conditions, we conclude now if we set $B^{*}=C_{A^{*}}(K)$, that $C_{G}\left(b^{*}\right)$ normalizes $K$ for all $b^{*}$ in $\left(B^{*}\right)^{\#}$ (which is the analogue of Lemma 8.8). But $A_{0}^{*} \subseteq B^{*}$ as $A_{0}^{*} \subseteq T_{0}$ and $T_{0}$ centralizes $K$. Hence $C_{G}\left(a^{*}\right)$ normalizes $K$ for $\bar{a}^{*}$ in $\left(A_{0}^{*}\right)^{\#}$.

Since $I\left(T_{0}\right)=A_{0}^{\#} \cup\left(A_{0}^{*}\right)^{\#}$ and $A_{0} \subseteq B$, our argument yields that $\left.\left\langle C_{J}(t)\right| t \in I\left(T_{0}\right)\right)$ normalizes $K$. However, as $T_{0} \cong D_{8}$, it follows from (2.36(i)) that this group is $J$ itself. Thus $J$ normalizes $K$, contrary to our choice of $J$.

Since $J \nRightarrow L_{2}(9)$, we have that $A=(A \cap J) C_{A}(J)$. We put $A_{0}=A \cap J$ 
and $B_{0}=C_{A}(J)$. Thus $A=A_{0} B_{0}$ with $A_{0} \cap B_{0}=Z(J)$ and $A_{0} \in M(J)$. We also set $H=N_{J}\left(A_{0}\right)$. Our conditions imply that $B \cap B_{0}=1$.

We next prove

Lemma 8.29. We have $J \not L_{3}\left(2^{m}\right), \operatorname{Psp}\left(4,2^{m}\right), m \geqslant 2$, or a covering group of $L_{3}(4)$.

Proof. Suppose false and consider first the case that $Z(J) \neq 1$, whence $J / Z(J) \cong L_{3}(4)$ and $Z(J) \subseteq B_{0}$. We have that $H$ normalizes $A$ and so $H$ normalizes $K$, which implies that $H$ normalizes both $A_{1}$ and $B$. Set $\bar{A}=$ $A / Z(J)$. Then $H$ acts irreducibly on $\bar{A}_{0}$ by (2.21) and as $H$ acts trivially on $\bar{A} / \bar{A}_{0}$, it follows that $\bar{A}_{0} \subseteq \bar{A}_{1}$ or $\bar{B}$. In the latter case, $A_{0} \subseteq Z(J) B$. But $Z(J) \cap B=1$ as $Z(J) \subseteq B_{0}$ and $B \cap B_{0}=1$. Thus $A_{0}=Z(J) \times\left(A_{0} \cap B\right)$ with $A_{0} \cap B$ invariant under $H$. However, this is impossible as $H$ acts indecomposably on $A_{0}$, a fact which is easily checked using (2.16).

Hence we must have $\bar{A}_{0} \subseteq \bar{A}_{1}$, whence $A_{0} \subseteq Z(J) A_{1}$. The indecomposable action of $H$ on $A_{0}$ now forces $Z(J) \subseteq A_{1}$, so $A_{0} \subseteq A_{1}$. Since $B \cap$ $A_{1} \subseteq Z(K), m\left(B \cap A_{1}\right) \leqslant 2$, whence $m\left(B \cap A_{0}\right) \leqslant 2$. But $B \cap A_{0}$ is $H$-invariant and so $B \cap A_{0} \subseteq Z(J)$, whence $B \cap A_{0} \subseteq B \cap B_{0}=1$. But $B$ is $H$-invariant and $A_{0}$ covers the unique nontrivial irreducible factor $H$-module of $A$. Hence $H$ must act trivially on $B$ and we conclude that $B \subseteq B_{0}$, contrary to $B \cap B_{0}=1$.

Suppose next that $J \cong L_{3}\left(2^{m}\right)$ for some $m$, in which case $H$ acts irreducibly on $A_{0}$, which has rank $2 m \geqslant 4$. As above, we have that $H$ normalizes $K, A_{1}$, and $B$ and that $A_{0} \subseteq A_{1}$ or $B$. Moreover, if $A_{0} \subseteq A_{1}$, we reach the same contradiction as before, since we again must have $B \cap A_{0}=1$. Thus $A_{0}$ $\subseteq B$ and as $A=A_{0} B_{0}$ with $B \cap B_{0}=1$, we conclude that $A_{0}=B$.

Since $H$ centralizes $A / A_{0}$, it follows now that $H$ acts trivially on $A_{1}$. Since $H^{\prime}$ is perfect, $(2.20$ (iv)) and $(2.21(\mathrm{vi}))$ imply that $H$ centralizes $K$. Let $T_{0}$ be a Sylow 2-subgroup of $J$ containing $A_{0}$. Then $T_{0}$ contains a second elementary subgroup $B^{*}$ of the same rank as $B=A_{0}$ by (2.21) and if we set $A^{*}=A_{1} \times B^{*}$, it follows as in the preceding lemma that $A^{*}$ satisfies the same initial conditions as $A$ and that Lemma 8.8 holds as well for $A^{*}$ as it does for $A$. This means that $C_{G}(b)$ normalizes $K$ for all $b$ in $B^{\#} \cup\left(B^{*}\right)^{\#}$. By (2.21(i)) and (2.36(i)), $I\left(T_{0}\right)=B^{\#} \cup\left(B^{*}\right)^{\#}$ and $J=\left\langle C_{J}(t) \mid t \in I\left(T_{0}\right)\right\rangle$. Thus $J$ normalizes $K$, contrary to our choice of $J$.

Finally assume that $J=P \operatorname{SP}\left(4,2^{m}\right)$, in which case $H$ acts indecomposably on $A_{0}$ and $A_{0}$ possesses a single proper nontrivial $H$-submodule $C_{0}$. Then again $H$ normalizes $K, A_{1}$, and $B$ and considering the action of $H$ on $A / C_{0}$, it follows that $A_{0} \subseteq C_{0} A_{1}$ or $C_{0} B$. In the first case the indecomposable action 
of $H$ on $A_{0}$ together with the irreducible action of $H$ on $C_{0}$ force $C_{0} \subseteq$ $A_{1}$, whence $A_{0} \subseteq A_{1}$. If $B \cap A_{0}=1$, we reach the same contradiction as before. However, this time there is a second possibility: namely, $B \cap A_{0}=C_{0}=$ $Z(K) \cong E_{4}$, whence $m=2, K / Z(K) \cong L_{3}(4)$, and $A_{0}=A_{1}$. Passing to $\bar{A}$, we have that $\bar{B} \cap \bar{A}_{0}=1$ and now we conclude that $\bar{B} \subseteq \bar{B}_{0}$, whence $B \subseteq C_{0} B_{0}$. Since $B \cap B_{0}=1$, and $C_{0}$ is an irreducible $H$-module, this forces $B=C_{0}$ and so $A=A_{0}=A_{1}$, contrary to the fact that $A=A_{0} \times B_{0}$ with $B_{0} \neq 1$. Thus $A_{0} \subseteq C_{0} B$ and now the indecomposable action of $H$ on $A_{0}$ and $B \cap B_{0}=1$ force the same conclusion $A_{0}=B$ as before.

Now we can repeat the argument of the corresponding part of the preceding case. With $T_{0}, B^{*}, A^{*}$ having the corresponding meanings and using (2.20) and (2.21) we obtain that $C_{G}(b)$ normalizes $K$ for $b$ in $B^{\#} \cup\left(B^{*}\right)^{\#}$ and that $I\left(T_{0}\right)=B^{\#} \cup\left(B^{*}\right)^{\#}$. But now (2.36(i)) again implies that $J=\left\langle C_{J}(t)\right| t \in$ $\left.I\left(T_{0}\right)\right\rangle$, so $J$ normalizes $K$, again a contradiction.

Combining the last two lemmas we obtain as a corollary

LEMmA 8.30. We have $J \cong L_{2}\left(2^{m}\right), S z\left(2^{m}\right)$, or $U_{3}\left(2^{m}\right), m \geqslant 2$.

Thus it remains to treat the case in which $J$ is a "Bender" group. In this case $H$ is the semidirect product of a Sylow 2-subgroup $T_{0}$ of $J$ and a cyclic group $F$ of order $2^{m}-1$ if $J \cong L_{2}\left(2^{m}\right)$ or $S z\left(2^{m}\right)$ and of order dividing $2^{2 m}-1$ if $J \cong U_{3}\left(2^{m}\right)$, which acts transitively on $A_{0}^{\#}$. Again $H$ normalizes each of $K, A_{1}$, and $B$, so $A_{0} \subseteq A_{1}$ or $B$ and again the first inclusion leads to a contradiction. Thus $A_{0} \subseteq B$ and again our conditions force $A_{0}=B$. If $A=A_{1} \times$ $B$, then necessarily $A_{1} \subseteq B_{0}$ and as also $A=A_{0} \times B_{0}$, $=B$. If $A=A_{1} \times B$, then necessarily $A_{1} \subseteq B_{0}$ and as also $A=A_{0} \times B_{0}$, we conclude that $A_{1}=$ $B_{0}$. In the contrary case, $B \cap A_{1}=Z(K)$ has rank at most 2. Since $F$ acts irreducibly on $B=A_{0}$, the only possibility is that $m=2$ and $B=Z(K)$, whence $A=A_{1}$. However, $F$ centralizes $A / A_{0}=A / B$ and as $F$ acts on $K$ and $K / Z(K) \cong L_{3}(4),(2.21(\mathrm{vi}))$ implies that $F$ centralizes $K$, contrary to the fact that $F$ acts nontrivially on $A_{0}=Z(K)$. Hence this case is excluded and so we have proved

LEMMA 8.31. The following conditions hold:

(i) $A_{0}=B$ and $A_{1}=B_{0}^{\prime}$,

(ii) $Z(K)=1$ and $A=A_{1} \times B$.

\section{Lemma 8.32. $F$ centralizes $K$.}

Proof. By the preceding lemma, $F$ centralizes $A_{1}$. Since $F$ acts on $K$ and $|F|$ is odd, by (2.20) and (2.21) either $F$ centralizes $K$ or $K \cong U_{3}\left(2^{n}\right)$. Hence it suffices to consider the latter case. Then $T=O_{2}\left(C_{K}\left(A_{1}\right)\right)$ is a Sylow 2-subgroup of $K$ and $T$ is $F$-invariant. Since $x \in B_{0}=A_{1}, T$ centralizes $x$ 
and so $T \subseteq C_{G}(x)$, whence $T$ acts on $L_{x}$. Now $J$ is a component of $L_{x}$ and $A_{0}=B \subseteq J$. Since $T$ centralizes $A_{0}, T$ leaves $J$ invariant and so $T$ leaves $T_{0}=\bar{O}_{2}\left(C_{J}\left(A_{0}\right)\right)$ invariant. Since $T$ centralizes $A_{0}$, it follows from (2.20) that $T_{0} T=T_{0} C_{T_{0} T}(J)$. Thus $F$ centralizes $\left(T_{0} T\right) / T_{0}$. However, $T_{0}$ $\cap T=1$ as $\Omega_{1}\left(T_{0}\right)=A_{0}, \Omega_{1}(T)=B_{0}$, and $A_{0} \cap B_{0}=1$. Thus $T \cong T_{0} T / T_{0}$ and as $T$ is $F$-invariant, we conclude that $F$ centralizes $T$. But now we can apply (2.20) to obtain that $F$ centralizes $K$ in this case as well.

By the lemma, $K \subseteq C_{G}(F) \subseteq N_{G}(F)$. We next prove

Lemma 8.33. $K$ is not normal in $N_{G}(F)$.

Proof. Assume false, in which case $N_{J}(F)$ normalizes $K$. But $J=$ $\left\langle A_{0}, N_{J}(F)\right\rangle$ by $(2.36($ ii) $)$ and so $J$ normalizes $K$, contrary to our choice of $J$.

To complete the proof of Proposition 8.1, we shall now argue, to the contrary, that $K$ is normal in $N_{G}(F)$.

We first prove

Lemma 8.34. If $b \in B_{0}^{\#}$, then $J=L_{b}$. In particular, $C_{G}(b)$ normalizes $J$.

Proof. We have that $J$ is a component of $L_{x}$ for some $x$ in $B_{0}^{\#}$ and that $B_{0} \subseteq A \in M(G)$, so $J$ is a component of $L_{b}$ for any $b$ in $B_{0}^{\#}$ by (2.37). Suppose $L_{b}$ has a second component $L \neq J$. Since $A_{0}=B \subseteq J$ and $J$ centralizes $L$, it follows from Lemma 8.8 that $L$ normalizes $K$. We know that $L$ is of even type or of type $L_{2}(5)$ and that $L_{2}(5)$ is evenly embedded in $L(G)$. Hence $A \cap L \in M(L)$ by (2.37). Clearly then $A \cap L$ does not centralize $L$ and as $B$ centralizes $L$, we see that $A_{1}$ does not centralize $L$. Since $L / Z(L)$ is simple, this implies that $\left[A_{1}, L\right]=L$. But as $A_{1} \subseteq K$ and $L$ normalizes $K,\left[A_{1}, L\right] \subseteq K$ and so $L \subseteq K$. Therefore $L \subseteq L\left(C_{K}(b)\right)$. On the other hand, $b \in B_{0}=A_{1}$. Considering the possibilities for $K$ and noting that $Z(K)=1$, we conclude now from $(2.26)$ that $L\left(C_{K}(b)\right)=1$. This contradiction established the lemma.

We next prove

LEMMA 8.35. The following conditions hold:

(i) $S$ normalizes both $J$ and $K$;

(ii) $C_{S}(\langle K, J\rangle)=1$;

(iii) If $m(J) \leqslant 3$, then no element of $S$ induces a nontrivial outer automorphism of $J$.

Proof. Since $S \subseteq N_{G}(A), S$ normalizes $K$. Thus $S$ normalizes $A_{1}=$ $B_{0}$ and consequently $S$ centralizes some involution $b$ of $B_{0}$. But $J \triangleleft C_{G}(b)$ by the preceding lemma and so $S$ normalizes $J$ as well. Furthermore $J$ is a component of $L_{b}$. If $m(J) \leqslant 3$, then either $J \cong L_{2}(4), L_{2}(8), U_{3}(4), U_{3}(8)$, or 
$S z(8)$. Since $L_{2}(4) \cong L_{2}(5)$ is evenly embedded in $L(G)$, it follows from (2.18) that no element of $S$ induces a nontrivial outer automorphism of $J$. Finally setting $S_{0}=C_{S}(\langle K, J\rangle)$, we have that $S_{0} \triangleleft S$, so either $S_{0}=1$ or $S_{0}$ contains an involution $z$ of $Z(S)$. In the latter case, $z \in Z$ and so $z \in C_{A}(K)$ $\cap C_{A}(J)=B \cap B_{0}=1$, a contradiction. Thus $S_{0}=1$ and the lemma is proved.

We now set $C=C_{G}(F)$, so that $C \subset G$ as $F \neq 1$ and $K \subseteq C$ by Lemma 8.32. We have that $T=S \cap K \in S(K)$ and we let $U$ be a Sylow 2-subgroup of $C$ containing $T$.

\section{Lemma 8.36. $U$ normalizes $K$ and $C_{U}(K)=1$.}

Proof. Since $K$ is simple, the lemma holds if $U=T$, so assume $U \supset T$ and set $V=N_{U}(T)$, in which case also $V \supset T$. We have that $V$ normalizes $Z(T) \subseteq A_{1}=B_{0}$ and so $V$ centralizes an involution $b$ of $B_{0}$. But now Lemma 8.34 implies that $V$ normalizes $J$. However, $V$ centralizes $F$ and consequently $V$ centralizes $J$ by $(2.20(v i))$. But by the preceding lemma $C_{G}(K) \cap$ $C_{G}(J)$ has odd order and so $C_{V}(K)=1$. Since $V \supset T$ and $K$ is of even type, it follows now from (2.18) that $K \cong L_{2}\left(2^{r}\right)$ and $|V: T|=2$. Since $r \geqslant$ $3, T$ is the unique abelian subgroup of index 2 in $V$ (using $(2.20(\mathrm{v})$ )) and so $T$ is characteristic in $V$. Since $V=N_{U}(T)$, this forces $V=U$ and the lemma is proved.

Note that our argument actually yields that $T$ is of index at most 2 in $U$, with equality holding only if $K \cong L_{2}\left(2^{r}\right)$.

Finally we prove

\section{Lemma 8.37. We have $K=L(C)$.}

Proof. Set $\bar{C}=C / O(C) . \bar{K}$ acts faithfully on $L(\bar{C}) O_{2}(\bar{C})$ by (2.8). As $|\bar{U}| \leqslant 2|\bar{T}|$, it follows easily that $\bar{K} \subseteq L(\bar{C})$ and $L(\bar{C})$ is quasisimple. We have that $m(\bar{K})=r \geqslant 3$, that $\bar{K}$ is of even type, and that $\bar{T}$ is of index at most 2 in a Sylow 2-subgroup of $L(\bar{C})$. Considering the various elements of $\hat{A}$, we conclude at once from (2.25) that either $\bar{K}=L(\bar{C})$ or $\bar{K} \cong L_{2}(8)$ and $L(\bar{C})$ is of Ree type.

Consider the latter possibility, in which case $K \cong L_{2}(8)$ and $r=3$, and $m(J) \leqslant 3$ by our maximal choice of $K$. Hence by Lemma 8.35 no element of $S$ induces a nontrivial outer automorphism of $J$. Thus $S=C_{S}(J) \times T_{0}$, where $T_{0}=S \cap J \in S(J)$. But $C_{S}(J)$ acts faithfully on $K \cong L_{2}(8)$ and consequently $C_{S}(J)=A_{1}=T$. Hence $S=A_{1} \times T_{0}$ and as $T_{0}$ is a Suzuki 2group, it follows that $\Omega_{1}(S)=A_{1} \times \Omega_{1}\left(T_{0}\right)$ is abelian. But then obviously $\Omega_{1}(S)$ is strongly closed in $S$, contrary to Proposition 3.1. Therefore this case cannot arise and we conclude that $\bar{K}=L(\bar{C})$.

Clearly to complete the proof of the lemma, we need only show that $K$ centralizes $Y=O(C)$. But $A_{1}=B_{0}$ acts on $Y$ and so $Y=\left\langle C_{Y}(b) \mid b \in B_{0}^{\#}\right\rangle$, 
whence $Y$ normalizes $J$ by Lemma 8.34. Since $C=C_{G}(F)$ and $|F|$ is odd, we have that $F \subseteq Y$ and $Y$ centralizes $F$. It follows therefore from (2.20(vi)) that $Y$ normalizes $B$. By Lemma $8.8 Y$ normalizes $K$ whence $[Y, K] \subseteq$ $K \cap Y$ and so $[Y, K]$ is normal in $K$ of odd order, forcing $[Y, K]=1$. Thus $K$ centralizes $Y$ and the lemma is proved.

By the lemma, $K$ is characteristic in $C_{G}(F)$ and so $K$ is normal in $N_{G}(F)$, contrary to Lemma 8.33. This at last completes the proof of Proposition 8.1.

9. The normalizer of the even $A$-hull. At last we can attain the goal stated in $\S 4$.

Proposition 9.1. $L(G)$ is empty.

By our earlier results, we are reduced to the case in which $L_{1}(G)$ is empty or consists of an evenly embedded $L_{2}(5), O\left(C_{G}(x)\right)=1$ for every $x$ in $I(G)$, and $L_{x}=L\left(C_{G}(x)\right)$ is semisimple for every $x$ in $I(G)$. Moreover, under the assumption that $L(G)$ is nonempty, we have shown in Proposition 8.1 that for some $A$ in $M(G)$, the even $A$-hull

$$
X_{A}=\left\langle L_{x} \mid x \in A^{\#}\right\rangle
$$

is a nontrivial semisimple subgroup of $G$ with the following properties:

(a) $X_{A}$ has a component $K$ such that $r=m(K / Z(K))$ is maximal among the elements of $L(G)$;

(b) The components of $X_{A}$ are elements of $L(G ; A)$;

(c) Every element of $L(G ; A)$ either lies in $K$ or centralizes $K$.

We set $M=N_{G}\left(X_{A}\right)$. As in $\S 7$, we shall derive a contradiction by showing that $M$ is strongly embedded in $G$, in which case (2.60) will imply that $G$ is a simple Bender group contrary to our choice of $G$ as a maximal counterexample to the Main Theorem.

We let $K=K_{1}, K_{2}, \cdots, K_{m}$ be the components of $X_{A}$ for which $m\left(K_{i} / Z\left(K_{i}\right)\right)=r, 1 \leqslant i \leqslant m$, and denote their product by $Y$. Then $Y$ is a characteristic semisimple subgroup of $X_{A}$. Since $N_{G}(A)$ obviously normalizes $X_{A}$, this yields

Lemma 9.2. We have $N_{G}(A) \subseteq N_{M}(Y)$.

In particular, if $S$ is any Sylow 2- subgroup of $G$ containing $A$, it follows that $S \subseteq N_{M}(Y)$.

We also have

Lemma 9.3. If $L \in L(G ; A)$ and $m(L / Z(L))=r$, then $L=K_{i}$ for some $i, \quad 1 \leqslant i \leqslant m$. 
Proof. Since $A \in M(G)$ and $K$ is a component of $X_{A}$, (2.37), applied to the group $X_{A} A$, implies that $K$ is $A$-invariant and so $K$ contains an element $x$ of $A^{\#}$. Thus $x$ centralizes any component $F \neq K$ of $X_{A}$. By (b), $F$ is a component of $L_{b}$ for some $b$ in $A^{\#}$. Hence by $L$-balance, $F \subseteq L_{x}$. Thus $X_{A}$ $\subseteq K L_{x} \subseteq X_{A}$. Now (c) yields that either $L \subseteq K$ or $L \subseteq L_{x}$.

Consider the latter case. We have that $L$ is a component of $L_{a}$ for some $a$ in $A^{\#}$. Then $L$ centralizes $x$ and as $\langle x, a\rangle \subseteq A \in M(G),(2.37)$ yields that $L$ lies in a unique component $J$ of $L_{x}$. Thus $L \subseteq L\left(C_{J}(a)\right)$. On the other hand, in the former case, $L \subseteq L\left(C_{K}(a)\right)$.

Since the elements of $L(G)$ are either of even type or isomorphic to $L_{2}(5)$ and since $r=m(L / Z(L))=m(K / Z(K)) \geqslant m(J / Z(J))$, it follows now from (2.27) that we have $L=K$ or $J$, as the case may be. In either case we conclude that $L$ is a component of $X_{A}$ and the lemma follows.

We next prove

LEMMA 9.4. We have $O(M)=1$.

Proof. Setting $D=O(M)$, it will clearly suffice to prove that $D_{a}=$ $C_{D}(a)=1$ for $a$ in $A^{\#}$. But as $L_{a} \subseteq C_{M}(a), L_{a}$ normalizes $D_{a}$. Since $O\left(L_{a}\right) \subseteq O\left(C_{G}(a)\right)=1$ and $L_{a} \triangleleft C_{G}(a)$, it follows that $\left[L_{a}, D_{a}\right] \subseteq L_{a} \cap D_{a}=$ 1 , so $\bar{D}_{a}$ centralizes $L_{a}$. Furthermore, if $Q=O_{2}\left(C_{G}(a)\right)$, then $Q$ is $A$-invariant, so $Q A$ is a 2-group and consequently $Q$ normalizes $A$. Thus $Q \subseteq M$ and so $\left[Q, D_{a}\right] \subseteq Q \cap O(M)=1$. Hence $D_{a}$ centralizes $Q$ as well as $L_{a}$. But now (2.8) implies that $D_{a}=1$.

LEMmA 9.5. If $a \in A^{\#}$, then $L_{a}=L\left(C_{L(M)}(a)\right)$.

Proof. Set $J=L\left(C_{L(M)}(a)\right)$. Since $L_{a} \subseteq M, L_{a} \subseteq L\left(C_{M}(a)\right)$ and as $O(M)=1,(2.41)$ implies that $L_{a} \subseteq L(M)$. It follows at once now that $L_{a} \triangleleft J$. On the other hand, $Q=O_{2}\left(C_{G}(a)\right) \subseteq M$ as in the preceding lemma. Thus $Q$ normalizes $L(M)$ and so normalizes $J$. But $J$ normalizes $Q$ as $J \subseteq C_{G}(a)$ and consequently $[J, Q] \subseteq J \cap Q \subseteq Z(J)$. The three subgroup lemma now forces $Q$ to centralize $J$.

Finally as $L_{a} \triangleleft J$, either equality holds or $J$ contains a component $J_{0}$ which centralizes $L_{a}$. However, as $J_{0}$ also centralizes $Q$, this contradicts (2.8). Thus $L_{a}=J$, as asserted.

We now choose $S$ in $S(G)$ with $A \subseteq S$ and we set $Z=Z(S)$. We know that $S \subseteq N_{M}(Y)$. We next prove

LEMMA 9.6. Every component of $L(M)$ centralizes an involution of $Z$.

Proof. $S$ permutes the components of $L(M)$. If there are at least two 
$S$-orbits, we can write $L(M)=L_{1} L_{2}$, where $L_{1}, L_{2}$ are each a nontrivial $S$-invariant product of components of $L(M)$. Then $Z \cap L_{i} \neq 1$ and $Z \cap L_{i}$ centralizes $L_{j}, i \neq j, 1 \leqslant i, j \leqslant 2$. Clearly the lemma holds in this case. Hence we can suppose that $S$ acts transitively on $L(M)$, which implies that $L(M)=Y$. If $Y=K$, then $K$ is $S$-invariant and $C_{S}(K) \neq 1$ as $K$ centralizes some $a$ in $A^{\#}$. But $C_{S}(K) \triangleleft S$ and so $C_{Z}(K) \neq 1$; again the lemma holds. On the other hand, by (2.13), every $S$-orbit consists of at most two components, so the only other possibility is that $Y=K_{1} K_{2}$ and $K_{1}, K_{2}$ have abelian Sylow 2-subgroups. If $C_{S}(Y) \neq 1$, then $C_{Z}(Y) \neq 1$ and again the lemma follows.

Assume finally that $C_{S}(Y)=1$, so that $S$ acts faithfully on $Y$ and $S=$ $T\langle x\rangle$, where $T$ is the subgroup of $S$ leaving $K_{1}, K_{2}$ invariant and $x$ interchanges $K_{1}, K_{2}$. Furthermore, $K=K_{1} \cong L_{2}\left(2^{r}\right)$ as $K$ has abelian Sylow 2 subgroups and $K$ is of even type or isomorphic to $L_{2}(5)$. If some element of $T$ induces a nontrivial outer automorphism of $K$, then $S^{\prime} \cap K \neq 1$. But $S^{\prime} \cap K$ $\subseteq Z(S)=Z$ and $x$ centralizes $Z$, so $x$ leaves $K$ invariant, a contradiction. Hence no such element exists and this implies that $T=S \cap Y$. Hence if $T_{i}=S \cap K_{i}$, $i=1,2$, we have that $S=\left(T_{1} \times T_{2}\right)\langle x\rangle$ with $x$ interchanging $T_{1}$ and $T_{2}$. But now as in Lemma 8.14 we can choose $x$ to be an involution and consequently $S=$ $E_{2} r \sim E_{2}$, contrary to (2.74).

Recall that we have defined the even $Q$-hull $X_{Q}=\left\langle L_{x} \mid x \in I(Q)\right\rangle$ for any subgroup $Q$ of $G$. We can now prove

LEMma 9.7. We have $X_{Z}=X_{A}=L(M)$.

Proof. Since $Z \subseteq A$ and $X_{A} \triangleleft M$, we have $X_{Z} \subseteq X_{A} \triangleleft L(M)$. Since $A \cap X_{A} \neq 1$, any component $M_{0}$ of $M$ not in $X_{A}$ must centralize some $a$ in $A^{\#}$, whence $M_{0} \subseteq L_{a} \subseteq X_{A}$ by Lemma 9.5 and the definition of $X_{A}$, a contradiction. Thus $X_{A}=L(M)$. Now Lemmas 9.5 and 9.6 and $L$ balance imply that $X_{A} \subseteq X_{Z}$, so $X_{A}=X_{Z}$ and the lemma holds.

The next lemma is crucial.

Lemma 9.8, We have $X_{S}=X_{A}$.

Proof. We must show that $L_{x} \subseteq X_{A}$ for any $x$ in $I(S)$. Fix such an $x$, set $B=C_{A}(x)$ and let $J$ be an arbitrary component of $L_{x}$. We need only prove that $J \subseteq X_{A}$. We have that $B \subseteq C_{G}(x)$ and so $B$ acts on $L_{x}$.

Suppose first that either

(a) $m(B) \geqslant r+1$ and $J \not L_{2}$ (9) or

(b) $m(B) \geqslant 4$ and $J \cong L_{2}(9)$.

If $B$ normalizes $J$, then by (2.23), $J$ centralizes some $b$ in $B^{\#}$. But then by 
$L$-balance, $J \subseteq L_{b}$ and as $b \in A$, it follows that $J \subseteq X_{A}$. On the other hand, if $B$ does not leave $J$ invariant, then by (2.12) and (2.13), the normal closure $V$ of $J$ under the action of $B$ consists of two components of $L_{x}$ and $V=$ $\left\langle L\left(C_{V}(b)\right) \mid b \in B^{\#}\right\rangle$. Again $L$-balance and $B \subseteq A$ yield that $V \subseteq X_{A}$. Since $J$ is one of the components of $V$, we conclude in this case as well that $J \subseteq X_{A}$. Hence we can suppose that neither (a) nor (b) hold.

Assume next that $x$ does not leave $K$ invariant, in which case $L=$ $L\left(C_{K K^{x}}(x)\right)$ is isomorphic to a homomorphic image of $K$ by (2.12). The proof of Lemma 9.6 shows, if $C_{S}\left(K K^{x}\right)=1$, that $S=E_{2^{r}} \sim E_{2}$, contrary to (2.74). Hence $C_{S}\left(K K^{x}\right) \neq 1$ and it follows that $K K^{x}$ centralizes some $z$ in $Z^{\#}$. By Lemma 9.5 and our maximal choice of $K, K$ and $K^{x}$ are then components of $L_{z}$. But then (2.43) implies that the normal closure $W$ of $L$ in $L_{x}$ consists of one or two components and $L=L\left(C_{W}(z)\right)$. We shall argue that $W \subseteq$ $X_{A}$ and that $A \cap W \neq 1$. Once this is proved, it will follow that $J \subseteq X_{A}$. Indeed, either $J \subseteq W \subseteq X_{A}$ or $J$ centralizes $W$, whence $J$ centralizes $a \in$ $(W \cap A)^{\#}$ and again $J \subseteq L_{a} \subseteq X_{A}$ by $L$-balance.

Since $z \in Z$, we have that $z \in B$. Also as $m(A) \geqslant 5$, we have that $m(B)$ $\geqslant 3$. If $W$ consists of two components, then (2.12) and (2.13) imply that $W$ is $B$-invariant and that $W=\left\langle L\left(C_{W}(b)\right) \mid b \in B^{\#}\right\rangle$. Again $L$-balance applies to give $W \subseteq X_{A}$. On the other hand, if $W$ consists of a single component, then either $W=L \subseteq L_{z} \subseteq X_{A}$ or $z$ induces a nontrivial outer automorphism of $W$. Since $L\left(C_{W}(z)\right) \neq 1$, the only possibility is that $W \cong L_{2}\left(2^{2 n}\right)$ and $L \cong L_{2}\left(2^{n}\right)$ for some $n \geqslant 2$. But as $L$ is isomorphic to a homomorphic image of $K$, we must have $n=r$, whence $m(W)>r$, contrary to our maximal choice of $K$. We conclude that $W \subseteq X_{A}$. Furthermore, as $A \cap K \in M(K)$ and $x$ normalizes $A \cap K K^{x}$, it is immediate that $A \cap L \neq 1$. Since $L \subseteq W$, it follows that $A \cap$ $W \neq 1$, so both our assertions are proved. Thus we can also assume without loss that $x$ leaves $K$ invariant.

Now consider the case in which for some $u$ in $K, y=x^{u}$ centralizes $A_{1}=A \cap K$ and lies in $S$. We know that $K$ centralizes $z$ in $Z^{\#}$ and it follows now from the definition of $r$ that $A_{1}\langle z\rangle$ has rank at least $r+1$. Since $B_{1}=C_{A}(y) \supseteq A_{1}\langle z\rangle$, we have that $m\left(B_{1}\right) \geqslant r+1$. Furthermore, $J^{u}$ is a component of $L_{y}$. We argue next that also $m\left(B_{1}\right) \geqslant 4$. Clearly this is the case if $r \geqslant 3$, so assume $r=2$, in which case $K \cong L_{2}(5), U_{3}(4)$, or $L_{2}(9)$. As $y \in S, y$ normalizes $A$; and as $y=x^{u}, y$ normalizes $K$. Thus $y$ normalizes $A_{2}=C_{A}(K)$. If $m\left(A_{2}\right) \geqslant 3$, then $m\left(C_{A_{2}}(y)\right) \geqslant 2$; and since $A_{1} \times C_{A_{2}}(y) \subseteq$ $B_{1}$, we conclude $m\left(B_{1}\right) \geqslant 4$. Thus we can also assume $m\left(A_{2}\right) \leqslant 2$, which is possible only if $K \cong L_{2}(9)$. In this case we apply (2.22) to conclude that $K_{0}=$ $N_{K}\left(A_{1}\right) \cong S_{4}$ and that $A=A_{1} \times A_{0}$, where $A_{0}=C_{A}\left(K_{0}\right)$. Since $y$ leaves 
both $K_{0}$ and $A$ invariant, it leaves $A_{0}$ invariant and as $m(A) \geqslant 5$, we have $m\left(A_{0}\right) \geqslant 3$. As in the case $m\left(A_{2}\right) \geqslant 3$, this yields that $m\left(B_{1}\right) \geqslant 4$ in this case as well.

We see then that condition (a) or (b) above holds with $y, B_{1}$, and $J^{u}$ in the roles of $x, B$, and $J$, respectively. We can therefore apply the first portion of the proof to conclude that $J^{u} \subseteq X_{A}$. Since $u \in K \subseteq X_{A}$, it follows in this case as well that $J \subseteq X_{A}$.

We argue next that such an element $y=x^{u}$ exists if either $x$ induces an inner automorphism of $K$ or if $K \cong L_{2}(9)$. Indeed set $H=K\langle x\rangle, C=$ $C_{H}(K)$ and $\bar{H}=H / C$. Clearly $S \cap H \in S(H)$ and so $C \subseteq S$. We have that $A_{1}=A \cap K \in M(K)$ and using (2.16) we obtain that $\bar{A}_{1} \in M(\bar{K})$. If $x$ induces an inner automorphism of $K$, then $\bar{H}=\bar{K}$ and it follows from (2.31) that $\bar{y}=$ $\bar{x}^{\bar{u}} \in \bar{A}_{1}$ for some $\bar{u}$ in $\bar{K}$. But then $y=x^{u} \in A_{1} C$ for some $u$ in $K$. Thus $y \in$ $S$ and $y$ centralizes $A_{1}$. On the other hand if $K \cong L_{2}(9)$ and $x$ induces a nontrivial outer automorphism of $K$, then $\overline{S \cap H}=(\overline{S \cap K}) \times\langle\bar{w}\rangle \cong D_{8} \times E_{2}$ for some $\bar{w} \in I\left(\overline{S \cap H)}\right.$ and $\bar{x}=\bar{w} \bar{a}$ for some $\bar{a}$ in $I(\overline{S \cap K})$. But $\bar{A}_{1}\langle\bar{w}\rangle$ contains representatives of each conjugacy class of involutions of $\bar{H}$ and so there exists $u \in$ $K$ such that $y=x^{u} \in S$ and $\bar{y} \in \overline{A_{1}}\langle\bar{w}\rangle$. Thus $y$ centralizes $A_{1}$ in this case as well.

We are thus reduced to showing that $J \subseteq X_{A}$ in the case that $x$ induces a nontrivial outer automorphism of $K$ and $K \nRightarrow L_{2}(9)$. By (2.18), we have $K \cong$ $L_{2}\left(2^{r}\right)$. Again $z$ centralizes $K$ for some $z$ in $Z^{\#}$. Then $K$ is a component of $L_{z}$ and $x \in C_{G}(z)$. Since $L_{1}(G)$ is either empty or consists of an evenly embedded $L_{2}(5)$, we cannot have $r=2$. Thus $r \geqslant 4$ and $L=L\left(C_{K}(x)\right)$ $\neq 1$. Again by (2.43) the normal closure $W$ of $L$ in $L_{x}$ consists of one or two components and $L=L\left(C_{W}(x)\right)$. As in the case that $x$ does not leave $K$ invariant, we need only prove that $W \subseteq X_{A}$ and $A \cap W \neq 1$ to reach the desired conclusion $J \subseteq X_{A}$.

We have that $A_{1}=A \cap K \cong E_{2^{r}}$ and that $B_{0}=C_{A_{1}}(x) \cong E_{2^{r / 2}}$. In particular, $B_{0} \subseteq L \subseteq W$ and so $A \cap W \neq 1$. Furthermore, $B_{0} \times\langle z\rangle \subseteq B$. If $W$ consists of two components, then, as usual, (2.12) and $L$-balance imply that $W \subseteq$ $X_{A}$. In the contrary case, we apply (2.36(iv)) to obtain likewise that $W=$ $\left\langle L\left(C_{W}(b)\right) \mid b \in B^{\#}\right\rangle$ and again $L$-balance implies that $W \subseteq X_{A}$. Thus $W$ has the required properties and so $J \subseteq X_{A}$ in this final case.

As a corollary, we have

LEMMA 9.9. If $R$ is a subgroup of $S$ containing $Z$, then

$$
N_{G}(R) \subseteq M \text {. }
$$

Proof. We have $X_{Z} \subseteq X_{R} \subseteq X_{S}$. However, $X_{Z}=X_{A}=X_{S}$ by Lemmas 
9.7 and 9.8 and we conclude that $X_{A}=X_{R}$. But by definition of $X_{R}$, clearly $N_{G}(R)$ normalizes $X_{R}$. Since $X_{R}=X_{A}$ and $M=N_{G}\left(X_{A}\right)$, the desired conclusion $N_{G}(R) \subseteq M$ now follows.

This in turn yields

Lemma 9.10. $M$ controls fusion in $S$.

Proof. We need only prove that $N_{G}(D) \subseteq M$ for each $D$ in the Goldschmidt conjugation family $D$ of (2.70). But $\bar{Z} \subseteq C_{S}(D) \subseteq D \subseteq S$ for each $D$ in $D$ and so the desired inclusions follow from the preceding lemma.

Now we can obtain our objective.

LeMMA 9.11. $M$ is strongly embedded in $G$.

Proof. We have $N_{G}(S)$ normalizes $X_{S}=X_{A}$ and so $N_{G}(S) \subseteq M$. Hence it will suffice to show that $H=C_{G}(x) \subseteq M$ for each $x$ in $I(S)$. By the preceding lemma, we need only treat the case in which $x$ is extremal in $S$. Hence if $P=C_{S}(x)$, we can assume that $P$ is a Sylow 2-subgroup of $H$. Let $F$ denote the inverse image of $O_{2}\left(H / L_{x}\right)$ in $H$. Then $F \triangleleft H$ and $F=L_{x} R$, where $R=P \cap F$ is a Sylow 2-subgroup of $F$. By (2.52), we have that $Z(P) \subseteq$ $R$. Since $Z \subseteq P \subseteq S$, it follows that $Z \subseteq Z(P)$ and hence, in particular, that $Z \subseteq R$.

By the Frattini argument, $H=L_{x} N_{H}(R)$. Lemma 9.8 implies that $L_{x} \subseteq$ $X_{S}=X_{A} \subseteq M$, while Lemma 9.9 yields that $N_{G}(R) \subseteq M$ as $Z \subseteq R \subseteq S$. Thus $H \subseteq M$ and the lemma is proved.

As pointed out at the beginning of the section, Lemma 9.1 leads at once to a contradiction and so Proposition 9.1 is proved.

\section{REFERENCES}

1. J. Alperin, R. Brauer and D. Gorenstein, Finite simple groups of 2-rank 2, Scripta Math. 29 (1974), 191-214.

2. M. Aschbacher, Finite groups with a proper 2-generated core, Trans. Amer. Math. Soc. 197 (1974), 87-112.

3. H. Bender, Transitive Gruppen gerader Ordnung, in denen jede Involution genau einen Punkt festlässt, J. Algebra 17 (1971), 527-554. MR 44 \#5370.

4. M. J. Collins, The characterisation of the Suzuki groups by their Sylow 2-subgroups, Math. Z. 123 (1971), 32-48. MR 46 \#7366.

5. P. Fong, Sylow 2-groups of small order (unpublished).

6. G. Glauberman, Central elements in core-free groups, J. Algebra 4 (1966), 403-420. MR 34 \#2681.

7. D. Goldschmidt, Sylow 2-subgroups with non-elementary centers. Representation theory of finite groups and related topics, Proc. Sympos. Pure Math., vol. 21, Amer. Math. Soc., Providence, R. I., 1971, pp. 53-56.

8. - A conjugation family for finite groups, J. Algebra 16 (1970), 138-142. MR 41 \#5489.

9. - Solvable signalizer functors on finite groups, J. Algebra 21 (1972),

137-148. MR 45 \#6913. 
$321-340$

10. D. Goldschmidt, 2-signalizer functors on finite groups, J. Algebra 21 (1972),

11. 2-fusion in finite groups, Ann. of Math. (2) 99 (1974), 70-117.

12. D. Gorenstein, Finite groups, Harper \& Row, New York, 1968. MR 38 \#229.

13. - On finite simple groups of characteristic 2 type, Inst. Hautes Études Sci.

Publ. Math. No. 36 (1969), 5-13. MR 41 \#5484.

14. - On the centralizers of involutions in finite groups, J. Algebra 11 (1969), 243-277. MR 39 \#1540.

15. - Centralizers of involutions, Finite Simple Groups, M. B. Powell and G. Higman (editors), Academic Press, New York, 1971.

16. D. Gorenstein and K. Harada, Finite groups whose Sylow 2-subgroups are the direct product of two dihedral groups, Ann. of Math. (2) 95 (1972), 1-54. MR 47 \#1939.

17. D. Gorenstein and M. Harris, Finite groups with product fusion, Ann. of Math. (to appear).

18. D. Gorenstein and J. Walter, The $\pi$-layer of a finite group, Illinois $\mathrm{J}$. Math. 15 (1971), 555-564. MR 44 \#6812.

19. - Centralizers of involutions in balanced groups, J. Algebra 20 (1972), 284-319. MR 45 \#2008.

20. - Balance and generation in finite groups, J. Algebra 33 (1975), 224-287.

21. R. L. Griess, Jr., Schur multipliers of known finite simple groups, Proc. Amer. Math. Soc. 78 (1972), 69-71. MR 44 \#6823.

22. J. G. Thompson, Nonsolvable finite groups all of whose local subgroups are solvaable, Bull. Amer. Math. Soc. 74 (1968), 383-437. MR 37 \#6367.

23. J. H. Walter, Finite groups with abelian Sylow 2-subgroups of order 8, Invent. Math. 2 (1967), 332-376. MR 36 \#1531.

24. - The characterization of finite groups with abelian Sylow 2-subgroups, Ann. of Math. (2) 89 (1969), 405-514. MR 40 \#2749.

DEPARTMENT OF MATHEMATICS, STEVENS INSTITUTE OF TECHNOLOGY, HOBOKEN, NEW JERSEY 07030

\footnotetext{
DEPARTMENT OF MATHEMATICS, RUTGERS UNIVERSITY, NEW BRUNSWICK, NEW JERSEY 08903
} 